quatrième série-tome 42 fascicule 5 septembre-octobre 2009

$$
\begin{aligned}
& \text { ANNALES } \\
& \text { SCIENTIFIQUES } \\
& \text { de } \\
& \text { L'ECOLE } \\
& \text { NORMALE } \\
& \text { SUPÉRIEURE }
\end{aligned}
$$

Grégory MIERMONT

Tessellations of random maps of arbitrary genus 
Ann. Scient. Éc. Norm. Sup.

$4^{\text {e }}$ série, t. 42, 2009, p. 725 à 781

\title{
TESSELLATIONS OF RANDOM MAPS OF ARBITRARY GENUS
}

\author{
BY GRÉGORY MIERMONT
}

\begin{abstract}
We investigate Voronoi-like tessellations of bipartite quadrangulations on surfaces of arbitrary genus, by using a natural generalization of a bijection of Marcus and Schaeffer allowing one to encode such structures by labeled maps with a fixed number of faces. We investigate the scaling limits of the latter. Applications include asymptotic enumeration results for quadrangulations, and typical metric properties of randomly sampled quadrangulations. In particular, we show that scaling limits of these random quadrangulations are such that almost every pair of points is linked by a unique geodesic.

RÉsumé. - Nous examinons les propriétés de mosaïques de type Voronoï sur des quadrangulations bipartites de genre arbitraire. Ceci est rendu possible par une généralisation naturelle d'une bijection de Marcus et Schaeffer, permettant de décrire ces mosaïques par des cartes étiquetées avec un nombre fixé de faces, dont nous déterminons les limites d'échelle. Parmi les applications de ces résultats, figurent le comptage asymptotique des quadrangulations, ainsi que des propriétés métriques typiques de quadrangulations choisies au hasard. En particulier, nous montrons que les limites d'échelles de ces quadrangulations aléatoires sont telles que presque toute paire de points est liée par un unique chemin géodésique.
\end{abstract}

\section{Introduction}

\subsection{Motivation}

A map is a graph embedded into a compact orientable surface without boundary, yielding a cell decomposition of the surface, and considered up to orientation-preserving homeomorphisms. Random maps are considered in the physics literature on quantum gravity as discretized versions of random surfaces [2]. This approach allows one to perform computations of certain integrals with respect to an ill-defined measure on surfaces, by approximating them by finite sums over maps. From a mathematical perspective, this leads to the stimulating problem of existence of a measure on compact surfaces arising as the scaling limit of, say,

This research was supported by the Fondation des Sciences Mathématiques de Paris and by the ANR grant ANR-08-BLAN-0190. 
uniform random triangulations of the sphere (or the compact orientable surface of genus $g$ ) with a large number of faces.

Important progress has been made in this direction in the recent years, thanks to bijective approaches initiated in Schaeffer's thesis [42]. They allowed Chassaing \& Schaeffer [11] to obtain the scaling limits for the radius and profile of a uniform rooted planar quadrangulation with $n$ faces, considered as a metric space by endowing its vertices with the usual graph distance. In particular, they showed that typical graph distances between vertices of such random quadrangulations are of order $n^{1 / 4}$. Generalizations of this result have been obtained for much more general families of random maps by Marckert, Weill and the author in $[30,34,36,45]$, relying on generalizations of Schaeffer's bijection by Bouttier, Di Francesco \& Guitter [7].

An important step has been accomplished by Le Gall [26], who showed that scaling limits of these random quadrangulations, considered in the Gromov-Hausdorff sense [22], must be homeomorphic to a metric quotient of the so-called Brownian Continuum Random Tree of Aldous [1]. This random quotient was introduced under the name of Brownian map by Marckert \& Mokkadem [32], who proved convergence of random quadrangulations towards this limit in a sense yet different from convergence in distribution in the GromovHausdorff topology. Subsequently, Le Gall \& Paulin [28] inferred that the Brownian map is homeomorphic to the two-dimensional sphere. At the present stage, it is however not known whether the scaling limit is uniquely determined as the Brownian map, which would lead to a satisfactory answer to the above mentioned problem.

A natural idea is to characterize the limit through its "finite-dimensional marginal distributions". From the point of view of metric measure spaces, this would mean to characterize the laws of mutual distances between an arbitrary number of randomly sampled points. This, however, seems hard to obtain. The approach of the present work, which deals with combinatorial, probabilistic and geometric aspects of maps, was initially motivated by another notion of finite-dimensional marginals, which takes into account the planar structure of the graphs that are considered, and roughly consists in studying the Voronoi tessellation on the map with sources taken at random.

Our central combinatorial result is a natural generalization of Schaeffer's bijective construction, to be introduced in Section 2. We do not restrict ourselves to the planar case, as the most natural framework is to consider maps of arbitrary (but fixed) genus $g$, so our bijection is really a generalized version of the Marcus-Schaeffer bijection in arbitrary genus [10, 33]. Let us explain briefly the idea of our construction. Recall that the Marcus-Schaeffer bijection encodes a genus- $g$ bipartite quadrangulation with a distinguished vertex by a labeled map of genus $g$ with one face, in such a way that the labels keep track of graph distances to the distinguished vertex in the quadrangulation. Our construction encodes a genus- $g$ bipartite quadrangulation with $k$ distinguished vertices by a labeled map of genus $g$ with $k$ faces (Theorem 4 and Corollary 1). In some sense, the faces of the encoding map correspond to the Voronoi tiles in the quadrangulation, with sources at the $k$ distinguished vertices, and the labels allow to keep track of graph distance of a vertex to the source of the Voronoi tile it belongs to. An important fact is that there is some flexibility in the construction, allowing one to consider a generalized Voronoi tessellation in which the distances to the $k$ sources are measured after addition of a delay depending on the source. 
The resulting set of labeled maps with a fixed number of faces is combinatorially much simpler than that of bipartite quadrangulations, and we are able to study their scaling limits. This is done in Section 4 (Theorem 5 and Propositions 3, 4), after showing how to encode labeled maps by appropriate processes in Section 3. Sections 5 and 7 give three applications of these scaling limit results for labeled maps, to combinatorial and geometric properties of bipartite quadrangulations.

The first application is a new derivation of known asymptotic enumeration results (Theorem 1), to which the short Section 5 is devoted. These are initially due to Bender \& Canfield [4], who obtained the asymptotic number of rooted maps of genus $g$ with $n$ edges ${ }^{(1)}$ by recursive decomposition methods, of a very different nature from our bijective study. These results have also been obtained in the recent work of Chapuy, Marcus \& Schaeffer [10], who completed the exact enumeration of maps of genus $g$ that was initiated in [33]. The starting point of our study (using the Marcus-Schaeffer bijection, which corresponds to the case $k=1$ of our bijection) is the same as in [10], but the rest of our approach is different, the arguments of Section 4 being of a more probabilistic nature.

Our other results concern the metric structure of randomly sampled bipartite quadrangulations. Let us indicate briefly what we mean by "random" in this paper. Most of the articles on the topic have focused on scaling limits as $n \rightarrow \infty$ of random families of maps conditioned to have $n$ faces or $n$ vertices. We prefer to randomize the number of faces, by using natural $\sigma$-finite measures on bipartite quadrangulations of fixed genus $g$, called the Boltzmann measures $\mathrm{Q}_{g}$ (the term being inspired from [3], see also [30, 34]), and which are obtained by assigning an appropriate weight to the faces. In the physics terminology, these measures correspond to the so-called grand-canonical measures, while the measures with fixed number of faces are the microcanonical measures. These measures are used to define natural "Boltzmann-Gibbs" distributions on quadrangulations, depending on an inverse temperature parameter $\beta$ that allows one to tune the average size of the quadrangulation and take scaling limits.

The existence of these scaling limits, considered with respect to the Gromov-HausdorffProkhorov topology on metric measure spaces, and stated in Theorem 2, is then obtained from the study of Section 4. This generalizes to arbitrary genera the fact that planar quadrangulations admit scaling limits for the Gromov-Hausdorff topology, as shown in Le Gall [26]. The theory of metrics on weighted metric spaces that is needed here is developed in Section 6.

The main result of the present paper, Theorem 3, gives qualitative information on the metric structure of the scaling limits of random bipartite quadrangulations. We show that these scaling limits are geodesic weighted metric spaces, in which two typical points are linked by a unique geodesic. The idea is to use our bijection for quadrangulations with $k=2$ marked vertices, in order to show that, if $x, y$ are typical points in the limiting random metric space $(X, d)$ arising as a scaling limit of random bipartite quadrangulations, the intersection of a geodesic between $x$ and $y$ and a geometric locus of the form

$$
\{z \in X: d(x, z)-d(y, z)=D\}, \quad D \in[-d(x, y), d(x, y)]
$$

(1) This is equivalent to our result, since such maps are in one-to-one correspondence with bipartite quadrangulations of same genus with $n$ faces [33, Proposition 1]. 
is a.s. reduced to a single point.

Theorem 3 is strongly reminiscent of the combinatorial results of the recent paper by Bouttier \& Guitter [8]. They show that in the planar case $g=0$, two typical vertices in a random quadrangulation with $n$ faces cannot be linked by two geodesics which are "very far" in the scale $n^{1 / 4}$, as $n \rightarrow \infty$. Our result confirms that geodesics between typical points become unique in the scaling limit, answering one of the questions raised in Section 5 of [8], though in our slightly different setting of Boltzmann-distributed maps. We also mention the recent work by Le Gall [27] on similar topics as the present paper and [8], and providing results on the exceptional geodesics as well.

In this work, the cardinality of a set $A$ is denoted by $|A|$.

\subsection{Embedded graphs and maps}

Let us introduce some formalism for embedded graphs and maps, which we partly borrow from [29].

Graphs on surfaces. Let $S$ be a compact connected orientable two-dimensional surface without boundary. It is well-known that such surfaces are characterized, up to homeomorphism, by an integer $g \geq 0$, called the genus of $S$, and that the topology of $S$ is that of the connected sum $\mathbb{S}_{g}$ of $g$ tori. The surface of genus 0 is the two-dimensional sphere.

A half-edge, or oriented edge $e$ in $S$ is a continuous path $c:[0,1] \rightarrow S$, considered up to continuous increasing reparametrization, which is either injective on $[0,1]$, or is injective on $[0,1)$ and satisfies $c(0)=c(1)$. In the latter case, $e$ is called a loop. The order on $[0,1]$ induces a natural orientation of the half-edge $e$, and its start and end points are defined as $e^{-}=c(0)$ and $e^{+}=c(1)$, a definition that depends only on the half-edge $e$ and not on the function $c$. Similarly, the image of $e$ is defined as $\operatorname{Im}(e)=c([0,1])$, and the interior of $e$ is $c((0,1))$. The reversal $\bar{e}$ of the half-edge $e$ is defined as the reparametrization class of $c(1-\cdot)$, so that $\bar{e}^{+}=e^{-}, \bar{e}^{-}=e^{+}$. An $e d g e$ is a pair of the form $\mathbf{e}=\{e, \bar{e}\}$ where $e$ is a half-edge.

An embedded graph in $S$, or simply a graph on $S$, is a pair $(V, \mathbf{E})$, where

- $\mathbf{E}$ is a non-empty, finite set of edges such that two distinct edges intersect, if at all, only at some of their endpoints

$-V$ is the set of vertices, i.e. of end-points of elements of $\mathbf{E}$.

We let $E$ be the set of half-edges corresponding to $\mathbf{E}$. The set $E$ has even cardinality and comes with the involution $e \mapsto \bar{e}$. An orientation of the edges is a choice of one half-edge inside each edge to form a set $E_{1 / 2} \subset E$ with same cardinality as $\mathbf{E}$.

A graph on $S$ determines faces, which are the connected components of the complement of $\bigcup_{e \in E} \operatorname{Im}(e)$ in $S$. Let $F$ be the set of faces of the graph.

Maps. We say that the embedded graph $(V, \mathbf{E})$ is a map if all faces are simply connected (hence determining a cell complex structure on $S$ ). A map is necessarily a connected graph by [29, Lemma 1.5].

The degree of a face in a map is the number of edges it is incident to, where it is understood that edges that are incident twice to the same face should be counted twice. By Euler's formula,

$$
|V|-|\mathbf{E}|+|F|=\chi(g)
$$

$4^{\mathrm{e}}$ SÉRIE - TOME $42-2009-\mathrm{N}^{\circ} 5$ 
where $\chi(g)=2-2 g$ is the Euler characteristic of $S$.

A map is rooted if it comes with a distinguished half-edge $e_{*} \in E$.

We declare two (rooted) maps $\mathbf{m}, \mathbf{m}^{\prime}$ on surfaces $S, S^{\prime}$ to be isomorphic if there exists a homeomorphism $S \rightarrow S^{\prime}$ that preserves the orientation, and sends the vertices and edges of $\mathbf{m}$ to those of $\mathbf{m}^{\prime}$ (and maps the root of $\mathbf{m}$ to that of $\mathbf{m}^{\prime}$ ). Note that the number and degrees of vertices, faces, edges and genus are preserved under such transformations. We will systematically identify maps that are isomorphic, making the set of maps a countable set.

We will usually denote (equivalence classes of) maps by bold lowercase letters $\mathbf{m}, \mathbf{q}, \ldots$ The standard notation for the root of a rooted map will be $e_{*}$, and it should be clear according to the context to which map $e_{*}$ refers. Also, when dealing with maps, it will often be understood that we are working with a particular graph belonging to the class of that map. This allows to consider the set of (half-)edges, vertices and faces of a (isomorphism class of a) map $\mathbf{m}$, denoted by $E(\mathbf{m}), \mathbf{E}(\mathbf{m}), V(\mathbf{m}), F(\mathbf{m})$, or simply $E, \mathbf{E}, V, F$ when there is no ambiguity.

A quadrangulation is a map whose faces all have degree 4. A map is called bipartite if its vertices can be colored in black or white, in such a way that two neighboring vertices are assigned different colors. We let $\mathbf{Q}_{g}$ be the set of rooted bipartite quadrangulation of genus $g$ (planar quadrangulations are all bipartite, but it is not the case in higher genus). For every $\mathbf{q} \in \mathbf{Q}_{g}$ we have $4|F(\mathbf{q})|=2|\mathbf{E}(\mathbf{q})|=|E(\mathbf{q})|$ and $|V(\mathbf{q})|=|F(\mathbf{q})|+\chi(g)$ by (1).

Distances in maps. For a map $\mathbf{m}$, and two vertices $x, y$, we say that the half-edges $e_{1} \ldots, e_{n}$ form a chain from $x$ to $y$ if $e_{1}^{-}=x, e_{n}^{+}=y$ and $e_{i}^{+}=e_{i+1}^{-}$for $1 \leq i \leq n-1$. We define the graph distance $d_{\mathbf{m}}(x, y)$ as the minimal $n$ such that there exist a chain of $n$ half-edges from $x$ to $y$, and a chain from $x$ to $y$ with length $d_{\mathbf{m}}(x, y)$ is called geodesic.

\subsection{Spaces of (weighted) metric spaces}

The main motivation of this paper is to study the metric structure of large random maps, endowed with their graph distance. This needs to introduce some concepts of metric geometry, essentially originating from Gromov's work [22], which have developed a growing interest in the probabilist community, starting from works of Evans and coauthors. See [15, 16, 17, 21, 26]. All results that are needed here will be presented in Section 6.

A weighted metric space is a triple $(X, d, \mu)$ where $(X, d)$ is a metric space and $\mu$ a Borel probability distribution on $(X, d)$. Two metric spaces are isometric if there exists a bijective isometry from $X$ onto $X^{\prime}$. The isometry class of the space $(X, d)$ is denoted by $[X, d]$. Two weighted metric spaces $(X, d, \mu)$ and $\left(X^{\prime}, d^{\prime}, \mu^{\prime}\right)$ are said to be isometry-equivalent if there exists a bijective isometry $\phi: X \rightarrow X^{\prime}$ such that $\phi_{*} \mu=\mu^{\prime}$. The isometry-equivalence class of $(X, d, \mu)$ is denoted by $[X, d, \mu]$.

We let $\mathbb{M}$ be the set of isometry classes of compact metric spaces, and $\mathbb{M}_{w}$ the set of isometry-equivalence classes of compact weighted metric spaces. As we will see in Section 6, it is possible to endow $\mathbb{M}_{\mathrm{w}}$ with a distance $\mathrm{d}_{\mathrm{GHP}}$, the Gromov-Hausdorff-Prokhorov distance, that makes $\left(\mathbb{M}_{\mathrm{w}}, \mathrm{d}_{\mathrm{GHP}}\right)$ a Polish space, see Theorem 6 and Proposition 8. The topology on $\mathbb{M}_{\mathrm{w}}$ will be implicitly that inherited from $\mathrm{d}_{\mathrm{GHP}}$. If $a>0$, and $\mathrm{X}=[X, d] \in \mathbb{M}$ (resp. $\mathrm{X}=[X, d, \mu] \in \mathbb{M}_{\mathrm{w}}$ ), we let $a \mathrm{X}=[X, a d]$ (resp. $a \mathrm{X}=[X, a d, \mu]$ ).

Also, a metric space $(X, d)$ is called a geodesic metric space if for which every pair of points $x, y \in X$, there exists a geodesic path between $x$ and $y$, i.e. an isometry $\gamma_{x y}:[0, d(x, y)] \rightarrow X$, 
with $\gamma_{x y}(0)=x, \gamma_{x y}(d(x, y))=y$. In particular, geodesic metric spaces are arcwise connected. For compact metric spaces, being a geodesic space is equivalent to being a path metric space, i.e. an arcwise connected metric space $(X, d)$ such that the distance $d(x, y)$ is achieved as the minimum over all continuous paths $c:[0,1] \rightarrow X$ with $c(0)=x, c(1)=y$, of the length of $c$, defined as

$$
\operatorname{length}(c)=\sup \left\{\sum_{i=0}^{n-1} d\left(c\left(t_{i}\right), c\left(t_{i+1}\right)\right): n \geq 1,0=t_{0}<t_{1}<\cdots<t_{n}=1\right\} .
$$

We let PM be the set of isometry classes of compact path (or geodesic) metric spaces.

\subsection{Main results}

Our core combinatorial result (Theorem 4) is a generalization of the Marcus-Schaeffer bijection, encoding $k$-pointed bipartite quadrangulations (with delays between sources) with the help of labeled maps with $k$ faces. The central statement in this paper is Theorem 5 (and its exceptions, Propositions 3 and 4), which allows us to take scaling limits of this simpler class of maps. We do not reproduce the exact statements of these theorems in this paragraph, as important notational background is needed.

We give several applications of these results to various (combinatorial and probabilistic) aspects of maps. The first is a counting result, giving the following asymptotic behavior for the cardinality of the set $\mathbf{Q}_{g}^{n}$ of bipartite quadrangulations of genus $g$ with $n$ faces, consistently with $[4,10]$ :

Theorem 1. - For any $g \geq 0$, it holds that

$$
\left|\mathbf{Q}_{g}^{n}\right| \underset{n \rightarrow \infty}{\sim} C_{g} 12^{n} n^{-5 \chi(g) / 4},
$$

for some constant $C_{g} \in(0, \infty)$ defined at (21).

In the case $g=0$, this result is a trivial consequence of the exact formula (see [11])

$$
\left|\mathbf{Q}_{0}^{n}\right|=\frac{2}{n+2} 3^{n} \mathrm{Cat}_{n},
$$

where $\mathrm{Cat}_{n}=\frac{1}{n+1}\left(\begin{array}{c}2 n \\ n\end{array}\right)$ is the $n$-th Catalan number, yielding the value $C_{0}=2 \pi^{-1 / 2}$.

From this statement, we see that the generating function for bipartite quadrangulations counted with respect to the number of faces has radius of convergence $12^{-1}$, and it is natural to introduce the critical Boltzmann measure $\mathbf{Q}_{g}$ on $\mathbf{Q}_{g}$ assigning mass $12^{-|F(\mathbf{q})|}$ to the element q. Since $|V(\mathbf{q})|=|F(\mathbf{q})|+\chi(g)$, the function $\beta \mapsto \mathbf{Q}_{g}\left(e^{-\beta V_{\mathbf{q}}}\right)$ is analytic in $\beta \in(0, \infty)$, where $V_{\mathbf{q}}=|V(\mathbf{q})|$ is the "volume" of $\mathbf{q}$. We define the Boltzmann-Gibbs probability distribution on $\mathbf{Q}_{g}$ with inverse temperature $\beta$ by

and

$$
\mathrm{Q}_{g}^{(\beta)}=\frac{\mathbf{Q}_{g}\left(e^{-\beta V_{\mathbf{q}}} \mathrm{d} \mathbf{q}\right)}{\mathbf{Q}_{g}\left(e^{-\beta V_{\mathbf{q}}}\right)}, \quad g \geq 1,
$$

$$
\mathrm{Q}_{0}^{(\beta)}=\frac{\mathrm{Q}_{g}\left(V_{\mathbf{q}}^{2} e^{\left.-\beta V_{\mathbf{q}} \mathrm{d} \mathbf{q}\right)}\right.}{\mathrm{Q}_{g}\left(V_{\mathbf{q}}^{2} e^{-\beta V_{\mathbf{q}}}\right)} .
$$

The reason for the difference between the cases $g=0$ and $g \geq 1$ is technical and will be explained in due course. 
With every $\mathbf{q} \in \mathbf{Q}_{g}$ we associate the metric space $\mathrm{X}_{\mathbf{q}}=\left[V(\mathbf{q}), d_{\mathbf{q}}\right]$ and the weighted space $\mathrm{X}_{\mathbf{q}}^{\mathrm{w}}=\left[V(\mathbf{q}), d_{\mathbf{q}}, \mu_{\mathbf{q}}\right]$, where

$$
\mu_{\mathbf{q}}=\frac{1}{V_{\mathbf{q}}} \sum_{v \in V(\mathbf{q})} \delta_{v}
$$

is the uniform distribution on the vertices of $\mathbf{q}$.

Theorem 2. - For every $g \geq 0$ and $\beta>0$, the probability measures

$$
\mathbf{Q}_{g}^{(\beta / a)}\left(\left\{\left(a^{-1 / 4} \mathrm{X}_{\mathbf{q}}^{\mathrm{w}}, a^{-1} V_{\mathbf{q}}\right) \in \cdot\right\}\right), \quad a>1
$$

form a relatively compact family of probability distributions on $\mathbb{M}_{\mathrm{w}} \times(0, \infty)$, endowed with the topology of weak convergence.

TheOREM 3. - A limiting point $\mathscr{S}_{g}^{(\beta)}$ of (2) as a $\rightarrow \infty$, which is a probability measure on $\mathbb{M}_{\mathrm{w}} \times(0, \infty)$, is supported on spaces $([X, d, \mu], \mathcal{V})$ such that

1. $[X, d]$ is an element of $\mathrm{PM}$,

2. $\mu$ is a diffuse measure of full support,

3. for $\mu \otimes \mu$ almost every $x, y \in X$, there exists a unique geodesic joining $x$ and $y$.

\section{Comments.}

1. Introducing the extra parameter $\beta$ and dividing it by a parameter $a \rightarrow \infty$ is referred to in physics as "approaching the critical point". One possible interpretation is to see it as a device to compute various Laplace transforms of "continuum" limits for discrete models (see also Remark 3.). It is also reminiscent of the basic method of singularity analysis consisting in approaching a singular point, and this is exactly what we do in Section 5 as a special case. On a very basic level, one should just note that letting $a \rightarrow \infty$ has the effect of making the expectation under $\mathrm{Q}_{g}^{(\beta / a)}$ of quantities such as $V_{\mathbf{q}}$ go to infinity, so that we are intuitively taking a limit as the size of the map gets large. Alternatively, we could also have considered conditioned laws of the form $\mathrm{Q}_{g}\left(\cdot \mid\left\{K^{-1} \leq a^{-1} V_{\mathbf{q}} \leq K\right\}\right)$ for some fixed $K>1$, and let $a \rightarrow \infty$.

2. Considering uniform measures on bipartite quadrangulations with $n$ faces amounts to conditioning the measure $\mathrm{Q}_{g}^{(\beta)}$ on $\{|F(\mathbf{q})|=n\}$, regardless of $\beta$, and a similar result as Theorems 2 and 3 could thus be considered as a conditioned version of these statements. We believe that such generalizations can be obtained at the price of technical complications. Note that in the case $g=0$, a result similar to Theorem 2 appears in Le Gall [26] in such a conditioned setting, though the convergence does not really take $\mu_{\mathbf{q}}$ into account (see however [26, Remark a., p. 646]).

3. As mentioned in the beginning of the article, a satisfactory statement would be to get rid of the extraction in Theorem 3, by showing that there is actually a unique limiting point to the set of measures (2). A further guess would be that this unique measure could be written in the form $\mathscr{S}_{g}\left(e^{-\beta \mathcal{V}} \mathrm{d}(\mathrm{X}, \mathcal{V})\right) / \mathscr{S}_{g}\left(e^{-\beta \mathcal{V}}\right)$ for some $\sigma$-finite measure $\mathscr{S}_{g}$ on $\mathbb{M}_{\mathrm{w}} \times(0, \infty)$.

4. Last, a natural question is to ask whether our results are universal. That is, is it still true for, say, Boltzmann-Gibbs distributions on maps of genus $g$ with faces of arbitrary valences and weight $w_{k}$ on a face of degree $k$ ? The natural generalization of $\mathrm{Q}_{g}$ would be the measure assigning weight $\prod_{f \in F(\mathbf{m})} w_{\operatorname{deg}_{\mathbf{m}} f}$ on the rooted map $\mathbf{m}$, for a particular "critical" tuning 
of the weights $w$. In this vein, the results of $[30,34,36,45]$ should be generalizable to our context.

Acknowledgments. This work was initiated while visiting the Centre for Mathematical Studies in Cambridge (UK), supported by a London Mathematical Society funding, where the question about uniqueness of geodesics arose in motivating discussions with James Norris. Thanks also to Guillaume Chapuy and Gilles Schaeffer for interesting discussions around [10]. We finally thank the two anonymous referees and the Editorial Board for very useful comments, which were greatly appreciated.

\section{Delayed Voronoi tessellations of bipartite quadrangulations}

\subsection{Fatgraphs}

As mentioned before, maps are of combinatorial nature, and there exists a purely combinatorial description of maps which will be useful to our purpose. A nice introduction to the different definitions of maps can be found in [24, Chapter 1].

A map $\mathbf{m}$ can be encoded in a triple of permutations ${ }^{(2)}$. Start from a particular representation of the map $\mathbf{m}$ on the surface $S$. Around each vertex, the outgoing half-edges are cyclically ordered in a non-ambiguous way, by considering the first intersection points of the half-edges with a small circle around the vertex, oriented counterclockwise. These cyclic orders associated with distinct vertices involve distinct elements of $E$, and thus determine a permutation $\sigma_{\mathbf{m}}$ of $E$, whose cycles are naturally associated with the vertex set $V$. We also let $\alpha_{\mathbf{m}}(e)=\bar{e}$, and $\varphi_{\mathbf{m}}=\sigma_{\mathbf{m}}^{-1} \alpha_{\mathbf{m}}^{-1}$. The triple $\left(\sigma_{\mathbf{m}}, \alpha_{\mathbf{m}}, \varphi_{\mathbf{m}}\right)$ is a fatgraph structure on $E$. We will usually drop the subscripts $\mathbf{m}$ when the situation is unambiguous.

To understand what $\varphi_{\mathbf{m}}$ is, the reader might check on examples that it corresponds to the following. Visiting the boundary of each face of the graph in counterclockwise order, determines unambiguously a cyclic ordering of the half-edges belonging to this oriented boundary. The cycles of $\varphi_{\mathbf{m}}$ are exactly the so-defined cyclic orders, which are in a one-to-one natural correspondence with faces of the graph. See Figure 1 for an illustration. One deduces that the group generated by $\left(\sigma_{\mathbf{m}}, \alpha_{\mathbf{m}}, \varphi_{\mathbf{m}}\right)$ acts transitively on $E$, amounting to the fact that maps are connected.

Conversely, every triple $(\sigma, \alpha, \varphi)$ of permutations on a finite set $E$ such that $\varphi \alpha \sigma=1, \alpha$ is an involution without fixed points, and the permutation group generated by $(\sigma, \alpha, \varphi)$ acts transitively on $E$, can be represented in the above way as the triple associated to a uniquely determined map of some genus $g$, determined by Euler's formula.

A fancy intuitive image of a fatgraph is to consider it as a road network with cars driving on the left lane. An edge and its reversal constitute the two lanes of the road, while the vertices are roundabouts connecting the roads. The cycles of $\sigma$ correspond to the outgoing roads from the roundabouts, the cycles of $\varphi$ correspond to the roads taken by a driver always taking first left at each roundabout, and the cycles of $\alpha$ correspond to a U-turn.

(2) Note that in [24], the permutations act to the right, while we prefer to make them act to the left, which results in differences like the formula $\varphi \alpha \sigma=1$ below instead of $\sigma \alpha \varphi=1$, referenced in Remark 1.3.19 in this book.

$4^{\mathrm{e}}$ SÉRIE - TOME $42-2009-\mathrm{N}^{\mathrm{o}} 5$ 


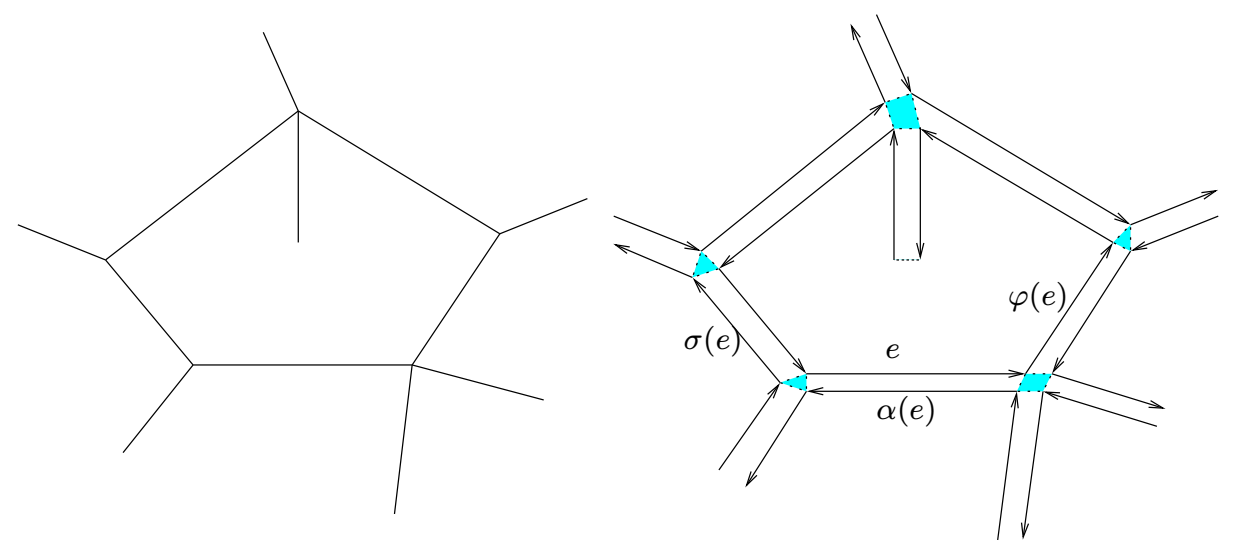

Figure 1. A portion of a map, emphasizing a face of degree 7, and an illustration of the fatgraph structure. The action of the permutations $\sigma, \alpha, \varphi$ is shown on a particular half-edge $e$.

In a fatgraph, each half-edge $e$ belongs to exactly one cycle of $\sigma$ and $\varphi$, and thus determines unambiguously a vertex and face of the map, called the vertex and face that are incident to $e$ : in terms of graphs, they correspond to the origin vertex of $e$ and the face lying to the left of $e$, respectively. By slight abuse of notation, for $f$ a face of a map, we will write $e \in f$ if the half-edge $e$ is incident to $f$, i.e. belongs to the cycle corresponding to $f$ in the fatgraph structure.

If $e_{1}, e_{2}$ are two distinct half-edges incident to the same face, we can break the cycle of $\varphi$ containing $e_{1}, e_{2}$ into two linearly ordered sequences of edges which are denoted by $\left[e_{1}, e_{2}\right]$ and $\left[e_{2}, e_{1}\right]$. Formally:

$$
\left[e_{1}, e_{2}\right]=\left\{\varphi^{i}\left(e_{1}\right): i \geq 0, e_{2} \notin\left\{\varphi^{j}\left(e_{1}\right), 0 \leq j \leq i-1\right\}\right\},
$$

so that $[e, e]=\{e\}$ with this definition. We also define $\left(e_{1}, e_{2}\right]=\left[e_{1}, e_{2}\right] \backslash\left\{e_{1}\right\}$, and so on.

Another notion that will be useful is that of corner. In a map, the corner incident to the half-edge $e$ is the intersection of a small neighborhood around the vertex incident to $e$, and the angular region comprised between $e$ and $\sigma(e)$ (to be perfectly accurate, we should define the corner as the germ of such sets). In the sequel, we will often speak indistinctly of a halfedge or its incident corner.

\subsection{Quadrangulations with sources and delays}

We first consider unrooted maps, and will re-inject the root afterwards. For $g \geq 0, k \geq 1$, we let $\mathbf{Q}_{g, k}^{\circ}$ be the set of 3-tuples of the form $(\mathbf{q}, \mathbf{x}, D)$ where

(Q1) : $\mathbf{q}$ is a bipartite (unrooted) quadrangulation of genus $g$,

(Q2): $\mathbf{x}=\left(x_{1}, \ldots, x_{k}\right)$ are elements of $V(\mathbf{q})$,

(Q3): $D=\left[d_{1}, \ldots, d_{k}\right]$ is an element of the set $\mathcal{D}(\mathbf{q}, \mathbf{x})$ of delay vectors, i.e. elements of $\mathbb{Z}^{k}$ defined modulo addition of a common integer, such that

$$
\left|d_{i}-d_{j}\right|<d_{\mathbf{q}}\left(x_{i}, x_{j}\right) \quad \text { for every } 1 \leq i \neq j \leq k,
$$


and such that

$$
d_{\mathbf{q}}\left(x_{i}, x_{j}\right)+d_{i}-d_{j} \in 2 \mathbb{N}, \quad 1 \leq i, j \leq k .
$$

When $k=1$ this definition is void, and $D=[0]$ will usually be omitted from the notation. Note that a delay vector such as in (Q3) exists if and only if $\min _{i \neq j} d_{\mathbf{q}}\left(x_{i}, x_{j}\right) \geq 2$. Indeed, in that case we can take a bicoloration of $V(\mathbf{q})$ in black and white, and notice that two vertices are at even distance if and only if they are of the same color. Setting $d_{i}=0$ if $x_{i}$ is white, and $d_{i}=1$ if $x_{i}$ is black, yields a vector $\left(d_{1}, \ldots, d_{k}\right)$ with the wanted property. Conversely, if $d\left(x_{i}, x_{j}\right)=1$ for some $i \neq j$, then obviously we should have $d_{i} \neq d_{j}$ and $\left|d_{i}-d_{j}\right|<1$ which is impossible. This implies some restrictions on the quadrangulations involved in $\mathbf{Q}_{g, k}^{\circ}$ - for instance, they should have at least $k$ distinct vertices.

With any element of $\mathbf{Q}_{g, k}^{\circ}$, we can associate a partition of the set of edges of $\mathbf{q}$ that is very much reminiscent of Voronoi tessellations in a Euclidean space. We fix arbitrarily a representative of a delay vector $\left(d_{1}, \ldots, d_{k}\right)$, and define $\mathbf{l}_{i}(x)=d_{\mathbf{q}}\left(x, x_{i}\right)+d_{i}$ for $x \in V(\mathbf{q})$. Then for $1 \leq i \leq k$, and for $x$ and $y$ adjacent in $\mathbf{q}$,

$$
\left|\mathbf{l}_{i}(x)-\mathbf{l}_{i}(y)\right|=1,
$$

since this quantity can be at most one and vertices are at even distance of $x_{i}$ if and only if they have the same color in a bipartite coloration of the vertices. Thus, defining

$$
\mathbf{l}(x)=\min _{i}\left(d_{i}+d_{\mathbf{q}}\left(x, x_{i}\right)\right)
$$

we deduce that $|\mathbf{l}(x)-\mathbf{l}(y)| \leq 1$ for every adjacent $x, y$.

On the other hand, by (Q3) we have $d\left(x_{1}, x_{i}\right)+d_{1}-d_{i} \in 2 \mathbb{Z}$, which shows that $\mathbf{l}_{i}\left(x_{1}\right)-\mathbf{l}_{j}\left(x_{1}\right) \in 2 \mathbb{Z}$ for every $i, j$. By (5) and since the graph is connected, this property propagates from $x_{1}$ to all other vertices, so it holds that $\mathbf{l}_{i}-\mathbf{l}_{j}$ takes its values in $2 \mathbb{Z}$ for every $i, j$. Consequently, we cannot have $\mathbf{l}_{i}(x)=\mathbf{l}_{j}(y)$ with adjacent $x, y$ for any $i, j$, and this implies that $\mathbf{l}(x) \neq \mathbf{l}(y)$ for adjacent $x, y$. Therefore

$$
|\mathbf{l}(x)-\mathbf{l}(y)|=1, \quad \text { for all adjacent } x, y .
$$

This allows us to fix an orientation $E_{1 / 2} \subset E(\mathbf{q})$ by enforcing $\mathbf{l}\left(e^{+}\right)=\mathbf{l}\left(e^{-}\right)-1$ for all $e \in E_{1 / 2}$. The function 1 decreases along chains of edges of $E_{1 / 2}$, and plainly, the only vertices without edges pointing outwards are $x_{1}, \ldots, x_{k}$. Thus, any chain of edges of $E_{1 / 2}$ can be extended into a maximal chain ending at some element of $\left\{x_{1}, \ldots, x_{k}\right\}$. Also, any such chain with initial vertex $x$ is geodesic, and the endpoint of a maximal extension must be a $x_{i}$ with $i$ such that $\mathbf{l}(x)=\mathbf{l}_{i}(x)$. To see this, recall that each of the functions $\mathbf{l}_{i}$ makes a step in $\{-1,1\}$ when passing from a vertex to one of its neighbors, by (5). Moreover, we just saw that the function 1 decreases by 1 when going along one step in the chain. So if the chain terminates at $x_{i}$, it must hold that $\mathbf{l}(y)=\mathbf{l}_{i}(y)$ for every vertex $y$ along the chain. Since $\mathbf{l}(x)=d_{\mathbf{q}}\left(x_{i}, x\right)+d_{i}$ and $\mathbf{l}\left(x_{i}\right)=d_{i}$, the chain has length $d_{\mathbf{q}}\left(x_{i}, x\right)$ and is a geodesic.

We color each edge of $E_{1 / 2}$ with one in $k$ colors in the following way. For $e \in E_{1 / 2}$, consider the maximal path starting with $e$ and turning as much to the left as possible, that is, after arriving at a vertex, it takes the first outgoing edge it encounters in clockwise order around the vertex. If this path ends at vertex $x_{i}$, assign the color $i$ to the edge $e$. 
Definition 1. - The set $E_{i}(\mathbf{q}, \mathbf{x}, D)$ of edges colored $i$ is called the $D$-delayed Voronoi tile with sources $\mathbf{x}$ and center $x_{i}$.

The $D$-delayed Voronoi tiles form a partition of $E_{1 / 2}$. The reason for the name is that when $D=0$, the set $E_{i}(\mathbf{q}, \mathbf{x}, 0)$ contains all the edges pointing from a vertex that is strictly closer to $x_{i}$ than any other source, which is the usual property of Voronoi tiles in Euclidean spaces. A general delay vector $D$ corresponds to the fact that distances from different sources are measured with a relative advantage of $d_{i}-d_{j}$ of source $x_{j}$ on source $x_{i}$. Note that it might be that no edge pointing from a vertex $x$ for which $d_{\mathbf{q}}\left(x, x_{i}\right)=\min _{j} d_{\mathbf{q}}\left(x, x_{j}\right)$ lies in $E_{i}(\mathbf{q}, \mathbf{x}, 0)$. In fact, it holds that if

$$
V_{i}(\mathbf{q}, \mathbf{x}, D)=\left\{x_{i}\right\} \cup\left\{e^{-}: e \in E_{i}(\mathbf{q}, \mathbf{x}, D)\right\}, \quad 1 \leq i \leq k,
$$

then these sets cover $V(\mathbf{q})$ and we have

$$
\left\{x \in V(\mathbf{q}): \mathbf{l}_{i}(x)=\mathbf{l}(x)<\min _{j \neq i} \mathbf{l}_{j}(x)\right\} \subsetneq V_{i}(\mathbf{q}, \mathbf{x}, D) \subseteq\left\{x \in V(\mathbf{q}): \mathbf{l}_{i}(x)=\mathbf{l}(x)\right\} .
$$

It is not difficult to see that the first inclusion is always strict as soon as $k \geq 2$, because one can find some $x \in V_{i}(\mathbf{q}, \mathbf{x}, D)$ and some $j \neq i$ such that $x \in V_{j}(\mathbf{q}, \mathbf{x}, D)$, and therefore it holds that $\mathbf{l}_{i}(x)=\mathbf{l}_{j}(x)=\mathbf{l}(x)$. The second inclusion can be strict, although this is harder to see at first: on Figure 2, the only vertex $x$ with label 3 is at distance 3 of $x_{1}$ and 2 of $x_{2}$, and $d_{1}=0, d_{2}=1$, so that $\mathbf{l}_{1}(x)=\mathbf{l}_{2}(x)=\mathbf{l}(x)$, however, $x$ does not belong to $V_{1}(\mathbf{q}, \mathbf{x}, D)$, because the (only) leftmost geodesic started from $x$ ends at $x_{2}$.

\subsection{The bijection}

An embedded graph is said to be labeled if its vertices are assigned integer values $\mathbf{l}=(\mathbf{l}(x), x \in V)$, in such a way that $|\mathbf{l}(x)-\mathbf{l}(y)| \leq 1$ for every adjacent $x, y$. Labeled maps are defined accordingly. We let $\mathbf{L M}_{g, k}^{\circ}$ be the set of pairs of the form $(\mathbf{m},[\mathbf{l}])$ such that

(LM1): $\mathbf{m}$ is a map of genus $g$ with $k$ faces indexed as $f_{1}(\mathbf{m}), \ldots, f_{k}(\mathbf{m})$

(LM2) : $\mathbf{l}=(\mathbf{l}(x), x \in V(\mathbf{m}))$ is a labeling of $\mathbf{m}$, and [1] is its class up to an additive constant in $\mathbb{Z}$.

Our goal in the remaining of this section is to construct and study a bijective mapping $\Psi_{g, k}^{\circ}$ between $\mathbf{Q}_{g, k}^{\circ}$ and $\mathbf{L} \mathbf{M}_{g, k}^{\circ}$, and its inverse mapping $\Phi_{g, k}^{\circ}$. We first make some preliminary remarks.

1. The construction starts from a particular embedding of an element of $\mathbf{Q}_{g, k}^{\circ}$, and builds an embedding of the image labeled map, by deletion of some of the edges and vertices of the initial quadrangulation, and addition of new edges.

In particular, if $(\mathbf{q}, \mathbf{x}, D)$ and $(\mathbf{m},[\mathbf{l}])$ correspond by the construction, the set of vertices of $\mathbf{m}$ will be identified with the set $V(\mathbf{q}) \backslash\left\{x_{1}, \ldots, x_{k}\right\}$ of vertices of $\mathbf{q}$ distinct from the sources. This will be important in the sequel. This is also the reason why we will give the same name 1 to labeling functions on a quadrangulation $\mathbf{q}$ and its image map $\mathbf{m}$, as the labeling function on $\mathbf{m}$ is indeed the restriction to the vertices of $\mathbf{m}$ of the labeling function $\mathbf{l}$ on $\mathbf{q}$ introduced above. 

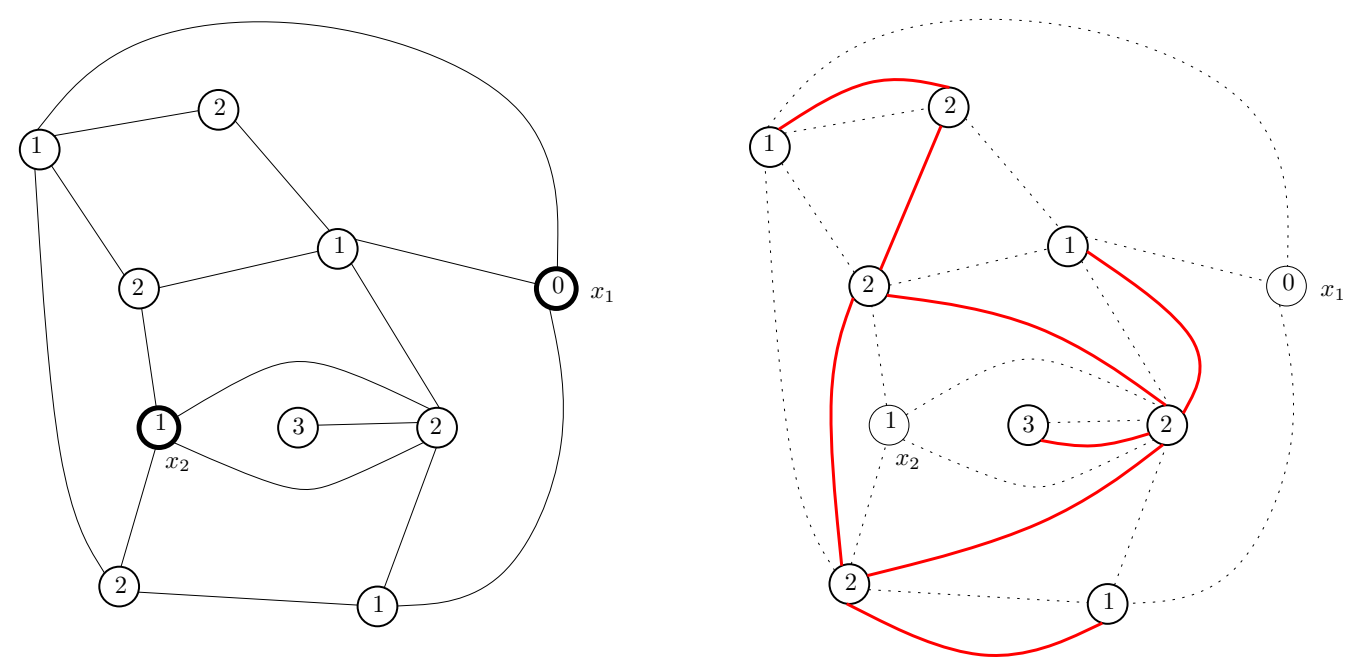

Figure 2. An example of a 2-pointed quadrangulation of genus 0 , with delays $d_{1}=0, d_{2}=1$ (left) and the associated labeled map with 2 faces by $\Psi_{0,2}^{\circ}$

2. The mapping $\Psi_{g, k}^{\circ}$ is a natural analog of a bijection of Schaeffer between pointed planar maps and well-labeled trees [7,11]. More precisely, in the case $(g, k)=(0,1)$, the set $\mathbf{L} \mathbf{M}_{0,1}^{\circ}$ is constituted of labeled (unrooted) planar trees, which themselves are in one-to-one correspondence with well-labeled trees, for which all labels are positive and at least one vertex has label 1 , by taking any labeling $\mathbf{l}$ and adding $-\min \mathbf{l}+1$. A generalization of Schaeffer's bijection to genus $g \geq 1$ is discussed in Marcus \& Schaeffer [33]. It is recovered as the special case $k=1$ of our construction, where the set $\mathbf{L M}_{g, 1}^{\circ}$ is constituted of labeled maps of genus $g$ with only one face, called $g$-trees in [33].

3. We can understand our generalized bijection as running simultaneously the MarcusSchaeffer construction at $k$ distinct competing vertices. Informally, let water flow at unit speed from the sources $x_{1}, \ldots, x_{k}$, in such a way that the water starts diffusing from $x_{i}$ at time $d_{i}$, and takes unit time to go through an edge. When water currents emanating from different edges meet at a vertex (whenever the water initially comes from the same source or from different sources), they can go on flowing into unvisited edges only by respecting the rules of a roundabout, i.e. edges that can be attained by turning around the vertex counterclockwise and not crossing any other current. The process ends when the water cannot flow anymore, and the tile $E_{i}(\mathbf{q}, \mathbf{x}, D)$ is the set of reversed half-edges that are visited by the water flowing from source $x_{i}$. The parity condition on $D$ implies that the water flows emanating from different sources never meet in the middle of an edge, but always at vertices.

We now give the rigorous construction, and refer to Figure 2 for an example.

\subsection{Construction of the bijection}

We start from an element $(\mathbf{q}, \mathbf{x}, D) \in \mathbf{Q}_{g, k}^{\circ}$, and take some particular embedding of $\mathbf{q}$. Choosing arbitrarily $\left(d_{1}, \ldots, d_{k}\right) \in D$, we label the vertices by the function $\mathbf{l}: x \in V(\mathbf{q}) \mapsto \min _{i}\left(d_{i}+d_{\mathbf{q}}\left(x, x_{i}\right)\right)$. By (5), the labels of vertices encountered when 

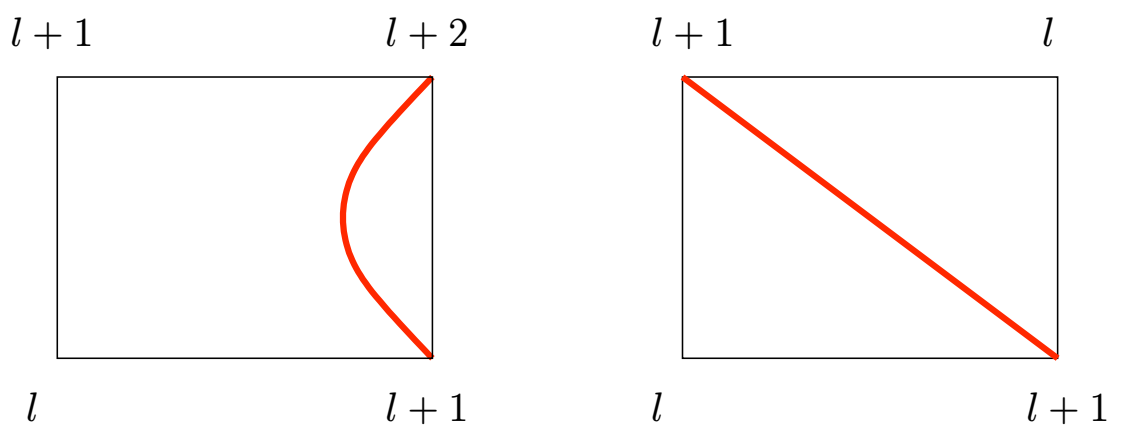

Figure 3. Adding new edges (thick lines) inside simple and confluent faces of a graphical representation of an element of $\mathbf{Q}_{g, k}^{\circ}$

exploring a face of $\mathbf{q}$, starting from the vertex with lowest label and turning counterclockwise, must be either of the form $l, l+1, l+2, l+1$ or $l, l+1, l, l+1$. Following [33], we call faces simple and confluent accordingly. We then perform the construction of [33] on this labeled object, that is, we add extra edges to the map $\mathbf{m}$, one inside each face:

- if the face is simple, then the added edge links the vertex with highest label to its successor in clockwise order around the face, hence splitting the face into a face with degree 4 , and a face of degree 2

- if the face is confluent, then the added edge links the two vertices with highest label, hence splitting the face into two triangular faces.

In this way, we have defined a new map (graph) $\mathbf{q}^{\prime}$ whose faces have degree 2,3 or 4 , and with labeled vertices, whose set is still $V(\mathbf{q})$. See Figure 3.

The newly added edges, excluding the ones of the original map, define a graph $\mathbf{m}$ on the surface on which $\mathbf{q}$ is drawn. It is easily obtained from the construction that the vertex set of this new graph is exactly $V(\mathbf{q}) \backslash\left\{x_{1}, \ldots, x_{k}\right\}$. Indeed, the vertices $x_{1}, \ldots, x_{k}$ have neighbors with strictly larger labels, and thus have the least labels in any face they are incident to, and cannot be incident to a newly added edge. Conversely, if $x$ is a vertex not in $\left\{x_{1}, \ldots, x_{k}\right\}$, with label $\mathbf{l}(x)=l$, we let $e_{1}, \ldots, e_{r}$ be the outgoing edge cycle associated with $x$ in the fatgraph structure of $\mathbf{q}$. Then there exists $i$ such that $\mathbf{l}\left(e_{i}^{+}\right)=l-1$. It is then easy that the new edge added in the face of $\mathbf{q}$ incident to $\bar{e}_{i}$ (i.e. located to the right of $e_{i}$ ) must be incident to $x$, regardless of the face being simple or confluent.

Lemma 1. - The embedded graph $\mathbf{m}$ is a map. Each face of $\mathbf{m}$ contains exactly one element of $\left\{x_{1}, \ldots, x_{k}\right\}$, and is indexed accordingly as $f_{1}(\mathbf{m}), \ldots, f_{k}(\mathbf{m})$.

Once this is proved, and recalling that vertices of $\mathbf{q}$ are vertices of $\mathbf{m}$ as well, we use $\mathbf{l}=\left(\mathbf{l}(x), x \in V(\mathbf{q}) \backslash\left\{x_{1}, \ldots, x_{k}\right\}\right)$, as a labeling function on $V(\mathbf{m})$. It is now straightforward to check that $\Psi_{g, k}^{\circ}(\mathbf{q}, \mathbf{x}, D):=(\mathbf{m},[\mathbf{l}])$ satisfies properties (LM1,LM2).

Proof of Lemma 1. - We proceed in a similar way as in [33]. Namely, we consider the dual edges of $\mathbf{q}^{\prime}$ that do not cross the newly added edges, and give them an orientation according to Figure 4, i.e. in such a way that the vertex located to the right of the oriented dual edge 

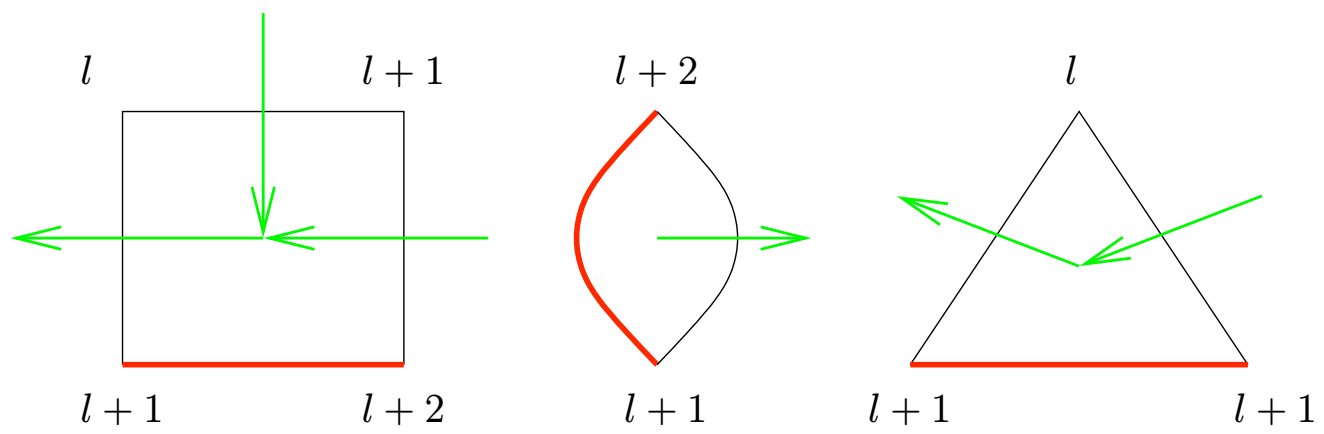

Figure 4. Drawing and orienting the dual edges not crossing the newly added edges inside each of the possible types of faces of $\mathbf{q}^{\prime}$

has strictly smaller label than the one located to the left. In doing so, we create an oriented spanning subgraph of the dual graph of $\mathbf{q}^{\prime}$, call $E_{1 / 2}^{\circ}$ the set of its edges.

By Figure 4, for every face $f$ of $\mathbf{q}^{\prime}$, there is a single element of $E_{1 / 2}^{\circ}$ that goes out of it. Therefore, there is a unique, infinite chain of $E_{1 / 2}^{\circ}$, say $\left(e_{m}^{\circ}, m \geq 1\right)$, starting from $f$. This infinite chain eventually cycles. By inspection of Figure 4 , if $l_{i}$ is the label of the vertex of q located to the right of $e_{m}^{\circ}$, then it must hold that $l_{m} \geq l_{m+1}$ for every $m \geq 1$. But since $\left(e_{m}^{\circ}, m \geq 1\right)$ eventually cycles, the sequence $\left(l_{m}, m \geq 1\right)$ is eventually constant, equal to $l$ say. Another look at the first and third situations of Figure 4 shows that the only possibility is that the cycle (i.e. the sequence $e_{m}^{\circ}, m \geq N$ for $n$ large enough) surrounds a single vertex of q. This vertex must be an element of the set of sources $\left\{x_{1}, \ldots, x_{k}\right\}$, since these are the only vertices of $\mathbf{q}^{\prime}$ that are incident only to edges of $\mathbf{q}$, and hence that can be surrounded by a cycle of $E_{1 / 2}^{\circ}$.

This implies that the (oriented) graph $E_{1 / 2}^{\circ}$ has $k$ distinct connected components, and hence $\mathbf{m}$ has $k$ faces. Now starting from the union of faces and edges of $\mathbf{q}^{\prime}$ that are incident to $x_{i}$, which is a topological disk because $x_{i}$ is incident exactly once to each face, we can add the faces of $\mathbf{q}^{\prime}$ leading to $x_{i}$ in the dual path of edges of $E_{1 / 2}^{\circ}$ without ever making a cycle, so that the faces of $\mathbf{m}$ are simply connected, hence $\mathbf{m}$ is a map.

\subsection{Converse construction}

In the sequel, the label $\mathbf{l}(e)$ of a half-edge (or the associated corner) $e$ will be a notational shorthand for the label of its origin $\mathbf{l}\left(e^{-}\right)$.

The idea of the converse construction is simply to perform the Marcus-Schaeffer inverse construction separately inside each face of $\mathbf{m}$. Specifically, given $(\mathbf{m},[\mathbf{l}])$, we add an extra vertex $x_{i}$ inside the $i$-th face of $\mathbf{m}$. We choose an arbitrary labeling $\mathbf{l} \in[\mathbf{l}]$ and extend it to $V(\mathbf{m}) \cup\left\{x_{1}, \ldots, x_{k}\right\}$ by

$$
\mathbf{l}\left(x_{i}\right)=\min _{e \in f_{i}(\mathbf{m}): e^{-}=x} \mathbf{l}(e)-1 .
$$

We let $(\sigma, \alpha, \varphi)$ be the permutation 3-tuple associated with the fatgraph structure on $\mathbf{m}$.

We define the successor $s(e)$ of an edge $e \in f_{i}(\mathbf{m})$ by $s(e)=x_{i}$ if $\mathbf{l}(e)=\mathbf{l}\left(x_{i}\right)+1$, and otherwise,

$$
s(e)=\varphi^{m(e)}(e),
$$


where

$$
m(e)=\min \left\{m \geq 1: \mathbf{l}\left(\varphi^{m}(e)\right)=\mathbf{l}(e)-1\right\} .
$$

We then draw an edge between the corners incident to each edges $e$ and $s(e)$ for each $e \in E(\mathbf{m})$, in such a way that these edges do not cross each other. This is possible, because by the constraint on the labels, we have $\left|\mathbf{l}\left(\varphi^{m}(e)\right)-\mathbf{l}\left(\varphi^{m+1}(e)\right)\right| \leq 1$, which entails that if $e$ has successor $s(e) \notin\left\{x_{1}, \ldots, x_{k}\right\}$ and $e^{\prime} \in[e, s(e)]$, then we have $\mathbf{l}\left(e^{\prime}\right) \geq \mathbf{l}(e)$ and thus $s\left(e^{\prime}\right)$ must be in $\left(e^{\prime}, s(e)\right]$. If $s(e)=x_{i}$ then $e$ has minimal label inside the face it is incident to, and the labeling constraint prevents $e$ from being in any set of the form $\left[e^{\prime}, s\left(e^{\prime}\right)\right]$.

When the new edges have been added in this proper non-crossing way, we delete the edges of $\mathbf{m}$ and keep only the newly added ones.

\section{Lemma 2. - The following properties hold.}

1. The graph thus obtained is a map $\mathbf{q}$, which is a bipartite quadrangulation.

2. The labels $(\mathbf{l}(x), x \in V(\mathbf{q}))$ are equal to $\mathbf{l}(x)=\min _{1 \leq i \leq k}\left(d_{\mathbf{q}}\left(x_{i}, x\right)+d_{i}\right)$, where $d_{i}=\mathbf{l}\left(x_{i}\right)$.

3. Setting $D=\left[d_{1}, \ldots, d_{k}\right]$, the triple $(\mathbf{q}, \mathbf{x}, D)$ is an element of $\mathbf{Q}_{g, k}^{\circ}$.

4. The set of oriented edges of $\mathbf{q}$ from e to $s(e)$, for $e \in f_{i}(\mathbf{m})$, is $E_{i}(\mathbf{q}, \mathbf{x}, D)$.

Proof. - We first check property 1 . Let $e$ be an edge of $\mathbf{m}$ with label $\mathbf{l}(e)=l$. Its successor $s(e)$ has label $l-1$. If $\varphi(e)$ has also label $l$, then its successor $s(\varphi(e))=s(e)$, so that the edges between $e, \varphi(e)$ and their common successor form a triangle with the base edge $e$. This is the same in the face incident to the reversal $\bar{e}$, so that the deletion of $\{e, \bar{e}\}$ after adding the new edges yields a 4 -valent face.

If $\varphi(e) \neq l$, then either $\varphi(e)$ has label $l+1$, or $\varphi(e)=s(e)$ by definition of the successor. In the first case, notice that we also have that $s(s(\varphi(e)))=s(e)$, because $s(\varphi(e))$ must have label $l$ and lie in $[e, s(e)]$. Thus, the newly added edges between (the corners associated with) $e$ and $s(e), \varphi(e)$ and $s(\varphi(e))$, and $s(\varphi(e))$ and $s(e)$, must form a 4-valent face with the edge $e$. Now, in the face $\bar{e}$ is incident to, observe that the successor of $\bar{e}$ must be $\sigma^{-1}(e)=\varphi(\bar{e})$, as they are successive vertices with labels $l+1$ and $l$. Thus, the newly added edges around the edges $e, \bar{e}$ form a 4 -valent face. It remains to treat the case where $s(e)=\varphi(e)$, but then we are in the situation just discussed in the face incident to $\bar{e}$.

Therefore, any (non-oriented) edge of $\mathbf{m}$ is surrounded by a 4 -valent face in the new graph, and these are the only possible faces, as any new edge is drawn from some corner of $\mathbf{m}$. Thus, the new graph is a map on the same surface as $\mathbf{m}$. It has only square faces, and coloring vertices according to the parity of their labels yields a bipartite coloration.

To check property 2 , let $x$ be a vertex of $\mathbf{m}$ incident to the face $f_{i}(\mathbf{m})$, with label $l$, and let $e \in f_{i}(\mathbf{m})$ be an edge pointing out from $x$. Then the (half)-edges drawn between the corners $e, s(e), s^{2}(e), \ldots, x_{i}$ form a chain with length $\mathbf{l}(x)-\mathbf{l}\left(x_{i}\right)$, and by definition, it turns to the left as much as possible among the half-edges of $\mathbf{q}$ along which $\mathbf{l}$ decreases. Moreover, any path using only the edges in q must be at least as long, because these edges always link vertices with labels that differ by 1 exactly. Hence, this path is a geodesic and one deduces $d_{\mathbf{q}}\left(x_{i}, v\right)+d_{i}=l$. For the same reason, we must have $d_{\mathbf{q}}\left(x_{j}, v\right)+d_{j} \geq l$ for every $j \in\{1, \ldots, k\}$, so that $l=\min _{j}\left(d_{\mathbf{q}}\left(v, x_{j}\right)+d_{j}\right)$. This gives, in passing, that the edge of $\mathbf{q}$ from $e$ to $s(e)$ is an element of $E_{i}(\mathbf{q}, \mathbf{x}, D)$, yielding the property 4 . 
We now check that $D=\left[d_{1}, \ldots, d_{k}\right]$ is a delay vector, which will entail property 3 . First, we have seen that for any vertex $x$, the length of a shortest path from $x_{i}$ to $x_{j}$ passing through $x$ must have length at least $2 \mathbf{l}(x)-d_{i}-d_{j}$. Hence $d_{\mathbf{q}}\left(x_{i}, x_{j}\right)$ is at least $2 l_{*}-d_{i}-d_{j}$, where $l_{*}$ is the maximal label on a geodesic path from $x_{i}$ to $x_{j}$. Thus $d_{\mathbf{q}}\left(x_{i}, x_{j}\right)$ is strictly larger than $\left|d_{i}-d_{j}\right|$ if $l_{*}>d_{i} \vee d_{j}$. Obviously this is the case since the $x_{i}$ 's are pairwise distinct and have neighboring vertices with strictly larger labels, by construction.

Finally, we infer that $d_{\mathbf{q}}\left(x_{i}, x_{j}\right)+d_{i}-d_{j} \in 2 \mathbb{N}$ from the fact that $x_{i}$ can be colored according to the parity of $d_{i}-d_{j}$. If $x_{i}$ and $x_{j}$ have same color, then they are at even distance and $d_{i}-d_{j}$ is even, and otherwise both $d_{\mathbf{q}}\left(x_{i}, x_{j}\right)$ and $d_{i}-d_{j}$ are odd.

Note that, due to property 4 of Lemma 2, the set of half-edges of $\mathbf{m}$ is identified with the set $E_{1 / 2}$ of edges of $\mathbf{q}$ with the special orientation of Section 2.2, in such a way that halfedges of $\mathbf{m}$ incident to $f_{i}(\mathbf{m})$ correspond to elements of $E_{i}(\mathbf{q}, \mathbf{x}, D)$. This will be useful in the sequel.

We now state the main result of this section.

Theorem 4. - Let $k \geq 1$ and $g \geq 0$. The mappings $\Psi_{g, k}^{\circ}$ and $\Phi_{g, k}^{\circ}$ are inverse of each other, and therefore are bijections between the sets $\mathbf{Q}_{g, k}^{\circ}$ and $\mathbf{L M}_{g, k}^{\circ}$. Moreover, if $(\mathbf{m},[\mathbf{l}])=\Psi_{g, k}^{\circ}(\mathbf{q}, \mathbf{x}, D)$,

1. $|F(\mathbf{q})|=|\mathbf{E}(\mathbf{q})| / 2=|\mathbf{E}(\mathbf{m})|$, more precisely $\left|E_{i}(\mathbf{q}, \mathbf{x}, D)\right|=\operatorname{deg}_{\mathbf{m}}\left(f_{i}(\mathbf{m})\right), 1 \leq i \leq k$.

2. $|V(\mathbf{q})|=|V(\mathbf{m})|+k$,

3. For every $1 \leq i \leq k$ and $d \geq 1$,

$$
\left|\left\{e \in E_{i}(\mathbf{q}, \mathbf{x}, D): d_{\mathbf{q}}\left(e^{-}, x_{i}\right)=d\right\}\right|=\left|\left\{e \in f_{i}(\mathbf{m}): \mathbf{l}(e)-\min _{e^{\prime} \in f_{i}(\mathbf{m})} \mathbf{l}\left(e^{\prime}\right)+1=d\right\}\right|
$$

(note that the last appearing quantity is independent of the choice of $\mathbf{l} \in[\mathbf{l}]$ ).

Properties 1,2,3 are easy consequences of the construction and Lemma 2. They are far from being the only important properties of $\Psi_{g, k}^{\circ}$. Other consequences will be derived in Section 2.7.

Proof. - It is easy to see why $\Psi_{g, k}^{\circ}\left(\Phi_{g, k}^{\circ}(\mathbf{m},[\mathbf{l}])\right)=(\mathbf{m},[\mathbf{l}])$ from the construction of the 4 -valent faces of $\Phi_{g, k}^{\circ}(\mathbf{m},[\mathbf{l}])$ depicted in the previous proof. We just notice that in each of the three cases that are detailed $\left(e \in E(\mathbf{m})\right.$ is such that $\mathbf{l}\left(e^{-}\right)=\mathbf{l}\left(e^{+}\right), \mathbf{l}\left(e^{-}\right)=\mathbf{l}\left(e^{+}\right)+1$ or $\left.\mathbf{l}\left(e^{-}\right)=\mathbf{l}\left(e^{+}\right)-1\right)$, the edge $e$ that is deleted in the end is precisely re-added by the construction of $\Psi_{g, k}^{\circ}$ (the first case is that of a confluent face, and the two others correspond to simple faces). The labels remain unchanged in this step thanks to the last sentence of Lemma 2.

We now prove that $\Phi_{g, k}^{\circ}\left(\Psi_{g, k}^{\circ}(\mathbf{q}, \mathbf{x}, D)\right)=(\mathbf{q}, \mathbf{x}, D)$. To see this we must check that the edges of $\mathbf{q}$ can all be obtained as edges between a corner and its successor in $\Psi_{g, k}^{\circ}(\mathbf{q}, \mathbf{x}, D)=(\mathbf{m},[\mathbf{l}])$. Necessarily, edges must join corners with labels differing by 1 , and cannot cross the edges of $\mathbf{m}$, so that they all lie inside some of its faces. Moreover, inside the face $f_{i}(\mathbf{m})$, all corners with label $d_{i}+1$ must be re-linked to the vertex $x_{i}$. So let $e, e^{\prime} \in f_{i}(\mathbf{m})$ be linked by an edge of $\mathbf{q}$, for which $\mathbf{l}(e)=\mathbf{l}\left(e^{\prime}\right)+1=l+1$, but such that $s(e) \neq e^{\prime}$. We choose moreover $e, e^{\prime}$ in such a way that $l$ is minimal, that is, all the edges between a corner with label $\leq l$ and its successor are edges of $\mathbf{q}$. In this case, the paths $s(e), s^{2}(e), \ldots, x_{i}$ and $e^{\prime}, s\left(e^{\prime}\right), \ldots, x_{i}$ are paths in $\mathbf{q}$, and cannot cross the edge between $e$ and $e^{\prime}$. On the other hand, $s(e), s^{2}(e), \ldots$ 
must lie in $\left[e, e^{\prime}\right]$, while $s\left(e^{\prime}\right), s^{2}\left(e^{\prime}\right), \ldots$ must all lie in $\left[e^{\prime}, e\right]$. In particular, adding the edge between $e$ and $e^{\prime}$ to $f_{i}(\mathbf{m})$ breaks the latter in two connected regions, both of which have to contain $x_{i}$, and this is impossible.

\subsection{Rooting}

We let $\mathbf{Q}_{g, k}$ be the set of 3-tuples $(\mathbf{q}, \mathbf{x}, D)$, where $\mathbf{q}$ is an element of $\mathbf{Q}_{g}$, and if $\mathbf{q}^{\circ}$ denotes the associated unrooted map, $\left(\mathbf{q}^{\circ}, \mathbf{x}, D\right) \in \mathbf{Q}_{g, k}^{\circ}$. Similarly, we let $\mathbf{L M}_{g, k}$ be the set of pairs $(\mathbf{m},[\mathbf{l}])$ where $\left(\mathbf{m}^{\circ},[\mathbf{l}]\right) \in \mathbf{L M}_{g, k}^{\circ}$.

Let $(\mathbf{q}, \mathbf{x}, D) \in \mathbf{Q}_{g, k}$, and $\left(\mathbf{m}^{\circ},[\mathbf{l}]\right)=\Psi_{g, k}^{\circ}\left(\mathbf{q}^{\circ}, \mathbf{x}, D\right)$. With root edge $e_{*}$ of $\mathbf{q}$, corresponds naturally one of the corners of $(\mathbf{m},[\mathbf{l}])$, i.e. it is the unique corner such that the edge drawn between $e$ and $s(e)$ is either equal to $e_{*}$ or its reversal $\bar{e}_{*}$ when performing the converse construction. We root $\mathbf{m}^{\circ}$ at this corner, obtaining a rooted map $\mathbf{m}$, and let $\Psi_{g, k}(\mathbf{q}, \mathbf{x}, D)=(\mathbf{m},[\mathbf{l}])$. Notice that since any edge of $\mathbf{q}$ joins two vertices with labels that differ by 1 , the two possible orientations of a base edge of $\mathbf{q}$ yield two distinct rooted and $k$-pointed quadrangulations $(\mathbf{q}, \mathbf{x})$. The following corollary of Theorem 4 follows.

Corollary 1. - The mapping $\Psi_{g, k}: \mathbf{Q}_{g, k} \rightarrow \mathbf{L M}_{g, k}$ is two-to-one.

Besides getting rid of symmetries, the nice feature of rooting is that it will allow us to choose a particular labeling function inside its class, as we will see in Section 3.

\subsection{Bounds on distances}

The construction of $\Phi_{g, k}^{\circ}$ allows us to get useful bounds on distances between vertices in the quadrangulation, from the label function on the associated map.

Lemma 3. - Let $(\mathbf{q}, \mathbf{x}, D) \in \mathbf{Q}_{g, k}$ and $(\mathbf{m},[\mathbf{l}])=\Psi_{g, k}(\mathbf{q}, \mathbf{x}, D)$. Take two half-edges $e_{1}, e_{2} \in E_{i}(\mathbf{q}, \mathbf{x}, D)$ for some $i$. Still denoting by $e_{1}, e_{2}$ the half-edges of $f_{i}(\mathbf{m})$ associated with $e_{1}, e_{2}$ by $\Psi_{g, k}$, we have

$$
\left|\mathbf{l}\left(e_{1}\right)-\mathbf{l}\left(e_{2}\right)\right| \leq d_{\mathbf{q}}\left(e_{1}^{-}, e_{2}^{-}\right) \leq \mathbf{l}\left(e_{1}\right)+\mathbf{l}\left(e_{2}\right)-2 \min _{e \in\left[e_{1}, e_{2}\right]} \mathbf{l}(e)+2
$$

(here the interval $\left[e_{1}, e_{2}\right]$ is the one in $\mathbf{m}$ ).

Note that we could have interchanged the roles of $e_{1}$ and $e_{2}$, so that we have in fact

$$
d_{\mathbf{q}}\left(e_{1}^{-}, e_{2}^{-}\right) \leq \mathbf{l}\left(e_{1}\right)+\mathbf{l}\left(e_{2}\right)-2 \max \left(\min _{e \in\left[e_{1}, e_{2}\right]} \mathbf{l}(e), \min _{e \in\left[e_{2}, e_{1}\right]} \mathbf{l}(e)\right)+2 .
$$

Proof. - The lower bound is obvious from the fact that for $e \in E_{i}(\mathbf{q}, \mathbf{x}, D)$, $\mathbf{l}(e)-\mathbf{l}\left(x_{i}\right)=d_{\mathbf{q}}\left(e^{-}, x_{i}\right)$ and by the triangular inequality. For the upper bound, consider the last half-edge $e$, among the sequence $s^{m}\left(e_{1}\right), m \geq 0$, that lies in $\left[e_{1}, e_{2}\right]$. Necessarily, $\mathbf{l}(e) \leq \mathbf{l}\left(e_{2}\right)$ as otherwise, the successor of $e$ would be in $\left[e, e_{2}\right]$. From this, it follows that $s(e)=s^{\mathbf{l}\left(e_{2}\right)-\mathbf{l}(e)+1}\left(e_{2}\right)$. Therefore, there exists a chain in $\mathbf{q}$ from $e_{1}^{-}$to $e_{2}^{-}$with length $\left(\mathbf{l}\left(e_{1}\right)-\mathbf{l}(e)\right)+1+\left(\mathbf{l}\left(e_{2}\right)-\mathbf{l}(e)+1\right)$, which yields the result. 


\section{Decomposition of labeled maps}

Our next task is to make one more step in our bijective study, by pulling apart elementary pieces that compose an element of $\mathbf{L} \mathbf{M}_{g, k}$. Our presentation resembles that of [37], the two differences being that our maps are rooted, which allows us to get rid of the symmetry factors, and, more importantly, the vertices are labeled. In the following, it will often be implicit that $(g, k) \notin\{(0,1),(0,2)\}$, which need special treatment.

\subsection{Map reductions}

We let $\mathbf{M}_{g, k}$ be the set of rooted maps of genus $g$ with $k$ indexed faces. Elements of $\mathbf{M}_{g, k}$ for fixed $k$ but with a large number of vertices, show a dendritic pattern branching on a graph with a simple structure. In order to study this in a precise way, we are going to perform several reductions on the maps. We let $\mathbf{M}_{g, k}^{\geq 2}$ and $\mathbf{M}_{g, k}^{\geq 3}$ be the subsets of $\mathbf{M}_{g, k}$ consisting of the maps in which all vertices have degree at least 2 , resp. at least 3 . It is easy to see that $\mathbf{M}_{g, k}^{\geq 2}$ is an infinite set (take a simple loop with an arbitrary number of edges). On the other hand, $\mathbf{M}_{g, k}^{\geq 3}$ is finite according to the combination of Euler's formula

$$
|V|-|\mathbf{E}|+k=2-2 g
$$

and the fact that

$$
2|\mathbf{E}|=\sum_{v \in V} \operatorname{deg} v \geq 3|V|,
$$

which entails $|\mathbf{E}| \leq 3 k+6 g-6$. Note that equality holds if and only if every vertex has degree 3 exactly. We let $\mathbf{M}_{g, k}^{3}$ be the subset of $\mathbf{M}_{g, k}^{\geq 3}$ for which this extra constraint is satisfied. According to these definitions, the set $\mathbf{M}_{0,2}^{\geq 3}$ should be empty. We make an exception by letting $\mathbf{M}_{0,2}^{3}=\mathbf{M}_{0,2}^{\geq 3}$ consist in the two loop maps made of a rooted loop with an origin, bounding two faces with degree 1 (there are two such maps according to whether the face indexed 1 stands to the left or to the right of the root edge). Elements of $\mathbf{M}_{g, k}^{\geq 3}$ will usually be denoted by the letter $\mathfrak{m}$, and their root by $\mathfrak{e}_{*}$.

There are natural projection maps

$$
\mathbf{M}_{g, k} \longrightarrow \mathbf{M}_{g, k}^{\geq 2} \quad, \quad \mathbf{M}_{g, k} \longrightarrow \mathbf{M}_{g, k}^{\geq 3},
$$

which roughly consist in taking the largest subgraph belonging to $\mathbf{M}_{g, k}^{\geq 2}$ (resp. $\mathbf{M}_{g, k}^{\geq 3}$ ), for some appropriate definition of subgraphs, where merging edges are allowed. More precisely, starting from (a graphical realization of) $\mathbf{m} \in \mathbf{M}_{g, k}$, we delete one by one the edges incident to vertices with degree 1 until no such vertex remains. This operation does not disconnect the graph, nor does it modify its genus or the number of its faces. The outcome is thus a map $\mathbf{m} \geq 2$ with $k$ faces and without vertices of degree 1 , and which does not depend on the order of the edges that were removed. If $\mathbf{m}$ has root $e_{*}$, we naturally root $\mathbf{m}^{\geq 2}$ at the first element of $\varphi_{\mathbf{m}}^{i}(e), i \geq 0$ that belongs to $E\left(\mathbf{m}^{\geq 2}\right)$. This yields an element $\mathbf{m}^{\geq 2} \in \mathbf{M}_{g, k}^{\geq 2}$.

Likewise, starting from $\mathbf{m} \in \mathbf{M}_{g, k}$, we can remove all the vertices of degree 2 in $\mathbf{m}^{\geq 2}$ : we concatenate the half-edges $e_{1}, \ldots, e_{r}$ of a simple chain linking two vertices of $\mathbf{m} \geq 2$ of degrees $\geq 3$, and traversing only degree- 2 vertices, into a single edge $\mathfrak{e}=e_{1} \ldots e_{r}$. We root the resulting graph at the one edge $\mathfrak{e}_{*}=e_{1} \ldots e_{r}$ in which the root of $\mathbf{m}^{\geq 2}$ is involved. This does not modify the genus nor the number of faces of the map, and these merging operations 
commute, and thus yield a well-defined element $\mathbf{m}^{\geq 3}$ of $\mathbf{M}_{g, k}^{\geq 3}$. When $(g, k)=(0,2)$, the map $\mathbf{m} \geq 2$ is a cycle $\left(e_{1}, \ldots, e_{r}\right)$ of oriented edges and their reversals, which we concatenate to form a loop. It is also obvious by definition that $\left(\mathbf{m}^{\geq 2}\right)^{\geq 3}=\mathbf{m}^{\geq 3}$. There are natural inclusions $V\left(\mathbf{m}^{\geq 3}\right) \subseteq V\left(\mathbf{m}^{\geq 2}\right) \subseteq V(\mathbf{m})$ and $E\left(\mathbf{m}^{\geq 2}\right) \subseteq E(\mathbf{m})$. The map $\mathbf{m}^{\geq 3}$ is sometimes called the homotopy type of $\mathbf{m}$. See Figure 5 .

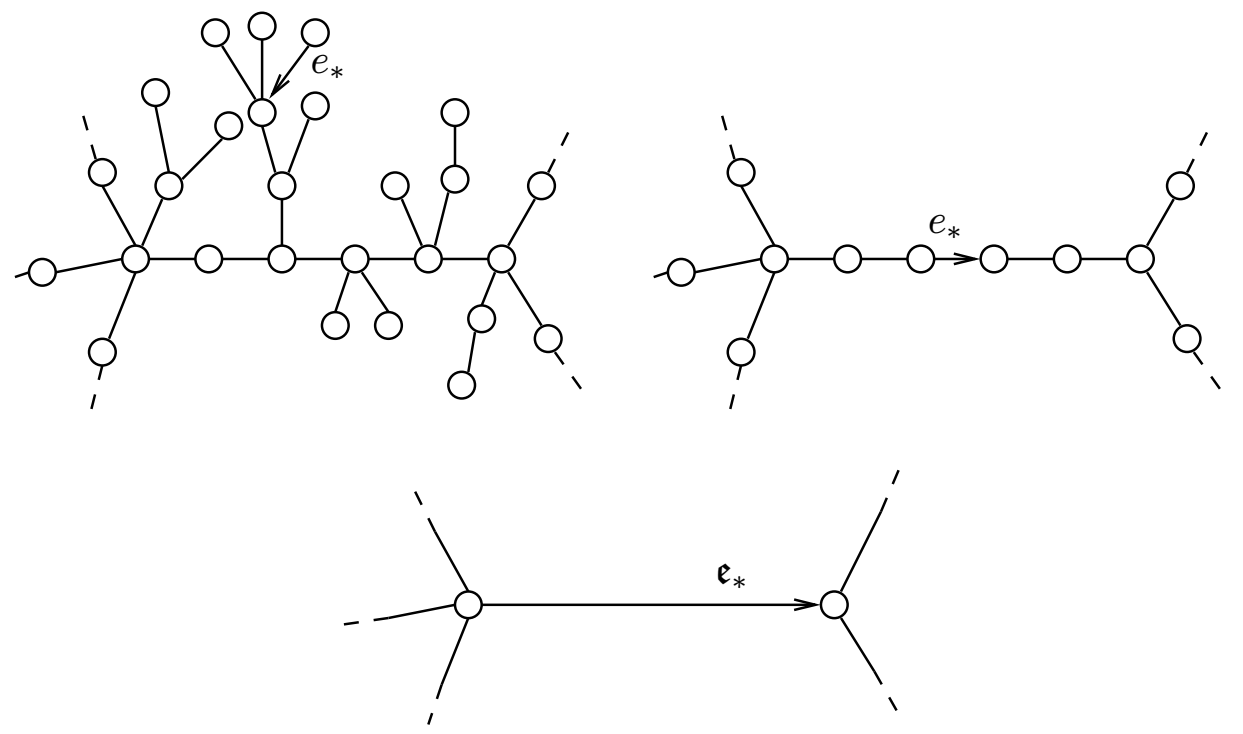

Figure 5. Top-left: a portion of a map $\mathbf{m}$, emphasizing the tree components branching on $\mathbf{m}^{\geq 2}$ depicted on top-right. Bottom: the projection $\mathbf{m}^{\geq 3}$. The picture shows how the various projections are re-rooted depending on the location of the root of $\mathbf{m}$.

\subsection{Some notations for continuous paths}

Certain continuous functions will be crucial to encode maps, so let us introduce some notations. A path is a continuous function $(w(s), 0 \leq s \leq \tau(w))$ taking its values in $\mathbb{R}$, such that $w(0)=0$. The number $\tau(w) \geq 0$ is called its duration, and $\widehat{w}=w(\tau(w))$ its terminal value. We also let $\bar{w}=w(\tau(w)-\cdot)-\widehat{w}$ be the reversed path (beware that this does not mean the same as the reversal of an edge in a graph). We let $\mathcal{W}$ be the set of paths, and make it a Polish space by endowing it with the distance

$$
\operatorname{dist}\left(w, w^{\prime}\right)=\left(\sup _{s \geq 0}\left|w(s \wedge \tau(w))-w^{\prime}\left(s \wedge \tau\left(w^{\prime}\right)\right)\right|\right) \vee\left|\tau(w)-\tau\left(w^{\prime}\right)\right| .
$$

If $w, w^{\prime} \in \mathcal{W}$, we let $w w^{\prime}$ be the concatenation of $w$ and $w^{\prime}$ defined by

$$
w w^{\prime}(s)=\left\{\begin{array}{cl}
w(s) & \text { if } 0 \leq s \leq \tau(w) \\
\widehat{w}+w^{\prime}(s-\tau(w)) & \text { if } \tau(w) \leq s \leq \tau(w)+\tau\left(w^{\prime}\right)=\tau\left(w w^{\prime}\right) .
\end{array}\right.
$$

For $(g, k) \notin\{(0,1),(0,2)\}$ introduce the sets

$$
\mathbf{C}_{g, k}=\left\{\left(\mathfrak{m}, \mathfrak{l}, t^{*}\right): \mathfrak{m} \in \mathbf{M}_{g, k}^{\geq 3}, \mathfrak{l}=\left(\mathfrak{l}^{\mathfrak{e}}, \mathfrak{e} \in E(\mathfrak{m})\right) \in\left(\mathcal{W}^{3}\right)^{E(\mathfrak{m})}, 0 \leq t^{*} \leq \tau^{\mathfrak{e}_{*}}\right\}
$$


endowed with the natural product topology. We make the exceptions $\mathbf{C}_{0,1}=\left\{(c, z) \in \mathcal{W}^{2}\right\}$ and

$$
\mathbf{C}_{0,2}=\left\{\left(\mathfrak{m}, \mathfrak{l}, t^{*}\right): \mathfrak{m} \in \mathbf{M}_{0,2}^{\geq 3}, \mathfrak{l}=\left((c, z), \mathfrak{e}^{\mathfrak{e}_{*}}, \mathfrak{l}^{\overline{\mathfrak{e}}_{*}}\right) \in \mathcal{W}^{2} \times \mathcal{W}^{3} \times \mathcal{W}^{3}, 0 \leq t^{*} \leq \tau(c)\right\} .
$$

For $(g, k) \notin\{(0,1),(0,2)\}$ the generic notations are

$$
\mathfrak{l}^{\mathfrak{e}}=\left(w^{\mathfrak{e}}, c^{\mathfrak{e}}, z^{\mathfrak{e}}\right), \quad r_{\mathfrak{e}}=\tau\left(w^{\mathfrak{e}}\right), \quad \tau^{\mathfrak{e}}=\tau\left(c^{\mathfrak{e}}\right), \quad \tau_{\mathfrak{l}}=\sum_{\mathfrak{e} \in E(\mathfrak{m})} \tau^{\mathfrak{e}} .
$$

For $g=0$ and $k=1,2$ we let $\tau=\tau(c)$, for $k=2$ we also let $\tau_{\mathfrak{l}}=\tau+\tau^{\mathfrak{e}_{*}}+\tau^{\overline{\mathfrak{e}}_{*}}$. Our next task is to bijectively encode elements $\mathbf{m}$ of $\mathbf{L M}_{g, k}$ with certain elements of $\mathbf{C}_{g, k}$, whose first component is the homotopy type of $\mathbf{m}$.

\subsection{Level sets of $\mathbf{M}_{g, k} \rightarrow \mathbf{M}_{g, k}^{\geq 3}$}

Let us see how to get back from the projection $\mathfrak{m}=\mathbf{m}^{\geq 3}$ to the initial map. First of all, every $\mathfrak{e} \in E(\mathfrak{m})$ must be split into a certain number of edges $e_{1} \ldots e_{r_{\mathfrak{e}}}$, with $r_{\mathfrak{e}} \geq 1$, to yield the element $\mathbf{m}^{\geq 2}$, and obviously $r_{\mathfrak{e}}=r_{\overline{\mathfrak{e}}}:=r_{\mathbf{e}}$ (where $\left.\mathbf{e}=\{\mathfrak{e}, \overline{\mathfrak{e}}\}\right)$ so what is needed to do this are positive integers $\left(r_{\mathbf{e}}, \mathbf{e} \in \mathbf{E}(\mathfrak{m})\right)$.

Then, consider elements $e \in E(\mathbf{m})$ such that the first element of $\varphi_{\mathbf{m}}^{i}(e), i \geq 0$ belonging to $E\left(\mathbf{m}^{\geq 2}\right)$ is one of the edges $e_{1}, \ldots, e_{r_{\mathbf{e}}}$. These edges form an interval, and we let $\mathfrak{e}(0)$ be the first element in this interval, and label the others as $\mathfrak{e}(i)=\varphi_{\mathbf{m}}^{i}(\mathfrak{e}(0)), 0 \leq i \leq \tau^{\mathfrak{e}}-1$. With this labeling, we see that every half-edge of $\mathbf{m}$ is of the form $\mathfrak{e}(i)$ for a unique $\mathfrak{e} \in \mathfrak{m}$. Moreover, the half edges of the intervals $\left(e_{j-1}, e_{j}\right), 1 \leq j \leq r_{\mathbf{e}}$ must form an acyclic graph, i.e. a tree, possibly reduced to a single root vertex (in particular, their set has even cardinality and is stable under edge-reversal). Here, we take the convention that $\left(e_{0}, e_{1}\right)$ are the elements of the list $\mathfrak{e}(0), \mathfrak{e}(1), \ldots$ that precede $e_{1}$ strictly. Otherwise said, we can interpret $\mathfrak{e}(0), \ldots, \mathfrak{e}\left(\tau^{\mathfrak{e}}-1\right)$ as a forest of $r_{\mathbf{e}}$ trees, with roots bound together by the floor half-edges $e_{1}, \ldots, e_{r_{\mathbf{e}}}$. If $\mathfrak{e}_{*}$ is the root edge of $\mathbf{m}^{\geq 3}$, then it deserves special attention. Indeed, in this case, one of the edges of the interval $\left[\mathfrak{e}_{*}(0), \mathfrak{e}_{*}\left(\tau^{\mathfrak{e}_{*}}-1\right)\right]$ must be distinguished as the initial root edge of $\mathbf{m}$, which amounts to distinguishing an integer $t^{*} \in\left[0, \tau^{\mathfrak{e}_{*}}\right)$.

The contour function associated with this forest is defined by

$$
c^{\mathfrak{e}}(i)=d_{\mathbf{m}}\left(\mathfrak{e}(i)^{-}, V\left(\mathbf{m}^{\geq 2}\right)\right)-\underline{c}^{\mathfrak{e}}(i), \quad 0 \leq i \leq \tau^{\mathfrak{e}},
$$

where

$$
\underline{c}^{\mathfrak{e}}(i)=\left|\left\{j: e_{j} \in[\mathfrak{e}(0), \mathfrak{e}(i)]\right\}\right|=-\inf _{0 \leq j \leq i} c^{\mathfrak{e}}(j), \quad 0 \leq i \leq \tau^{\mathfrak{e}}-1,
$$

and $\underline{c}^{\mathfrak{e}}\left(\tau^{\mathfrak{e}}\right)=r_{\mathbf{e}}$, is the number of trees in the forest already explored before time $i$. We extend $c^{\mathfrak{e}}, \underline{c}^{\mathfrak{e}}$ into continuous functions ( $\operatorname{still}$ denoted by $c^{\mathfrak{e}}, \underline{\underline{e}}^{\mathfrak{e}}$ ) by linear interpolation between values taken at integers. The result is a concatenation of $r_{\mathbf{e}}$ Dyck paths ${ }^{(3)}$ encoding the trees of the forest, and achieving a new negative infimum when a floor edge of the forest is traversed (note that the terminal value $-r_{\mathbf{e}}$ of $c^{\mathfrak{e}}$ is attained for the first time at the final time $\tau^{\mathfrak{e}}$ ). It is an easy and well-known fact that the contour function entirely determines the forest. In this way we have shown

(3) Here we will call Dyck path a piecewise linear element $c \in \mathcal{W}$ starting at 0 , with slope \pm 1 between integer times, and which attains the value -1 for the first time at $\tau(c)$. 


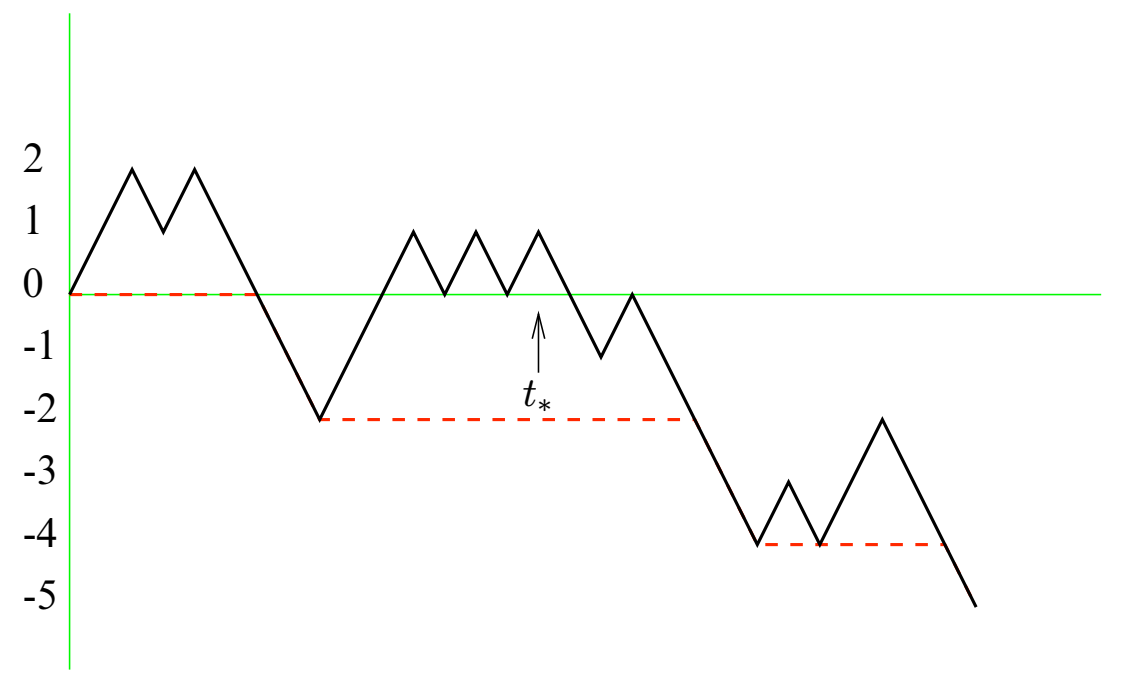

FIGURE 6. The contour process $c^{\mathfrak{e}_{*}}$ associated with the edge $\mathfrak{e}_{*}$ in the reconstruction of the map $\mathbf{m}$ from $\mathbf{m}^{\geq 3}$ as depicted in Figure 5. The process $-\underline{c}^{\mathfrak{e}_{*}}$ is drawn in thick dashed lines. The location of the root of $\mathbf{m}$ is distinguished by the time $t^{*}$.

Lemma 4. - Assume $(g, k) \notin\{(0,1),(0,2)\}$. An element $\mathbf{m} \in \mathbf{M}_{g, k}$ is unambiguously described by its projection $\mathfrak{m}=\mathbf{m}^{\geq 3}$, a family $\left(c^{\mathfrak{e}}, \mathfrak{e} \in E(\mathfrak{m})\right) \in \mathcal{W}^{E(\mathfrak{m})}$ such that $c^{\mathfrak{e}}$ is the concatenation of $r_{\mathbf{e}}$ Dyck paths, and an integer $t^{*} \in\left[0, \tau^{\mathfrak{e}_{*}}\right)$.

See Figure 6 for an illustration. The cases $(g, k)=(0,1)$ or $(0,2)$ are special and require a little discussion. An element of $\mathbf{M}_{0,1}$ is simply a rooted planar tree with at least one edge, and is described by a single Dyck path $c$ with duration $\tau(c)>1$. When $(g, k)=(0,2)$, the construction of $\mathbf{m}^{\geq 3}$ from an element $\mathbf{m} \in \mathbf{M}_{0,2}$ shows that the root vertex of $\mathbf{m}^{\geq 3}$ can be any of the vertices of the loop constituting $\mathbf{m}^{\geq 2}$. This difficulty does not appear for other values of $(g, k)$, since the root edge of $\mathbf{m}^{\geq 3}$ has an unambiguous origin in the original map $\mathbf{m}$. While this does not affect the final outcome considered as a map, there are several nonequivalent ways to obtain the same result. To lift the ambiguity, we use the extra convention that the root vertex in $\mathbf{m}^{\geq 3}$ is $e^{-}$, where $e$ is the first element of $E\left(\mathbf{m}^{\geq 2}\right)$ in the sequence $\left(\varphi_{\mathbf{m}}^{i}\left(e_{*}\right), i \geq 0\right)$. Otherwise said, we single out the tree component that contains the root and branching on the unique cycle of $\mathbf{m}$, by saying that its root vertex must be the root vertex of $\mathbf{m} \geq 3$. The other tree components are obtained by taking concatenations of Dyck paths so that an element $\mathbf{m} \in \mathbf{M}_{0,2}$ is unambiguously described by the element $\mathbf{m}^{\geq 3}$, a Dyck path $c$, an integer time in $[0, \tau(c))$ and two paths $c^{\mathfrak{e}_{*}}, c^{\overline{\mathfrak{e}}_{*}}$, the first being a concatenation of $r_{\mathfrak{e}_{*}}-1$ Dyck paths, and the second the concatenation of $r_{\mathfrak{e}_{*}}$ Dyck paths.

\subsection{Labeling the maps}

In order to complete the description of elements of $\mathbf{L M}_{g, k}$, we need to incorporate the labeling function [1]. We can use the origin of the root edge $\mathfrak{e}_{*}$ of $\mathfrak{m}=\mathbf{m}^{\geq 3}$ as a reference for $[\mathbf{l}]$, i.e. we can choose the element $\mathbf{l} \in[\mathbf{l}]$ such that $\mathbf{l}\left(\mathfrak{e}_{*}^{-}\right)=0$. This convention will be 
in force from this section onwards. It is then natural to introduce a labeling function $l^{\mathfrak{e}}$ for every $\mathfrak{e} \in E(\mathfrak{m})$, defined by

$$
l_{i}^{\mathfrak{e}}=\mathbf{l}(\mathfrak{e}(i))-\mathbf{l}(\mathfrak{e}(0)), \quad 0 \leq i<\tau^{\mathfrak{e}},
$$

and $l_{\tau^{\mathfrak{e}}}^{\mathfrak{e}}=\mathbf{l}\left(\mathfrak{e}\left(\tau^{\mathfrak{e}}-1\right)^{+}\right)-\mathbf{l}(\mathfrak{e}(0))$, where the notation $\mathfrak{e}(0), \mathfrak{e}(1), \ldots$ is as above the list of edges of the trees branching on $\mathfrak{e}$. We interpolate linearly between integer times, in order to define a continuous piecewise linear function with slope in $\{-1,0,1\}$, starting at 0 and with same duration as $c^{\mathfrak{e}}$. Another process that will be of crucial importance is the process

$$
l_{i}=\mathbf{l}\left(\varphi_{\mathbf{m}}^{i}\left(\mathfrak{e}_{*}(0)\right)\right) \quad 0 \leq i \leq \operatorname{deg}_{\mathbf{m}}\left(f_{*}\right)-1,
$$

where $f_{*}$ is the face of $\mathbf{m}$ incident to $e_{*}$, and $l_{\operatorname{deg}_{\mathbf{m}}\left(f_{*}\right)}=0$. As usual, it is turned into an element of $\mathcal{W}$ by linear interpolation. In the case where $\mathbf{m}$ has only one face, we see that $l$ provides a natural exploration of the labels of all vertices of $\mathbf{m}$ in the fatgraph face order. It is easily checked that $l$ is the concatenation

$$
l=l^{\mathfrak{e}_{*}} l^{\varphi_{\mathfrak{m}}\left(\mathfrak{e}_{*}\right)} \ldots l^{\varphi_{\mathfrak{m}}^{\operatorname{deg}_{\mathfrak{m}}\left(\mathfrak{f}_{*}\right)-1}\left(\mathfrak{e}_{*}\right)},
$$

where $\mathfrak{f}_{*}$ is the face of $\mathfrak{m}$ incident to $\mathfrak{e}_{*}$. In order to study scaling limits, we will still need to decompose $l^{\mathfrak{e}}$ into two more elementary pieces, which is the purpose of the two next sections.

3.4.1. Walk networks. - A walk is an element $w \in \mathcal{W}$ taking integer values on $\mathbb{Z}_{+}$, interpolating linearly between these values, and satisfying $|w(i)-w(i-1)| \leq 1$ for $1 \leq i \leq r:=\tau(w)$ (where it is assumed that $r \geq 1$ ). Let $\mathbf{W}$ be the set of walks. A walk from 0 to $x \in \mathbb{Z}$ is a walk with final value $\widehat{w}=x$, their set is written $\mathbf{W}_{0 \rightarrow x}$.

Let $\mathfrak{m} \in \mathbf{M}_{g, k}^{\geq 3}$ for some $g, k \geq 0$. We let $\ell(V)$ and $\ell(E)$ be the spaces of functions $V(\mathfrak{m}) \rightarrow \mathbb{R}$ and $E(\mathfrak{m}) \rightarrow \mathbb{R}$ respectively. We also let $\ell_{0}(V)$ be those functions 1 of $\ell(V)$ with $\mathbf{l}\left(\mathfrak{e}_{*}^{-}\right)=0$. A function $\theta \in \ell(E)$ is antisymmetric if $\theta(\mathfrak{e})=-\theta(\overline{\mathfrak{e}})$ for every $\mathfrak{e}$, and we let $\ell_{-}(E)$ be the space of antisymmetric functions. For $\mathbf{l} \in \ell(V)$, we let $\nabla \mathbf{l} \in \ell_{-}(E)$ be defined by $\nabla \mathbf{l}(\mathfrak{e})=\mathbf{l}\left(\mathfrak{e}^{+}\right)-\mathbf{l}\left(\mathfrak{e}^{-}\right)$. As $\mathfrak{m}$ is connected, the kernel of the linear map $\nabla$ is the space of constant functions, so the restriction of $\nabla$ to $\ell_{0}(V)$ is an isomorphism of vector spaces. A function $\theta$ in the image $\nabla_{V}$ of $\nabla$ is called a potential.

A walk network on $\mathfrak{m}$ is a collection $\left(w^{\mathfrak{e}}, \mathfrak{e} \in E(\mathbf{m})\right) \in \mathbf{W}^{E(\mathbf{m})}$ such that

$$
w^{\overline{\mathfrak{e}}}(t)=w^{\mathfrak{e}}\left(r_{\mathbf{e}}-t\right)-\widehat{w}^{\mathfrak{e}}, \quad 0 \leq t \leq r_{\mathbf{e}}=\tau\left(w^{\mathfrak{e}}\right)=\tau\left(w^{\overline{\mathfrak{e}}}\right),
$$

and such that the antisymmetric function $\theta=\widehat{w}=\left(\widehat{w}^{\mathfrak{e}}, \mathfrak{e} \in E(\mathfrak{m})\right)$ is a potential ${ }^{(4)}$. We let $\mathbf{W N}_{\mathfrak{m}}$ be the set of walk networks on $\mathfrak{m}$. If we are given an antisymmetric function $\theta \in \ell_{-}(E)$ assuming integers values, we let $\mathbf{W N}_{\mathfrak{m}}(\theta)$ be the set of walk networks $\left(w^{\mathfrak{e}}, \mathfrak{e} \in E(\mathfrak{m})\right) \in \mathbf{W N}_{\mathfrak{m}}$ such that $\widehat{w}=\theta$.

If $(\mathbf{m},[\mathbf{l}]) \in \mathbf{L M}_{g, k}$, and using the notations of Section 3.3, we set, for every $\mathfrak{e} \in E(\mathfrak{m})$ of the form $\mathfrak{e}=e_{1} \ldots e_{r_{\mathbf{e}}}$,

$$
w^{\mathfrak{e}}(i)=\mathbf{l}\left(e_{i+1}^{-}\right)-\mathbf{l}\left(e_{1}^{-}\right), \quad 0 \leq i \leq r_{\mathbf{e}}-1,
$$

and $w^{\mathfrak{e}}\left(r_{\mathbf{e}}\right)=\mathbf{l}\left(e_{r_{\mathbf{e}}}^{+}\right)-\mathbf{l}\left(e_{1}^{-}\right)$. This defines a walk with duration $r_{\mathbf{e}}$. Moreover, the family $\left(w^{\mathfrak{e}}, \mathfrak{e} \in E(\mathfrak{m})\right)$ is a walk network. The reversal property $w^{\overline{\mathfrak{e}}}(\cdot)=w^{\mathfrak{e}}\left(r_{\mathbf{e}}-\cdot\right)-\widehat{w}^{\mathfrak{e}}$ is clear since $\overline{\mathfrak{e}}=\bar{e}_{r_{\mathbf{e}}} \ldots \bar{e}_{1}$, and by definition the function $\left(\widehat{w}^{\mathfrak{e}}, \mathfrak{e} \in E(\mathfrak{m})\right)$ is $\left.\nabla \mathbf{l}\right|_{V(\mathfrak{m})}$.

(4) Alternatively, walk networks on $\mathfrak{m}$ are in correspondence with labeled maps $\mathbf{m} \in \mathbf{M}_{g, k}^{\geq 2}$ such that $\mathbf{m} \geq 3=\mathfrak{m}$. 
3.4.2. Discrete snakes. - Finally, we say that the pair $(c, z) \in \mathcal{W}^{2}$ is a discrete snake on $r \geq 1$ tree components if

- $c$ is a concatenation of $r$ Dyck paths,

- $z$ is taking integer values on $\mathbb{Z}_{+}$, interpolating linearly between these values, with integer steps in $\{-1,0,1\}$, satisfies $\tau(z)=\tau(c)$, and $z(i)=z(j)$ whenever $c(i)=c(j)=\min _{i \wedge j \leq k \leq i \vee j} c(k)$ (which means that $i$ and $j$ are times where the same vertex of the forest encoded by $c$ is explored),

- moreover, $z(i)=0$ whenever $i$ is an integer time at which $c$ achieves a new minimum (this means that $i$ is a time at which a new tree component is explored, and the label of the root is fixed to be 0 ).

In words, a discrete snake is the process of heights and labels when exploring an integerlabeled forest in contour order, where the labels differ by at most 1 in absolute value between adjacent vertices, and the labels of the forest's roots is 0 . We let $\mathbf{S}_{r}$ be the set of discrete snakes on $r$ tree components.

If $(\mathbf{m},[\mathbf{l}]) \in \mathbf{L M}_{g, k}$ we set, whenever $0 \leq i \leq \tau^{\mathfrak{e}}$ is such that $\mathfrak{e}(i) \in\left(e_{m-1}, e_{m}\right]$ (recall the notations of Section 3.3),

$$
z^{\mathfrak{e}}(i)=\mathbf{l}(\mathfrak{e}(i))-\mathbf{l}\left(e_{m}\right),
$$

and we interpolate linearly between integer times. The pair $\left(c^{\mathfrak{e}}, z^{\mathfrak{e}}\right)$ is then the discrete snake associated with the forest branching on the edge $\mathfrak{e}$, where the labels in each tree component have been shifted so that the roots have labels 0 . With the data $\left(w^{\mathfrak{e}}, c^{\mathfrak{e}}, z^{\mathfrak{e}}\right)$ defined so far, the label function $l^{\mathfrak{e}}$ introduced at the beginning of the subsection is recovered as

$$
l^{\mathfrak{e}}(i)=z^{\mathfrak{e}}(i)+w^{\mathfrak{e}}\left(\underline{c}^{\mathfrak{e}}(i)\right) .
$$

We also use (12) to define the function $l^{\mathfrak{e}}$ out of any $\left(\mathfrak{m}, \mathfrak{l}, t^{*}\right) \in \mathbf{C}_{g, k}$, where $\underline{c}^{\mathfrak{e}}(t)=-\inf _{0 \leq s \leq t} c^{\mathfrak{e}}(s)$ for $0 \leq s \leq \tau^{\mathfrak{e}}$, and define the function $l$ as in (11).

Summing up the study of this section, we obtain

Proposition 1. - (i) Let $(g, k) \notin\{(0,1),(0,2)\}$. Then $\mathbf{L M}_{g, k}$ is in bijection with the subset $\mathbf{C}_{g, k}^{\text {map }} \subset \mathbf{C}_{g, k}$ of elements $\left(\mathfrak{m}, \mathfrak{l}, t^{*}\right)$ such that

1. $\left(w^{\mathfrak{e}}, \mathfrak{e} \in E(\mathfrak{m})\right) \in \mathbf{W N}_{\mathfrak{m}}$,

2. $\left(c^{\mathfrak{e}}, z^{\mathfrak{e}}\right) \in \mathbf{S}_{r_{\mathfrak{e}}}$ for every $\mathfrak{e} \in E(\mathfrak{m})$, and

3. $t^{*}$ is $n$ integer in $\left[0, \tau\left(c^{\mathfrak{e}_{*}}\right)\right)$.

(ii) $\mathbf{L} \mathbf{M}_{0,1}$ is in bijection with the set $\mathbf{C}_{0,1}^{\text {map }}=\left\{(c, z) \in \mathbf{S}_{1}: \tau(c)>1\right\}$.

(iii) $\mathbf{L M}_{0,2}$ is in bijection with the subset $\mathbf{C}_{0,2}^{\operatorname{map}} \subset \mathbf{C}_{0,2}$ of elements $\left(\mathfrak{m}, \mathfrak{l}, t^{*}\right)$ with

1. $w^{\mathfrak{e}_{*}} \in \mathbf{W}_{0 \rightarrow 0}$ and $w^{\overline{\mathfrak{e}}_{*}}=\bar{w}^{\mathfrak{e}_{*}}$,

2. $(c, z) \in \mathbf{S}_{1}$ and $t^{*} \in[0, \tau(c))$ is an integer,

3. $\left(c^{\mathfrak{e}_{*}}, z^{\mathfrak{e}_{*}}\right) \in \mathbf{S}_{r_{\mathfrak{e}_{*}}-1}$ and $\left(c^{\overline{\mathfrak{e}}_{*}}, z^{\overline{\mathfrak{e}}_{*}}\right) \in \mathbf{S}_{r_{\mathfrak{e}_{*}}}$. 


\section{Discrete and continuous Boltzmann measures}

We now explore natural measures and probability distributions on tessellated genus- $g$ maps, and their continuous counterparts.

We define the measure $\mathbf{Q}_{g, k}$ on the set $\mathbf{Q}_{g, k}$ as the $\sigma$-finite measure assigning mass $12^{-|F(\mathbf{q})|}$ to every element $(\mathbf{q}, \mathbf{x}, D)$. Otherwise said, if we recall the notations of Section 1.4 , one has

$$
\mathrm{Q}_{g, k}(\mathrm{~d}(\mathbf{q}, \mathbf{x}, D))=\mathrm{Q}_{g}(\mathrm{~d} \mathbf{q})\left(V_{\mathbf{q}} \mu_{\mathbf{q}}\right)^{\otimes k}(\mathrm{~d} \mathbf{x}) \#_{\mathcal{D}(\mathbf{q}, \mathbf{x})}(\mathrm{d} D),
$$

where $\#_{\mathcal{D}(\mathbf{q}, \mathbf{x})}(\mathrm{d} D)$ is the counting measure on the (possibly empty) set of delays in $\mathbf{q}$ between sources $\mathbf{x}$. Up to Section 4.4 we assume $(g, k) \notin\{(0,1),(0,2)\}$.

According to Theorem 4 and Corollary 1 , the push-forward of $\mathrm{Q}_{g, k}$ by the mapping $\Psi_{g, k}$ is the measure on the set $\mathbf{L} \mathbf{M}_{g, k}$ that assigns mass $2 \cdot 12^{-|\mathbf{E}(\mathbf{m})|}$ to any element $(\mathbf{m},[\mathbf{l}])$. Using the considerations of Section 3, we see that this measure can in turn be obtained out of measures on the more elementary pieces that compose a labeled map (walk networks and snakes). More precisely, consider the bijective mapping $\Xi_{g, k}: \mathbf{L M}_{g, k} \rightarrow \mathbf{C}_{g, k}^{\text {map }}$ that is described in Proposition 1. Define

$$
\widetilde{\Psi}_{g, k}:=\Xi_{g, k} \circ \Psi_{g, k} \quad: \quad \mathbf{Q}_{g, k} \rightarrow \mathbf{C}_{g, k}^{\operatorname{map}} .
$$

Then the image measure $\mathrm{LM}_{g, k}$ of $\mathrm{Q}_{g, k}$ by $\widetilde{\Psi}_{g, k}$ assigns weight $2 \cdot 12^{-\tau_{\mathfrak{l}} / 2}$ to $\left(\mathfrak{m}, \mathfrak{l}, t^{*}\right) \in \mathbf{C}_{g, k}^{\text {map }}$, as one checks that $\tau_{\mathfrak{l}} / 2=|\mathbf{E}(\mathbf{m})|=|F(\mathbf{q})|=V_{\mathbf{q}}-\chi(g)$ whenever $(\mathbf{q}, \mathbf{x}, D),(\mathbf{m},[\mathbf{l}]),\left(\mathfrak{m}, \mathfrak{l}, t^{*}\right)$ are associated by our bijections. We rewrite this measure in the form

$$
\mathrm{LM}_{g, k}=2 \sum_{\mathfrak{m} \in \mathbf{M}_{g, k}^{\geq 3}} \mathrm{LM}_{\mathfrak{m}}
$$

where

$$
\mathrm{LM}_{\mathfrak{m}}=\delta_{\{\mathfrak{m}\}} \mathrm{WN}_{\mathfrak{m}}\left(\mathrm{d}\left(w^{\mathfrak{e}}\right)_{\mathfrak{e} \in E(\mathfrak{m})}\right) \bigotimes_{\mathfrak{e} \in E(\mathfrak{m})} \mathrm{DS}_{\tau\left(w^{\mathfrak{e}}\right)}\left(\mathrm{d}\left(c^{\mathfrak{e}}, z^{\mathfrak{e}}\right)\right) \#_{\tau\left(c^{\mathfrak{e} *}\right)}\left(\mathrm{d} t^{*}\right)
$$

and

- $\mathbf{W N}_{\mathfrak{m}}$ is the measure assigning weight $3^{-\sum_{\mathbf{e} \in \mathbf{E}(\mathfrak{m})} r_{\mathbf{e}}}$ to a walk network $\left(w^{\mathfrak{e}}\right) \in \mathbf{W N}_{\mathfrak{m}}$,

- $\mathrm{DS}_{r}$ is the measure assigning weight $2^{-\tau(c)} 3^{-(\tau(c)-r) / 2}$ to an element $(c, z) \in \mathbf{S}_{r}$,

- lastly, $\#_{\tau\left(c^{\mathfrak{e}_{*}}\right)}$ is the counting measure on $\left\{0, \ldots, \tau\left(c^{\mathfrak{e}_{*}}\right)-1\right\}$.

Individually, these measures are $\sigma$-finite measures, which are finite except for $\mathrm{WN}_{\mathfrak{m}}$. The measure $\mathrm{DS}_{r}$ is a probability measure, under which the $r$ trees involved in the forest are independent Galton-Watson trees with geometric $(1 / 2)$ offspring distribution, and with uniformly distributed labels among the allowed ones, conditionally on the forest. To see this, recall that $(c, z) \in \mathbf{S}_{r}$ encodes a forest with $r$ tree components, and $\tau(c)$ oriented edges (counting the $r$ floor edges between tree components). A component of this forest, which is a labeled tree with $m^{\prime}$ edges say, contributes a probability factor $2^{-2 m^{\prime}-1} 3^{-m^{\prime}}$ to the weight $2^{-\tau(c)} 3^{-(\tau(c)-r) / 2}$. Also, the measure $\mathrm{DS}_{r}(\mathrm{~d}(c, z)) \#_{\tau(c)}\left(\mathrm{d} t^{*}\right)$ is an infinite measure.

\subsection{Walk networks}

For simplicity we let in this section $V=V(\mathfrak{m}), E=E(\mathfrak{m}), \mathbf{E}=\mathbf{E}(\mathfrak{m})$ for some $\mathfrak{m} \in \mathbf{M}_{g, k}^{\geq 3}$. We let $\widehat{W N}_{\mathfrak{m}}$ be the image measure of $\operatorname{WN}_{\mathfrak{m}} \operatorname{under}\left(w^{\mathfrak{e}}, \mathfrak{e} \in E\right) \mapsto\left(\left(r_{\mathbf{e}}, \mathbf{e} \in \mathbf{E}\right),\left(\widehat{w}^{\mathfrak{e}}, \mathfrak{e} \in E\right)\right)$. 
4.1.1. Gaussian laws on potentials. - Fix $\mathfrak{m} \in \mathbf{M}_{g, k}$, and let $v_{*}=\mathfrak{e}_{*}^{-}$be the root vertex. Recall the notations of Section 3.4. We endow $\ell_{0}(V)$ with the scalar product $\left(\mathbf{x}, \mathbf{x}^{\prime}\right)=\sum_{v \neq v_{*}} x_{v} x_{v}^{\prime}$, and endow the vector space $\nabla_{V}:=\nabla \ell_{0}(V) \subset \ell_{-}(E)$ of potentials on $\mathfrak{m}$ with the scalar product

$$
\left\langle\nabla \mathbf{x}, \nabla \mathbf{x}^{\prime}\right\rangle=\left(\mathbf{x}, \mathbf{x}^{\prime}\right) .
$$

This space is isometric to $\ell_{0}(V)$, and of dimension $|V|-1=\operatorname{dim} \ell_{0}(V)$. We let $\lambda_{\nabla}$ be the measure on $\nabla_{V}$ which is the image of $\prod_{v \in V \backslash\left\{v_{*}\right\}} \mathrm{d} y_{v}$ by $\nabla: \ell_{0}(V) \rightarrow \ell_{-}(E)$, and call it the Lebesgue measure on $\nabla_{V}$ : it assigns mass 1 to the unit cube delimited by the vectors of the orthonormal basis $\left(\nabla \mathbb{1}_{v}, v \neq v_{*}\right)$. For $\left(r_{\mathbf{e}}, \mathbf{e} \in \mathbf{E}\right)$ a family of positive weights, we also let

$$
\mathcal{E}_{\mathbf{r}}(\mathbf{x})=\sum_{\mathbf{e} \in \mathbf{E}} x_{\mathbf{e}}^{2} / r_{\mathbf{e}}
$$

which defines a positive definite quadratic form on $\nabla_{V}$. The Gaussian distribution on $\nabla_{V}$ associated with $\mathcal{E}_{\mathbf{r}}$ is the measure

$$
\mathcal{N}_{\mathbf{r}}^{\mathfrak{m}}(\mathrm{d} \mathbf{x})=\frac{1}{(2 \pi)^{\frac{|V|-1}{2}}} \exp \left(-\frac{\mathcal{E}_{\mathbf{r}}(\mathbf{x})}{2}\right) \sqrt{\operatorname{det} \mathcal{E}_{\mathbf{r}}} \lambda_{\nabla}(\mathrm{d} \mathbf{x})
$$

which is a probability distribution. One should be careful that $\operatorname{det} \mathcal{E}_{\mathbf{r}}$ is the determinant of the quadratic form defined on $\left(\nabla_{V},\langle\cdot, \cdot\rangle\right)$ and not the whole space $\ell_{-}(E)$.

Lemma 5. - Let $\mathrm{ST}(\mathfrak{m})$ denote the set of spanning trees of $\mathfrak{m}$. Then, one has

$$
\operatorname{det} \mathcal{E}_{\mathbf{r}}=\sum_{\mathfrak{a} \in \operatorname{ST}(\mathfrak{m})} \prod_{\mathbf{e} \in \mathbf{E}(\mathfrak{a})}\left(r_{\mathbf{e}}\right)^{-1}
$$

Otherwise said, this determinant is a partition function for spanning trees on $\mathfrak{m}$ with weight $1 / r_{\mathbf{e}}$ for the edge $\mathbf{e}$.

Proof. - In the orthonormal basis $\left(\nabla \mathbb{1}_{\{v\}}, v \neq v_{*}\right)$ of $\left(\nabla_{V},\langle\cdot, \cdot\rangle\right)$, it is easy to check that the matrix $\left(m_{u v}, u, v \in V \backslash\left\{v_{*}\right\}\right)$ of the quadratic form $\mathcal{E}_{\mathbf{r}}$ is given by $m_{v v}=\sum_{\mathfrak{e}: \mathfrak{e}^{-}=v} 1 / r_{\mathfrak{e}}$ and $m_{u v}=-\sum_{\mathfrak{e}: \mathfrak{e}^{-}=u, \mathfrak{e}^{+}=v} 1 / r_{\mathfrak{e}}$. Otherwise said, this is a minor (excluding row and column corresponding to $v_{*}$ ) of the weighted Laplacian matrix on $V$ with weight $1 / r_{\mathfrak{e}}$ on edge $\mathfrak{e}$, and one concludes by the matrix-tree theorem (and the result does not depend on the root of $\mathfrak{m}$ but only on the map).

The following result will also be useful when estimating integrals with respect to $\lambda_{\nabla}$. For a spanning tree $\mathfrak{a} \in \mathrm{ST}(\mathfrak{m})$, we privilege the orientation of its edges $E_{1 / 2}(\mathfrak{a})$ by letting them point away from the root vertex $v_{*}$. If $\mathbf{y} \in \mathbb{R}^{E_{1 / 2}(\mathfrak{a})}$, we define a function $\theta_{\mathbf{y}}^{\mathfrak{a}} \in \ell_{-}(E)$ extending $\mathbf{y}$ by letting $\theta_{\mathbf{y}}^{\mathfrak{a}}(\overline{\mathfrak{e}})=-y_{\mathfrak{e}}$ for $\mathfrak{e} \in E_{1 / 2}(\mathfrak{a})$ and then

$$
\theta_{\mathbf{y}}^{\mathfrak{a}}(\mathfrak{e})=\sum_{\mathfrak{e}^{\prime}} y_{\mathfrak{e}^{\prime}}
$$

where the sum is over the half-edges $\mathfrak{e}^{\prime} \in E(\mathfrak{a})$ of the unique oriented simple chain going from $\mathfrak{e}^{-}$to $\mathfrak{e}^{+}$. It is easy to see that $\theta_{\mathbf{y}}^{\mathfrak{a}} \in \nabla_{V}$ is a potential, and any potential is recovered from its restriction to $E_{1 / 2}(\mathfrak{a})$ by applying $\theta^{\mathfrak{a}}$. 
Lemma 6. - Let $H: \mathbb{R}^{E} \rightarrow \mathbb{R}_{+}$be measurable. Then for $\mathfrak{a} \in \mathrm{ST}(\mathfrak{m})$,

$$
\int_{\nabla_{V}} \lambda_{\nabla}(\mathrm{d} \mathbf{x}) H(\mathbf{x})=\int_{\mathbb{R}^{E_{1 / 2}(\mathfrak{a})}} \mathrm{d} \mathbf{y} H\left(\theta_{\mathbf{y}}^{\mathfrak{a}}\right) .
$$

Proof. - By definition

$$
\int_{\nabla_{V}} \lambda_{\nabla}(\mathrm{d} \mathbf{x}) H(\mathbf{x})=\int_{\mathbb{R}^{V \backslash\left\{v_{*}\right\}}} \mathrm{d} \mathbf{z} H(\nabla \mathbf{z}) .
$$

The result follows from the change of variables $\mathbf{y}(\mathfrak{e})=\nabla \mathbf{z}(\mathfrak{e}), \mathfrak{e} \in E_{1 / 2}(\mathfrak{a})$, provided we can justify that its Jacobian is 1 . This results from the fact that this change of variables is linear, and that its representative matrix in an appropriate basis is triangular with only 1 's on the diagonal. To see this, perform the search-depth exploration of $\mathfrak{a}$ starting from any half-edge $\mathfrak{e}_{1} \in E(\mathfrak{a})$ with $\mathfrak{e}_{1}^{-}=v_{*}$, and letting, for $i \geq 2, \mathfrak{e}_{i}=\varphi_{\mathfrak{a}}^{m(i)}\left(\mathfrak{e}_{i-1}\right)$ where $m(i)$ is the first integer $j \geq 0$ such that $\left(\varphi_{\mathfrak{a}}^{j}\left(\mathfrak{e}_{i-1}\right)\right)^{+}$is a vertex that does not belong to the set $\left\{v_{*}, \mathfrak{e}_{1}^{+}, \ldots, \mathfrak{e}_{i-1}^{+}\right\}$ of already explored vertices: this is possible as long as $i \leq|V|-1$. This gives a labeling $\mathfrak{e}_{1}, \ldots, \mathfrak{e}_{|V|-1}$ of the half-edges of $\mathfrak{a}$ pointing out from $v_{*}$, such that the source $\mathfrak{e}_{i}^{-}$is always a vertex belonging to the set $\left\{\mathfrak{e}_{j}^{+}: j<i\right\}$, while the target $\mathfrak{e}_{i}^{+}$is a newly explored vertex.

4.1.2. Key lemmas. - Define the quantity

$$
\mathcal{Z}^{\mathfrak{m}}(\mathbf{r})=\sum_{\mathfrak{a} \in \operatorname{ST}(\mathfrak{m})} \prod_{\mathbf{e} \notin \mathbf{E}(\mathfrak{a})} 2 \pi r_{\mathbf{e}}, \quad \mathbf{r} \in \mathbb{R}_{+}^{E},
$$

which is a partition function for the complement of a spanning tree, with weight $r_{\mathbf{e}}$ on edge $\mathbf{e}$, and defines a homogeneous function of degree $|\mathbf{E}|-|V|+1$. We define a measure $\widehat{\mathrm{PN}}_{\mathfrak{m}}$ on $\mathbb{R}_{+}^{\mathbf{E}} \times \nabla_{V}$ by

$$
\widehat{\mathrm{PN}}_{\mathfrak{m}}(\mathrm{d} \mathbf{r}, \mathrm{d} \mathbf{x})=\frac{\mathrm{d} \mathbf{r}}{\sqrt{\mathcal{Z}^{\mathfrak{m}}(\mathbf{r})}} \mathcal{N}_{\mathbf{r}}^{\mathfrak{m}}(\mathrm{d} \mathbf{x}) .
$$

Let $\Delta_{\mathbf{E}}=\left\{\mathbf{u}=\left(u_{\mathbf{e}}, \mathbf{e} \in \mathbf{E}\right) \in \mathbb{R}_{+}^{\mathbf{E}}: \sum_{\mathbf{e} \in \mathbf{E}} u_{\mathbf{e}}=1\right\}$ be the $|\mathbf{E}|$-1-dimensional simplex indexed by $\mathbf{E}$, and $\lambda_{\Delta}$ denote the (scaled) Lebesgue measure on $\Delta_{\mathbf{E}}$, satisfying

$$
\int_{\mathbb{R}_{+}^{\mathbf{E}}} H(\mathbf{r}) \mathrm{d} \mathbf{r}=\int_{\mathbb{R}_{+}} \rho^{|\mathbf{E}|-1} \mathrm{~d} \rho \int_{\Delta_{\mathbf{E}}} \lambda_{\Delta}(\mathrm{d} \mathbf{u}) H(\rho \mathbf{u})
$$

for every integrable $H$. In particular, taking $H(\mathbf{r})=\exp \left(-\sum_{\mathbf{e} \in \mathbf{E}} r_{\mathbf{e}}\right)$, we see that its total mass equals $1 /(|\mathbf{E}|-1)$ !.

We define the measure $\widehat{\mathrm{PN}}_{\mathfrak{m}}^{(\rho)}$ on $\rho \Delta_{\mathbf{E}} \times \nabla_{V}$ as the image measure by $(\mathbf{u}, \mathbf{x}) \mapsto(\rho \mathbf{u}, \mathbf{x})$ of

$$
\rho^{|\mathbf{E}|-1} \frac{\lambda_{\Delta}(\mathrm{d} \mathbf{u})}{\sqrt{\mathcal{Z}^{\mathfrak{m}}(\rho \mathbf{u})}} \mathcal{N}_{\rho \mathbf{u}}^{\mathfrak{m}}(\mathrm{d} \mathbf{x})
$$

hence giving a disintegration of $\widehat{\mathrm{PN}}_{\mathfrak{m}}$ with respect to $\rho(\mathbf{r}):=\sum_{\mathbf{e} \in \mathbf{E}} r_{\mathbf{e}}$.

Lemma 7. - For $\mathbf{u} \in n \Delta_{\mathbf{E}}$, we let $\widetilde{\mathbf{u}}$ be the point of $n \Delta_{\mathbf{E}} \cap \mathbb{Z}^{\mathbf{E}}$ that is closest to $\mathbf{u}$ for the Euclidean metric (taking some arbitrary convention for ties). Then for any bounded function $H: \mathbb{R}_{+}^{\mathbf{E}} \rightarrow \mathbb{R}$,

$$
\left|\frac{1}{n^{|\mathbf{E}|-1}} \sum_{\mathbf{v} \in n \Delta_{\mathbf{E}} \cap \mathbb{Z}^{\mathbf{E}}} H(\mathbf{v})-\int_{\Delta_{\mathbf{E}}} \lambda_{\Delta}(\mathrm{d} \mathbf{u}) H(\widetilde{n \mathbf{u}})\right| \leq(\sup |H|) \varepsilon_{n},
$$

$4^{\text {e }}$ SÉRIE - TOME $42-2009-\mathrm{N}^{\mathrm{o}} 5$ 
where the sequence $\left(\varepsilon_{n}, n \geq 1\right)$ is independent of $H$ and goes to 0 as $n \rightarrow \infty$.

Proof. - To see this, note that

$$
\int_{\Delta_{\mathbf{E}}} \lambda_{\Delta}(\mathrm{d} \mathbf{u}) H(\widetilde{n \mathbf{u}})=\sum_{\mathbf{v} \in n \Delta_{\mathbf{E}} \cap \mathbb{Z}^{\mathbf{E}}} H(\mathbf{v}) \lambda_{\Delta}\left(\left\{\mathbf{u} \in \Delta_{\mathbf{E}}: \widetilde{n \mathbf{u}}=\mathbf{v}\right\}\right) .
$$

Now, as long as $\mathbf{v} \in n \Delta_{\mathbf{E}} \cap \mathbb{Z}^{\mathbf{E}}$ has all its coordinates positive, i.e. does not belong to the boundary $n \partial \Delta_{\mathbf{E}}$ and therefore stands at distance at least 1 from it as it has integer coordinates, the sets $\left\{\mathbf{u} \in \Delta_{\mathbf{E}}: \widetilde{n \mathbf{u}}=\mathbf{v}\right\}$ do not intersect $\partial \Delta_{\mathbf{E}}$, and have equal $\lambda_{\Delta}$-measure by an obvious property of translation-invariance. There are $\left(\begin{array}{c}n-1 \\ |\mathbf{E}|-1\end{array}\right)$ elements in $n \Delta_{\mathbf{E}} \cap \mathbb{N}^{\mathbf{E}}$, which is the number of integer compositions of $n$ into $|\mathbf{E}|$ positive parts. We conclude that for any $\mathbf{v} \in n \Delta_{\mathbf{E}} \cap \mathbb{N}^{\mathbf{E}}$,

$$
0 \leq \lambda_{\Delta}\left(\Delta_{\mathbf{E}}\right)-\left(\begin{array}{c}
n-1 \\
|\mathbf{E}|-1
\end{array}\right) \lambda_{\Delta}\left(\left\{\mathbf{u} \in \Delta_{\mathbf{E}}: \widetilde{n \mathbf{u}}=\mathbf{v}\right\}\right) \leq \lambda_{\Delta}\left(V_{1 / n}\left(\partial \Delta_{\mathbf{E}}\right)\right),
$$

where $V_{1 / n}\left(\partial \Delta_{\mathbf{E}}\right)$ is the $1 / n$-neighborhood of $\partial \Delta_{\mathbf{E}}$, and $\lambda_{\Delta}\left(V_{1 / n}\left(\partial \Delta_{\mathbf{E}}\right)\right) \rightarrow 0$ as $n \rightarrow \infty$. Since

$$
\left(\begin{array}{c}
n-1 \\
|\mathbf{E}|-1
\end{array}\right) \sim n^{|\mathbf{E}|-1} /(|\mathbf{E}|-1)=n^{|\mathbf{E}|-1} \lambda_{\Delta}\left(\Delta_{\mathbf{E}}\right),
$$

we conclude that $\lambda_{\Delta}\left(\left\{\mathbf{u} \in \Delta_{\mathbf{E}}: \widetilde{n \mathbf{u}}=\mathbf{v}\right\}\right)=(1+o(1)) / n^{|\mathbf{E}|-1}$ as $n \rightarrow \infty$ independently of $\mathbf{v} \in n \Delta_{\mathbf{E}} \cap \mathbb{N}^{\mathbf{E}}$, and since $\left|n \partial \Delta_{\mathbf{E}} \cap \mathbb{Z}^{\mathbf{E}}\right|=o\left(n^{|\mathbf{E}|-1}\right)$, the conclusion follows easily from (15).

Lemma 8. - Set $\varsigma=(8 / 9)^{1 / 4}$. Let $G_{a}, a>0$ be a uniformly bounded family of functions on $\mathbb{N}^{\mathbf{E}} \times \mathbb{Z}^{E}$ such that

$$
G_{a}\left(\mathbf{r}_{a}, \mathbf{x}_{a}\right) \rightarrow G(\mathbf{r}, \mathbf{x})
$$

whenever $\mathbf{r}_{a} /(2 a)^{1 / 2} \rightarrow \mathbf{r}$ and $\mathbf{x}_{a} /\left(\varsigma a^{1 / 4}\right) \rightarrow \mathbf{x} \in \mathbb{R}^{E}$. Let also $\rho_{a} /(2 a)^{1 / 2} \rightarrow \rho$ with $\rho_{a} \in \mathbb{N}$.

Then

$$
\frac{\varsigma^{k+2 g-1}}{2^{\frac{|\mathbf{E}|-1}{2}} a^{\frac{|V|+|\mathbf{E}|-3}{4}}} \widehat{\mathbf{W N}}_{\mathfrak{m}}\left(G_{a} \mathbb{1}_{\left\{\rho(\mathbf{r})=\rho_{a}\right\}}\right) \rightarrow \widehat{\mathrm{PN}}_{\mathfrak{m}}^{(\rho)}(G)
$$

Proof. - Let $\mathbf{W}_{0 \rightarrow x}^{r}$ be the set of walks with duration $r$ that end at $x$, and $p_{n}(N)=3^{-r}\left|\mathbf{W}_{0 \rightarrow N}^{r}\right|$ be the probability that a uniform walk of duration $r$ ends at $N$. Note that $k+2 g-1=|\mathbf{E}|-|V|+1$ by (6), and write the left-hand side in the statement as

$$
\begin{aligned}
& \frac{\varsigma^{|\mathbf{E}|-|V|+1}}{2^{\frac{|\mathbf{E}|-1}{2}} a^{\frac{|V|+|\mathbf{E}|-3}{4}}} \sum_{\mathbf{r} \in \mathbb{N}^{\mathbf{E}}} 3^{-\sum_{\mathbf{e} \in \mathbf{E}} r_{\mathbf{e}}} \sum_{\mathbf{y} \in \mathbb{Z}^{V} \backslash\left\{v_{*}\right\}} \sum_{\left(w^{\mathfrak{e}}\right) \in \mathbf{W N}_{\mathfrak{m}}(\nabla \mathbf{y})} G_{a}(\mathbf{r}, \nabla \mathbf{y}) \mathbb{1}_{\left\{\rho(\mathbf{r})=\rho_{a}\right\}} \\
& =\frac{\varsigma^{|\mathbf{E}|-|V|+1}}{2^{\frac{|\mathbf{E}|-1}{2}} a^{\frac{|V|+|\mathbf{E}|-3}{4}}} \sum_{\mathbf{r} \in \rho_{a} \Delta_{\mathbf{E} \cap \mathbb{N}^{\mathbf{E}}}} \sum_{\mathbf{y} \in \mathbb{Z}^{V \backslash\left\{v_{*}\right\}}} G_{a}(\mathbf{r}, \nabla \mathbf{y}) \prod_{\mathfrak{e} \in E_{1 / 2}} p_{r_{\mathfrak{e}}}(\nabla \mathbf{y}(\mathfrak{e})) \\
& \underset{a \rightarrow \infty}{\sim} \rho_{a}^{|\mathbf{E}|-1} \frac{\varsigma^{|\mathbf{E}|-|V|+1}}{2^{\frac{|\mathbf{E}|-1}{2}} a^{\frac{|V|+|\mathbf{E}|-3}{4}}} \int_{\Delta_{\mathbf{E}}} \lambda_{\Delta}(\mathrm{d} \mathbf{u}) \int_{\mathbb{R}^{V \backslash\left\{v_{*}\right\}}} \mathrm{d} \mathbf{y} G_{a}\left(\widetilde{\rho_{a} \mathbf{u}}, \nabla\lfloor\mathbf{y}\rfloor\right) \prod_{\mathfrak{e} \in E_{1 / 2}} p_{\widetilde{\rho_{a} \mathbf{u}(\mathfrak{e})}}(\nabla\lfloor\mathbf{y}\rfloor(\mathfrak{e})) \\
& \underset{a \rightarrow \infty}{\sim} \rho^{|\mathbf{E}|-1} \int_{\Delta_{\mathbf{E}}} \lambda_{\Delta}(\mathrm{d} \mathbf{u}) \int_{\mathbb{R}^{V \backslash\left\{v_{*}\right\}}} \mathrm{d} \mathbf{y} G_{a}\left(\widetilde{\rho_{a} \mathbf{u}}, \nabla\left\lfloor a^{1 / 4} \varsigma \mathbf{y}\right\rfloor\right) \prod_{\mathfrak{e} \in E_{1 / 2}} \varsigma a^{1 / 4} p_{\widetilde{\rho_{a} \mathbf{u}(\mathfrak{e})}}\left(\nabla\left\lfloor a^{1 / 4} \varsigma \mathbf{y}\right\rfloor(\mathfrak{e})\right),
\end{aligned}
$$


where we made use of Lemma 7 for $n=\rho_{a}$ at the third step of the computation. Here, $\lfloor\mathbf{x}\rfloor$ is the vector whose components are the integer parts of the components of $\mathbf{x}$, and we write $\widetilde{\mathbf{u}}(\mathfrak{e})$ for the coordinate of $\widetilde{\mathbf{u}}$ corresponding to the edge $\mathbf{e}=\{\mathfrak{e}, \overline{\mathfrak{e}}\}$.

By the local limit theorem [39, Theorem VII.3.16], there exists ${ }^{(5)}$ some $C \in(0, \infty)$ such that for every $n \geq 1, N \in \mathbb{Z}$ and letting $x=N /\left(n^{1 / 2} \sqrt{2 / 3}\right)$,

$$
\left|\sqrt{\frac{2}{3}} n^{1 / 2} p_{n}(N)-\frac{1}{\sqrt{2 \pi}} e^{-\frac{x^{2}}{2}}\right| \leq n^{-1 / 2} \frac{C}{1+|x|^{2}} .
$$

From this, and the easily checked fact that $n \partial \Delta_{\mathbf{E}}$ is at distance $C_{\mathbf{E}}>0$ (independent on $n$ ) of the set $\left\{n \mathbf{u}: \mathbf{u} \in \Delta_{\mathbf{E}}: \widetilde{n \mathbf{u}} \notin n \partial \Delta_{\mathbf{E}}\right\}$ (so that the components $n u_{\mathfrak{e}}$ are bounded away from 0 for $n \mathbf{u}$ in this set), it follows that

$$
\varsigma a^{1 / 4} p_{\widetilde{\rho_{a} \mathbf{u}(\mathfrak{e})}}\left(\nabla\left\lfloor a^{1 / 4} \varsigma \mathbf{y}\right\rfloor(\mathfrak{e})\right) \leq C \min \left(\frac{\sqrt{\rho}}{x_{\mathfrak{e}}^{2}}, \frac{1}{\sqrt{\rho u_{\mathfrak{e}}}}\right)
$$

where $x_{\mathfrak{e}}=|\nabla \mathbf{y}(\mathfrak{e})|$. Combining with the fact that the $G_{a}$ are uniformly bounded allows us to bound the last displayed double integral by

$$
C \max \left(\rho^{3|\mathbf{E}| / 2-1}, \rho^{|\mathbf{E}| / 2-1}\right) \int_{\nabla_{V}} \lambda_{\nabla}(\mathrm{d} \mathbf{x}) \int_{\Delta_{\mathbf{E}}} \lambda_{\Delta}(\mathrm{d} \mathbf{u}) \prod_{\mathfrak{e} \in E_{1 / 2}}\left(\frac{1}{x_{\mathfrak{e}}^{2}} \mathbb{1}_{\left\{x_{\mathfrak{e}}>1\right\}}+\frac{1}{\sqrt{u_{\mathfrak{e}}}} \mathbb{1}_{\left\{x_{\mathfrak{e}} \leq 1\right\}}\right) .
$$

Developing the product and integrating in $\mathbf{u}$, we finally obtain the bound

$$
C \max \left(\rho^{3|\mathbf{E}| / 2-1}, \rho^{|\mathbf{E}| / 2-1}\right) \int_{\nabla_{V}} \lambda_{\nabla}(\mathrm{d} \mathbf{x}) \prod_{\mathfrak{e} \in E_{1 / 2}} \min \left(x_{\mathfrak{e}}^{-2}, 1\right) .
$$

Taking a spanning tree $\mathfrak{a} \in \mathrm{ST}(\mathfrak{m})$ and using Lemma 6 , this is bounded by

$$
\max \left(\rho^{3|\mathbf{E}| / 2-1}, \rho^{|\mathbf{E}| / 2-1}\right) \int_{\mathbb{R}^{E_{1 / 2}(\mathfrak{a})}} \mathrm{d} \mathbf{x}\left(\sum_{\mathfrak{e} \in E_{1 / 2}(\mathfrak{a})} \min \left(x_{\mathfrak{e}}^{-2}, 1\right)\right)^{|\mathbf{E}|},
$$

and this is finite. Therefore, we can apply dominated convergence in the integral expression above and pass to the limit as $a \rightarrow \infty$ to get

$$
\begin{aligned}
\frac{\varsigma^{|\mathbf{E}|-|V|+1}}{2^{\frac{|\mathbf{E}|-1}{2}} a^{\frac{|V|+|\mathbf{E}|-3}{4}}} & \widehat{W N}_{\mathfrak{m}}\left(G_{a} \mathbb{1}_{\rho(\mathbf{r})=\rho_{a}}\right) \\
& \underset{a \rightarrow \infty}{\longrightarrow} \rho^{|\mathbf{E}|-1} \int_{\Delta_{\mathbf{E}}} \lambda_{\Delta}(\mathrm{d} \mathbf{u}) \int_{\nabla_{V}} \lambda_{\nabla}(\mathrm{d} \mathbf{x}) G(\rho \mathbf{u}, \mathbf{x}) \prod_{\mathfrak{e} \in E_{1 / 2}} \frac{e^{-x_{\mathfrak{e}}^{2} /\left(2 \rho u_{\mathfrak{e}}\right)}}{\sqrt{2 \pi \rho u_{\mathfrak{e}}}} \\
& =\rho^{|\mathbf{E}|-1} \int_{\Delta_{\mathbf{E}}} \lambda_{\Delta}(\mathrm{d} \mathbf{u}) \int_{\nabla_{V}} \lambda_{\nabla}(\mathrm{d} \mathbf{x}) \frac{\exp \left(-\frac{\mathcal{E}_{\rho \mathbf{u}}(\mathbf{x})}{2}\right)}{\prod_{\mathbf{e} \in \mathbf{E}} \sqrt{2 \pi \rho u_{\mathbf{e}}}} G(\rho \mathbf{u}, \mathbf{x}),
\end{aligned}
$$

and the result follows from the definition of $\mathcal{N}_{\mathbf{r}}^{\mathfrak{m}}$ and $\mathcal{Z}^{\mathfrak{m}}(\mathbf{r})$ and from Lemma 5.

Lemma 9. - Let $G_{a}, a>0$ be functions $\mathbb{N}^{\mathbf{E}} \times \mathbb{Z}^{E} \rightarrow \mathbb{R}$ such that

$$
\left|G_{a}(\mathbf{r}, \mathbf{x})\right| \leq K \exp \left(-c a^{-1 / 2} \sum_{\mathbf{e} \in \mathbf{E}} r_{\mathbf{e}}\right)
$$

(5) Until the end of the proof, the same notation $C$ will be used for different such bounding constants. 
for some $K, c>0$, and satisfying the same convergence assumptions as in the previous lemma. Then

$$
\frac{\varsigma^{k+2 g-1}}{2^{\frac{|\mathbf{E}|}{2}} a^{\frac{|V|+|\mathbf{E}|-1}{4}}} \widehat{\mathrm{WN}}_{\mathfrak{m}}\left(G_{a}\right) \underset{a \rightarrow \infty}{\longrightarrow} \widehat{\mathrm{PN}}_{\mathfrak{m}}(G)
$$

Proof. - This is an immediate consequence of the previous lemma, using easy dominated convergence arguments along with the $\rho$-dependent bound (16). Details are left to the reader.

4.1.3. Path network measure. - We are now able to describe the scaling limit of the measure $\mathrm{WN}_{\mathfrak{m}}$. Let $P_{0 \rightarrow x}^{r}(\mathrm{~d} w)$ be the law of the standard Brownian bridge with duration $t$ from 0 to $x$, and $P_{0 \rightarrow x}^{r}\left(\mathrm{~d} w \mathrm{~d} w^{\prime}\right)$ be the image measure of $P_{0 \rightarrow x}^{r}(\mathrm{~d} w)$ under $w \mapsto(w, \bar{w}) \in \mathcal{W}^{2}$.

Take an orientation $E_{1 / 2}$ of $E$. We define a measure by

$$
\operatorname{PN}_{\mathfrak{m}}\left(\mathrm{d}\left(w^{\mathfrak{e}}, \mathfrak{e} \in E\right)\right)=\int \widehat{\mathrm{PN}}_{\mathfrak{m}}(\mathrm{d} \mathbf{r}, \mathrm{d} \mathbf{x}) \prod_{\mathfrak{e} \in E_{1 / 2}} P_{0 \rightarrow x_{e}}^{r_{e}}\left(\mathrm{~d} w^{\mathfrak{e}} \mathrm{d} w^{\overline{\mathfrak{e}}}\right),
$$

which is a measure on paths networks, i.e. collections $\left(w^{\mathfrak{e}}, \mathfrak{e} \in E\right)$ of paths with durations $r_{\mathfrak{e}}:=\tau\left(w^{\mathfrak{e}}\right)$ such that $w^{\overline{\mathfrak{e}}}=\bar{w}^{\mathfrak{e}}$ and $\left(\widehat{w}_{\mathfrak{e}}, \mathfrak{e} \in E\right) \in \nabla_{V}$ is a potential. This measure does not depend on the choice of $E_{1 / 2}$ because of the well-known invariance property [41] of $P_{0 \rightarrow x}^{t}$ under $w \mapsto \bar{w}$.

We define a scaling operator $\phi^{a}$ on $\mathcal{W}$ by

$$
\phi^{a}(w)=\left(\frac{9}{8 a}\right)^{1 / 4} w\left((2 a)^{1 / 2} s\right), \quad 0 \leq s \leq \tau(w) /(2 a)^{1 / 2} .
$$

We extend the scaling function $\phi^{a}$ to a function on powers of $\mathcal{W}$ in the natural way, i.e. it acts on every component by applying the scaling $\phi^{a}$.

Proposition 2. - Let $H$ be a continuous function $\mathcal{W}^{E} \rightarrow \mathbb{R}$ such that

$$
\left|H\left(\left(w^{\mathfrak{e}}, \mathfrak{e} \in E\right)\right)\right| \leq K \exp \left(-c \sum_{\mathfrak{e} \in E} \tau\left(w^{\mathfrak{e}}\right)\right),
$$

for some $K, c>0$. Then

$$
\frac{\varsigma^{k+2 g-1}}{2^{\frac{|\mathbf{E}|}{2}} a^{\frac{|V|+|\mathbf{E}|-1}{4}}} \mathrm{WN}_{\mathfrak{m}}\left(H \circ \phi^{a}\right) \underset{a \rightarrow \infty}{\longrightarrow} \mathrm{PN}_{\mathfrak{m}}(H) .
$$

Proof. - We rely on Lemma 9 and the following statement.

Lemma 10. - For $n \in \mathbb{N}$ and $x \in \mathbb{Z}$, let $\mathbf{P}_{0 \rightarrow x}^{n}$ be the uniform distribution on $\mathbf{W}_{0 \rightarrow x}^{n}$. Let $r>0, x \in \mathbb{R}$ be fixed and assume that $r_{a}, x_{a}$ are integers such that $r_{a} /(2 a)^{1 / 2} \rightarrow r$ and $(9 / 8 a)^{1 / 4} x_{a} \rightarrow x$. Then the probability measures $\phi_{*}^{a} \mathbf{P}_{0 \rightarrow x_{a}}^{r_{a}}$ converge in distribution to $P_{0 \rightarrow x}^{r}$ in $\mathcal{W}$.

Proof. - This is a classical elaboration on Donsker's invariance principle. See for instance [13, Lemmas 4.5-6] for the scheme of the proof (in this reference, the author considers more general stable processes than Brownian motion but is also concerned only in the case where $x=0$, however the proof in the general $x$ case is the same). 
To end the proof of Proposition 2, we apply Lemma 9 to the function

$$
G_{a}(\mathbf{r}, \mathbf{x})=\int_{\mathcal{W}^{E}} \bigotimes_{\mathfrak{e} \in E_{1 / 2}} \mathbf{P}_{0 \rightarrow x_{\mathfrak{e}}}^{r_{\mathfrak{e}}}\left(\mathrm{d} w^{\mathfrak{e}} \mathrm{d} w^{\overline{\mathfrak{e}}}\right) H\left(\phi^{a}\left(w^{\mathfrak{e}}, \mathfrak{e} \in E\right)\right),
$$

where as above the measure $\mathbf{P}_{0 \rightarrow x}^{r}\left(\mathrm{~d} w \mathrm{~d} w^{\prime}\right)$ is the law of $(w, \bar{w})$ under $\mathbf{P}_{0 \rightarrow x}^{r}$.

Remark. - The path network measure $\mathrm{PN}_{\mathfrak{m}}$ actually has the following interpretation, which we state without proof as this is not needed in the present article. Assume that the numbers $r_{\mathbf{e}}>0, \mathbf{e} \in \mathbf{E}$ are given and consider a graph with same structure as $\mathfrak{m}$ but where the edge $\mathbf{e}$ is isometric to a real segment of length $r_{\mathbf{e}}$. This defines a metric graph [9, Chapter 3.2.2] $\mathfrak{m}_{\mathbf{r}}$. Let $\Theta_{\mathfrak{m}_{\mathbf{r}}}$ be the set of continuous functions on $\mathfrak{m}_{\mathbf{r}}$ that vanish at $v_{*}$. With any path network $\left(w^{\mathfrak{e}}, \mathfrak{e} \in E\right)$ we may associate an element of $\Theta_{\mathfrak{m}_{\mathbf{r}}}$ in the following way: for $x \in \mathfrak{m}_{\mathbf{r}}$ on the half-edge $e$ and at distance $0 \leq s \leq r_{\mathbf{e}}$ from its origin, pick a chain $e_{1}, \ldots, e_{m}$ of half edges starting at $v_{*}$ and ending at $e^{-}$. Then let $H(x)=\sum_{i=1}^{m} \widehat{w}^{e_{i}}+w^{\mathfrak{e}}(s)$. The reader will convince himself that this definition does not depend on the choice of the orientation of $e$ nor on that of the chain $e_{1}, \ldots, e_{m}$, because of the properties of path networks. Thus, the measure

$$
\mathcal{N}_{\mathbf{r}}^{\mathfrak{m}}(\mathrm{d} \mathbf{x}) \prod_{\mathfrak{e} \in E_{1 / 2}} P_{0 \rightarrow x_{\mathfrak{e}}}^{r_{\mathfrak{e}}}\left(\mathrm{d} w^{\mathfrak{e}} \mathrm{d} w^{\bar{e}}\right)
$$

induces a law $\operatorname{GFF}_{\mathfrak{m}_{\mathbf{r}}}(\mathrm{d} H)$ on $\Theta_{\mathfrak{m}_{\mathbf{r}}}$. This law is what is referred to as the Gaussian free field on $\mathfrak{m}_{\mathbf{r}}$. We refer to [43] for a nice introduction to this object (here the metric graphs are really 1-dimensional objects, so the associated free fields are well-defined random functions that are much less elaborate than higher-dimensional free fields on which [43] focuses). To explain what GFF is, imagine that we run a "Brownian motion" on $\mathfrak{m}_{\mathbf{r}}$. It behaves as a linear Brownian motion when moving on an edge of $\mathfrak{m}_{\mathbf{r}}$, and when it encounters a vertex of the graph, its excursions out of the vertex choose independently at random in which incident edge they will diffuse. The diffusion is killed when it first encounters the vertex $v_{*}$. We let $G(x, y)$ be the density ${ }^{(6)}$ at vertex $y$ of the occupation measure of the diffusion starting at $x$. Then $\mathrm{GFF}_{\mathfrak{m}_{\mathbf{r}}}$ is the law of the centered Gaussian field $\left(H(x), x \in \mathfrak{m}_{\mathbf{r}}\right)$ with covariance

$$
\operatorname{Cov}(H(x), H(y))=G(x, y), \quad x, y \in \mathfrak{m}_{\mathbf{r}} .
$$

\subsection{Discrete and continuous snakes}

We now discuss the scaling limits of the measures $\mathrm{DS}_{r}$ on discrete snakes. This is more classical from a probabilistic point of view as we are back into the realm of probability measures. We define the continuous snake measures $\mathrm{S}_{r}, r>0$, as the law of the pair $\left(\left(B_{s}\right)_{0 \leq s \leq I_{r}^{-1}},\left(S_{s}\right)_{0 \leq s \leq I_{r}^{-1}}\right)$, where

- $\left(B_{s}, s \geq 0\right)$ is a standard Brownian motion in $\mathbb{R}$,

- $\left(I_{s}=\inf _{0 \leq s^{\prime} \leq s} B_{s^{\prime}}, s \geq 0\right)$ is the infimum process of $B$ and $I^{-1}$ is its right-continuous inverse,

- conditionally on $B$, the process $S$ is centered Gaussian in $\mathbb{R}$, with covariance

$$
\operatorname{Cov}\left(S_{s}, S_{s^{\prime}}\right)=\inf _{s \wedge s^{\prime} \leq s^{\prime \prime} \leq s \vee s^{\prime}}\left(B_{s^{\prime \prime}}-I_{s^{\prime \prime}}\right) .
$$

\footnotetext{
$\overline{\text { (6) The function } y} \mapsto G(x, y)$ can also be defined as the solution of a Poisson equation $\Delta_{\mathfrak{m}_{\mathbf{r}}} H=\delta_{x}$ vanishing at $v_{*}$, for the appropriate definition of the Laplacian $\Delta_{\mathfrak{m}_{\mathbf{r}}}$ on regular functions of $\Theta_{\mathfrak{m}_{\mathbf{r}}}$. 
As an application of Kolmogorov's continuity criterion and the standard fact that trajectories of a Brownian motion are a.s. locally Hölder continuous, a continuous version of $S$ exists, and $\mathrm{S}_{t}$ is a distribution on $\mathcal{W}^{2}$.

We rely on a version of invariance principles such as [11,23, 31], but holding for forests. It is easily derived from Theorem 3 of [35] by using the methods of [14, Section 2.4] to derive the results for the contour process of the tree rather than the height process. There is a difference between the label processes used in this reference and the present paper (due to a different way of exploring the trees), but the proofs are adapted in a straightforward way.

We define the scaling functions $\vartheta^{a}, \psi^{a}$ on $\mathcal{W}$ by

$$
\vartheta^{a}(c)=\frac{1}{(2 a)^{1 / 2}} c(2 a s), \quad 0 \leq s \leq \tau(c) / 2 a,
$$

and

$$
\psi^{a}(z)=\left(\frac{9}{8 a}\right)^{1 / 4} z(2 a s), \quad 0 \leq s \leq \tau(z) / 2 a .
$$

Lemma 11. - Fix $t>0$ and assume $r_{a} \in \mathbb{N}$ is such that $r_{a} /(2 a)^{1 / 2} \rightarrow r$. Then, the measure $\left(\vartheta^{a}, \psi^{a}\right)_{*} \mathrm{DS}_{r_{a}}$ converges weakly to $\mathrm{S}_{r}$ as $a \rightarrow \infty$. If $U_{n}$ is the uniform distribution over $\{0,1, \ldots, n-1\}$, we also have that the measure defined by

$$
\int \mathrm{DS}_{r_{a}}(\mathrm{~d}(c, z)) U_{\tau(c)}(\mathrm{d} t) H\left(\vartheta^{a}(c), \psi^{a}(z), t / 2 a\right),
$$

for positive measurable $H$, converges weakly towards the measure defined by

$$
\int \mathrm{S}_{r}(\mathrm{~d}(c, z)) \int_{\mathbb{R}_{+}} \frac{\mathrm{d} t \mathbb{1}_{\{0 \leq t \leq \tau(c)\}}}{\tau(c)} H(c, z, t) .
$$

\subsection{Scaling limits}

In this section, we assume $(g, k) \notin\{(0,1),(0,2)\}$. We define a measure on $\mathbf{C}_{g, k}$ by

$$
\mathrm{CLM}_{g, k}=2 \sum_{\mathfrak{m} \in \mathrm{M}_{g, k}^{3}} \mathrm{CLM}_{\mathfrak{m}}
$$

and $\mathrm{CLM}_{\mathfrak{m}}$ is the appropriate image measure obtained by rearranging terms of

$$
\delta_{\mathfrak{m}} \mathrm{PN}_{\mathfrak{m}}\left(\mathrm{d}\left(w^{\mathfrak{e}}\right)_{\mathfrak{e} \in E(\mathfrak{m})}\right) \bigotimes_{\mathfrak{e} \in E(\mathfrak{m})} \mathrm{S}_{r_{\mathfrak{e}}}\left(\mathrm{d}\left(c^{\mathfrak{e}}, z^{\mathfrak{e}}\right)\right) \mathrm{d} t^{*} \mathbb{1}_{\left\{0 \leq t^{*} \leq \tau^{\mathfrak{e} *}\right\}}
$$

Note that the exponent $\geq 3$ of the first term defining $\mathrm{LM}_{g, k}$ is replaced by a 3 here. We define the scaling operator $\xi^{a}$ by

$$
\xi^{a}\left(\mathfrak{m}, \mathfrak{l}, t^{*}\right)=\left(\mathfrak{m},\left(\left(\phi^{a}\left(w^{\mathfrak{e}}\right), \vartheta^{a}\left(c^{\mathfrak{e}}\right), \psi^{a}\left(z^{\mathfrak{e}}\right)\right), \mathfrak{e} \in E(\mathfrak{m})\right), t^{*} / 2 a\right) .
$$

Theorem 5. - Assume $(g, k) \notin\{(0,1),(0,2)\}$. Let $H$ be a continuous function on $\mathbf{C}_{g, k}$ such that

$$
|H| \leq K \tau_{\mathfrak{l}}^{-1} \exp \left(-\beta \tau_{\mathfrak{l}}\right)
$$

for some $K, \beta>0$. Then it holds that

$$
\frac{\varsigma^{k+2 g-1}}{2^{3 g-2+\frac{3 k}{2}} a^{\frac{5 g}{2}+\frac{5 k-7}{4}}} \operatorname{LM}_{g, k}\left(H \circ \xi^{a}\right) \underset{a \rightarrow \infty}{\longrightarrow} \operatorname{CLM}_{g, k}(H) \text {. }
$$


Proof. - First of all, we check that for every $\mathbf{r} \in \mathbb{N}^{\mathbf{E}}$,

$$
\bigotimes_{\mathfrak{e} \in E} \mathrm{DS}_{r_{\mathfrak{e}}}\left(\exp \left(-\frac{\beta}{a} \tau_{\mathfrak{l}}\right)\right) \leq K^{\prime} \exp \left(-c_{\beta} a^{-1 / 2} \sum_{\mathbf{e} \in \mathbf{E}} r_{\mathbf{e}}\right) \text {. }
$$

Indeed, under $\mathrm{DS}_{r}(\mathrm{~d}(c, z))$, the terminal time $\tau$ of $c$ has same law as the first hitting time of $-r$ by a simple random walk on the integers. Thus $\tau_{\mathfrak{l}}$ has same law under $\bigotimes_{e \in E} \mathrm{DS}_{r_{e}}$ as the first hitting time of $-2 \sum_{\mathbf{e} \in \mathbf{E}} r_{\mathbf{e}}$ by the random walk. Standard computations show that

$$
\mathrm{DS}_{1}\left(s^{\tau(c)}\right)=\frac{1-\sqrt{1-s^{2}}}{s}, \quad 0<s \leq 1
$$

so that

$$
\bigotimes_{\mathfrak{e} \in E(\mathfrak{m})} \mathrm{DS}_{r_{\mathfrak{e}}}\left(\exp \left(-\frac{\beta}{a} \tau_{\mathfrak{l}}\right)\right)=\exp \left(-2 \phi\left(\frac{\beta}{a}\right) \sum_{\mathbf{e} \in \mathbf{E}} r_{\mathbf{e}}\right),
$$

where $\phi(\beta)=-\log \left(e^{\beta}\left(1-\sqrt{1-e^{-2 \beta}}\right)\right)$ is the Laplace exponent of the first hitting time of -1 by the walk, so $\phi(\beta / a) \sim(2 \beta / a)^{1 / 2}$ as $a \rightarrow \infty$, hence (18). We can thus write $\operatorname{LM}_{\mathfrak{m}}(H)$ as

$$
\int \mathrm{WN}_{\mathfrak{m}}\left(\mathrm{d}\left(w^{\mathfrak{e}}\right)\right) \int \bigotimes_{\mathfrak{e} \in E} \mathrm{DS}_{r_{\mathfrak{e}}}\left(\mathrm{d}\left(c^{\mathfrak{e}}, z^{\mathfrak{e}}\right)\right) \int U_{\tau^{\mathfrak{e} *}}\left(\mathrm{~d} t^{*}\right) \tau^{\mathfrak{e}_{*}} H\left(\mathfrak{m}, \mathfrak{l}, t^{*}\right)
$$

with the notation $U_{n}$ of Lemma 11. Set

$$
G_{a}(\mathbf{r}, \mathbf{x})=\bigotimes_{\mathfrak{e} \in E_{1 / 2}} \mathbf{P}_{0 \rightarrow x_{\mathfrak{e}}}^{r_{\mathfrak{e}}} \bigotimes_{\mathfrak{e} \in E} \operatorname{DS}_{r_{\mathfrak{e}}}\left[U_{\tau^{\mathfrak{e}_{*}}}\left(\frac{\tau^{\mathfrak{e}_{*}}}{2 a} H\left(\xi^{a}\left(\mathfrak{m}, \mathfrak{l}, t^{*}\right)\right)\right)\right]
$$

Because of the domination hypothesis on $H$, the fact $\tau^{\mathfrak{e}_{*}} \leq \tau_{\mathfrak{l}}$, and inequality (18), the function $G_{a}$ satisfies the domination hypothesis of Lemma 9. Applying the latter and Lemmas 10 and 11 allows us to conclude that for every $\mathfrak{m} \in \mathbf{M}_{g, k}^{\geq 3}$,

$$
(2 a)^{-1} \frac{\varsigma^{k+2 g-1}}{2^{\frac{|\mathbf{E}|}{2}} a^{\frac{|\mathbf{E}|+|V|-1}{4}}} \operatorname{LM}_{\mathfrak{m}}\left(H \circ \xi^{a}\right) \underset{a \rightarrow \infty}{\rightarrow} \operatorname{CLM}_{\mathfrak{m}}(H) .
$$

Note that for $\mathfrak{m}$ ranging in $\mathbf{M}_{g, k}^{\geq 3}$, the quantity $|V|+|\mathbf{E}|$ appearing in the scaling factor is maximal if and only if $\mathfrak{m}$ belongs to the set $\mathbf{M}_{g, k}^{3}$ of trivalent maps of genus $g$ with $k$ faces. Indeed, $|\mathbf{E}|-|V|=k+2 g-2$ is a fixed quantity, so that $|V|+|\mathbf{E}|$ will be maximal if and only if $|\mathbf{E}|$ is maximal subject to the constraints. On the other hand, we already noticed that $|\mathbf{E}| \leq 3 k+6 g-6$, with equality if and only if $\mathfrak{m} \in \mathbf{M}_{g, k}^{3}$, in which case $(|V|+|\mathbf{E}|+3) / 4=5 g / 2+(5 k-7) / 4$. Since $\mathbf{M}_{g, k}^{\geq 3}$ is a finite set, we deduce that the only terms in the sum (14) remaining in the scaling limit will be those belonging to $\mathbf{M}_{g, k}^{3}$. Hence the result.

Remark: the Hurwitz measure. It should be noted that a non-labeled analog of $\mathrm{CLM}_{g, k}$ has already been considered in the literature [37, 38], in problems dealing with the enumeration of maps, random matrices, and related algebraic geometry problems. Specifically, the first marginal $\mathrm{P}_{t}$ of the measure $\mathrm{S}_{t}$ can be considered as the law of the contour process of a random forest. The measure considered in the above references is

$$
\mathrm{CM}_{g, k}^{\circ}:=\sum_{\mathfrak{m} \in \mathbf{M}_{g, k}^{3, \circ}} \frac{1}{|\operatorname{Aut}(\mathfrak{m})|} \int_{\mathbb{R}_{+}^{\mathbf{E}}} \mathrm{d} \mathbf{r} \bigotimes_{\mathfrak{e} \in E} \mathrm{P}_{r_{\mathfrak{e}}}\left(\mathrm{d} c^{\mathfrak{e}}\right),
$$


where the first sum is over unrooted maps in $\mathbf{M}_{g, k}^{3}$ (but still with indexed faces) and the factor involving Aut $(\mathfrak{m})$, the automorphisms of $\mathfrak{m}$, allows one to get rid of symmetries of the graph. Note that we can regroup the factors in the above measure as $\bigotimes_{\mathfrak{e} \in E_{1 / 2}} \mathrm{~d} r_{\mathfrak{e}} \mathrm{P}_{r_{\mathfrak{e}}}\left(\mathrm{d} c^{\mathfrak{e}}\right) \mathrm{P}_{r_{\mathfrak{e}}}\left(\mathrm{d} c^{\overline{\mathfrak{e}}}\right)$. In this form, and by using an interpretation in terms of trees of a well-known decomposition of the Brownian excursion due to Bismut (see Le Gall [25]), we see that we have replaced each edge $\mathfrak{m}$ by a doubly-marked Brownian continuum random tree taken under the Itô measure, using the two marks as endpoints of the edge. The measure $\mathrm{CM}_{g, k}^{\circ}$ appears as a scaling limit of a random element of $\mathbf{M}_{g, k}$ where $k$ remains fixed but the number of edges tends to infinity. Of particular importance is the Hurwitz measure, which is the image of $\mathrm{CM}_{g, k}^{\circ}$ by the map

$$
\left(c^{\mathfrak{e}}, \mathfrak{e} \in E\right) \longmapsto\left(\sum_{e \in f_{i}(\mathbf{m})} \tau\left(c^{\mathfrak{e}}\right), 1 \leq i \leq k\right),
$$

as its Laplace transform has an interpretation in terms of generating series related to intersection theory on the moduli space of curves [38].

\subsection{Exceptional cases}

This section is devoted to the study of the planar cases $(g, k) \in\{(0,1),(0,2)\}$. An element $(\mathbf{m},[\mathbf{l}])$ of $\mathbf{L M}_{0,1}$ is just a rooted labeled tree and is described by a snake $\Xi_{0,1}(\mathbf{m},[\mathbf{l}])=(c, z) \in \mathbf{S}_{1}$. The image measure of $Q_{0,1}$ by $\widetilde{\Psi}_{0,1}:=\Xi_{0,1} \circ \Psi_{0,1}$ will assign mass $2 \cdot 12^{-n}=4 \cdot 2^{-2 n-1} 3^{-n}$ to each discrete snake $(c, z)$ with duration $\tau(c)=2 n+1$, coding a labeled tree with $n$ edges. This is precisely the measure

$$
\mathrm{LM}_{0,1}:=4 \mathrm{DS}_{1} \text {. }
$$

We define its scaling limit $\operatorname{CLM}_{0,1}$ as follows. Let $N(\mathrm{~d} c)$ be the Itô measure on $\mathcal{W}$, governing the intensity of the excursions of standard reflected Brownian motion (with normalization $\left.N(\tau(c) \in \mathrm{d} t)=\left(2 \pi t^{3}\right)^{-1 / 2} \mathrm{~d} t\right)$. Let $\mathbf{N}(\mathrm{d}(c, z))$ be the measure on $\mathcal{W}^{2}$ such that under $\mathbf{N}$, $c$ has "law" $N$, and conditionally on $c, z$ is a centered Gaussian process with covariance

$$
\operatorname{Cov}\left(z(s), z\left(s^{\prime}\right)\right)=\inf _{s \wedge s^{\prime} \leq s^{\prime \prime} \leq s \vee s^{\prime}} c\left(s^{\prime \prime}\right) .
$$

Finally, set $\mathrm{CLM}_{0,1}=4 \mathbf{N}$.

Proposition 3. - Let $H$ be a continuous functional on $\mathcal{W}^{2}$ such that

$$
|H(c, z)| \leq K \tau \exp (-\beta \tau),
$$

where $\tau:=\tau(c)$, for some $K, \beta>0$. Then

$$
(2 a)^{1 / 2} \operatorname{LM}_{0,1}\left(H \circ\left(\vartheta^{a}, \psi^{a}\right)\right) \underset{a \rightarrow \infty}{\longrightarrow} \operatorname{CLM}_{0,1}(H) .
$$

Proof. - First integrate out the label part, i.e. assume $H$ is only a function of the first component and write

$$
\begin{aligned}
(2 a)^{1 / 2} \operatorname{LM}_{0,1}\left(H\left(\vartheta^{a}(c)\right)\right) & =2(2 a)^{1 / 2} \int_{\mathbb{R}_{+}} \mathrm{d} t \frac{1}{2^{2\lceil t\rceil}} \operatorname{Cat}_{\lceil t\rceil} P_{\operatorname{exc}}^{\lceil t\rceil}\left(H\left(\vartheta^{a}(c)\right)\right) \\
& =2 \sqrt{2} a^{3 / 2} \int_{\mathbb{R}_{+}} \mathrm{d} t \frac{1}{2^{2\lceil a t\rceil}} \operatorname{Cat}_{\lceil a t\rceil} P_{\operatorname{exc}}^{\lceil a t\rceil}\left(H\left(\vartheta^{a}(c)\right)\right)
\end{aligned}
$$


where $P_{\mathrm{exc}}^{n}$ is the uniform distribution over Dyck paths with duration $2 n+1$, the latter set having cardinality $\mathrm{Cat}_{n}$, the $n$-th Catalan number. It is well-known (and can be obtained from Lemma 10 with $x=-1$ from a cyclic shift and Vervaat's theorem [40]) that the law of $\vartheta^{a}(c)$ under $P_{\text {exc }}^{\lceil a t\rceil}$ converges weakly to $N^{(t)}=N(\cdot \mid \tau=t)$. On the other hand, it can be checked that $4^{-n}(n+1) \sqrt{n}$ Cat $_{n}$ increases in $n$ towards its limit $\pi^{-1 / 2}$, so that $a^{3 / 2} 2^{-2\lceil a t\rceil} \mathrm{Cat}_{\lceil a t\rceil} P_{\operatorname{exc}}^{\lceil a t\rceil}\left(H\left(\vartheta^{a}(c)\right)\right)$ is dominated by $K^{\prime} e^{-2 \beta t} / \sqrt{t}$ for some $K^{\prime}>0$. By dominated convergence, we get

$$
(2 a)^{1 / 2} \mathrm{LM}_{0,1}\left(H \circ \vartheta^{a}\right) \underset{a \rightarrow \infty}{\longrightarrow} 4 \int_{\mathbb{R}_{+}} \frac{\mathrm{d} t}{\sqrt{2 \pi t^{3}}} N^{(t)}(H)=4 N(H)
$$

by the fact that $N(\tau \in \mathrm{d} t)=\mathrm{d} t / \sqrt{2 \pi t^{3}}$. Incorporating the spatial displacements is now easy from the convergence of the contour function, see for instance [23].

Finally, the measure associated with $Q_{0,2}$ through the mapping $\widetilde{\Psi}_{0,2}$ is the measure $\mathrm{LM}_{0,2}=2 \sum_{\mathfrak{m} \in \mathbf{M}_{0,2}^{\geq 3}} \mathrm{LM}_{\mathfrak{m}}$ where

$$
\operatorname{LM}_{\mathfrak{m}}=\delta_{\mathfrak{m}} \mathrm{WN}_{\mathfrak{m}}\left(\mathrm{d} w^{\mathfrak{e}_{*}} \mathrm{~d} w^{\overline{\mathfrak{e}}_{*}}\right) \mathrm{DS}_{1}(\mathrm{~d}(c, z)) \#_{\tau}\left(\mathrm{d} t^{*}\right) \operatorname{DS}_{r_{\mathfrak{e}} *-1}\left(\mathrm{~d}\left(c^{\mathfrak{e}_{*}}, z^{\mathfrak{e}_{*}}\right)\right) \operatorname{DS}_{r_{\mathfrak{e}} *}\left(\mathrm{~d}\left(c^{\overline{\mathfrak{e}}_{*}}, z^{\overline{\mathfrak{e}}_{*}}\right)\right) .
$$

Its continuous counterpart is of course $\mathrm{CLM}_{0,2}=2 \sum_{\mathfrak{m} \in \mathrm{M}_{0,2}^{\geq 3}} \mathrm{CLM}_{\mathfrak{m}}$, where

$$
\mathrm{CLM}_{\mathfrak{m}}=\delta_{\mathfrak{m}} \mathrm{PN}_{\mathfrak{m}}\left(\mathrm{d} w^{\mathfrak{e}_{*}} \mathrm{~d} w^{\overline{\mathfrak{e}}_{*}}\right) \mathbf{N}(\mathrm{d}(c, z)) \mathrm{d} t^{*} \mathbb{1}_{\left\{0 \leq t^{*} \leq \tau\right\}} \mathrm{S}_{r_{\mathfrak{e}_{*}}}\left(\mathrm{~d}\left(c^{\mathfrak{e}_{*}}, z^{\mathfrak{e}_{*}}\right)\right) \mathrm{S}_{r_{\mathfrak{e}^{*}}}\left(\mathrm{~d}\left(c^{\overline{\mathfrak{e}}_{*}}, z^{\overline{\mathfrak{e}}_{*}}\right)\right),
$$

and $\mathrm{PN}_{\mathfrak{m}}$ takes the particularly simple form

$$
\mathrm{PN}_{\mathfrak{m}}\left(\mathrm{d} w^{\mathfrak{e}_{*}} \mathrm{~d} w^{\overline{\mathfrak{e}}_{*}}\right)=\int_{\mathbb{R}_{+}} \frac{\mathrm{d} r}{\sqrt{2 \pi r}} P_{0 \rightarrow 0}^{r}\left(\mathrm{~d} w^{\mathfrak{e}_{*}} \mathrm{~d} w^{\overline{\mathfrak{e}}_{*}}\right) .
$$

We define the scaling operation $\xi^{a}$ by

$\xi^{a}\left(\mathfrak{m}, \mathfrak{l}, t^{*}\right)=\left(\mathfrak{m},\left(\vartheta^{a}(c), \psi^{a}(z)\right),\left(\phi^{a}\left(w^{\mathfrak{e}_{*}}\right), \vartheta^{a}\left(c^{\mathfrak{e}_{*}}\right), \psi^{a}\left(z^{\mathfrak{e}_{*}}\right)\right),\left(\phi^{a}\left(w^{\overline{\mathfrak{e}}_{*}}\right), \vartheta^{a}\left(c^{\overline{\mathfrak{e}}_{*}}\right), \psi^{a}\left(z^{\overline{\mathfrak{e}}_{*}}\right)\right), t^{*} / 2 a\right)$.

Proposition 4. - For every continuous functional H satisfying

$$
|H| \leq K \exp \left(-\beta \tau_{\mathfrak{l}}\right)
$$

for some $K, \beta>0$. Then

$$
\frac{\varsigma}{2 a^{3 / 4}} \operatorname{LM}_{0,2}\left(H \circ \xi^{a}\right) \underset{a \rightarrow \infty}{\longrightarrow} \operatorname{CLM}_{0,2}(H)
$$

The proof uses both the ingredients of the proofs of Theorem 5 and Proposition 3, but presents no new difficulty and is left as an exercise to the reader.

\section{Asymptotic enumeration in the non-planar case}

We want to derive Theorem 1 by a study of the generating function

$$
G(s):=\sum_{n \geq 1} \frac{\left|\mathbf{Q}_{g}^{n}\right|}{12^{n}} s^{n},
$$


which will be done by elementary singularity analysis. We will restrict our attention to the non-planar cases $g \geq 1$, as the planar case is already known. First note that we have the following alternative expressions

$$
G(s)=\sum_{\mathbf{q} \in \mathbf{Q}_{g}}\left(\frac{s}{12}\right)^{|F(\mathbf{q})|}=\sum_{(\mathbf{q}, x) \in \mathbf{Q}_{g, 1}} V_{\mathbf{q}}^{-1}\left(\frac{s}{12}\right)^{|F(\mathbf{q})|}=\mathbf{Q}_{g, 1}\left(V_{\mathbf{q}}^{-1} s^{|F(\mathbf{q})|}\right),
$$

which is finally equal to $\operatorname{LM}_{g, 1}\left(\left(\tau_{\mathfrak{l}} / 2+\chi(g)\right)^{-1} s^{\tau_{\mathfrak{l}} / 2}\right)$. The coefficients' asymptotic behavior is the same as that of $G_{0}(s):=\operatorname{LM}_{g, 1}\left(2 \tau_{\mathfrak{l}}^{-1} s^{\tau_{\mathfrak{l}} / 2}\right)$, so we may and will work with the latter. Now write $s=e^{-1 / a}, H=\tau_{\mathfrak{l}}^{-1} e^{-\tau_{\mathfrak{l}}}$, and apply Theorem 5 . We readily obtain that

$$
G_{0}\left(e^{-1 / a}\right) \underset{a \rightarrow \infty}{\sim} C_{g}^{\prime} a^{\frac{5 g-3}{2}},
$$

where $C_{g}^{\prime} \in(0, \infty)$ is defined by $C_{g}^{\prime}=2^{\frac{3 g-1}{2}} 3^{g} \operatorname{CLM}_{g, 1}\left(\tau_{\mathfrak{l}}^{-1} e^{-\tau_{\mathfrak{l}}}\right)$. Otherwise said,

$$
G_{0}(s) \sim C_{g}^{\prime}(1-s)^{-(5 g-3) / 2}
$$

as $s \rightarrow 1$ along $[0,1]$. From this, Tauberian theorems allow to obtain

$$
\sum_{k=1}^{n} 12^{-n}\left|\mathbf{Q}_{g}^{n}\right| \sim \frac{C_{g}^{\prime}}{\Gamma\left(\frac{5 g-1}{2}\right)} n^{\frac{5 g-3}{2}},
$$

which is weaker than (but consistent with) what we are aiming at. In order to apply standard transfer theorems of singularity analysis, we need to show that the asymptotics (20) hold for $s \rightarrow 1$ in a domain of the form

$$
D_{R, \phi}:=\{s \in \mathbb{C}:|s|<R, s \neq 1,|\arg (s-1)|>\phi\}
$$

for some $R>1, \phi \in(0, \pi / 2)$ and on which $G_{0}$ has to be extendible analytically (here we take the determination of the argument in $(-\pi, \pi])$.

Lemma 12. - Let $\alpha>0$ and $f(z)=\sum_{n \geq 0} h_{n} z^{n}$ be a power series with non-negative coefficients satisfying $h_{n} \sim n^{\alpha-1} / \Gamma(\alpha)$ as $n \rightarrow \infty$. Then $f(z) \sim(1-z)^{-\alpha}$ for $z \rightarrow 1$ inside a domain of the form $D_{1, \phi}$ with $\phi \in(\pi / 2, \pi)$.

Proof. - Let $(1-z)^{-\alpha}=\sum_{n \geq 0} h_{n}^{0} z^{n}$, so that $h_{n}^{0} \sim h_{n}$. Fix $\varepsilon>0$, take $N$ large enough so that $\left|1-h_{n} / h_{n}^{0}\right| \leq \varepsilon$. From the fact that $h_{n}, h_{n}^{0} \geq 0$, we easily get

$$
\left|(1-z)^{\alpha} f(z)-1\right| \leq|1-z|^{\alpha} \sum_{n=0}^{N}\left(h_{n}+h_{n}^{0}\right)|z|^{n}+\varepsilon \frac{|1-z|^{\alpha}}{(1-|z|)^{\alpha}},
$$

and the first term goes to 0 as $z \rightarrow 1$. As for the second, write $1-z=\rho e^{\mathrm{i} \theta}$ for $|\theta|<\pi-\phi$. For $\rho$ sufficiently close to 0 , we get that $|1-z| /(1-|z|) \leq \rho /\left(1-\left(1+\rho^{2}-2 \rho \cos (\pi-\phi)\right)^{1 / 2}\right)$, which admits a finite positive limit as $\rho \rightarrow 0$, allowing us to bound the right-hand side of the last displayed expression by a constant ( $\phi$-dependent) multiple of $\varepsilon$. Hence the result.

Now rewrite $G_{0}(s)$ as

$$
G_{0}(s)=2 \sum_{\mathfrak{m} \in \mathbf{M}_{g, 1}^{\geq 3}} \int \widehat{W N}_{\mathfrak{m}}(\mathrm{d} \mathbf{r}, \mathrm{d} \mathbf{x}) \bigotimes_{\mathfrak{e} \in E(\mathbf{m})} \mathrm{DS}_{r_{\mathfrak{e}}}\left(\frac{2 \tau^{\mathfrak{e}_{*}}}{\tau_{\mathfrak{l}}} s^{\tau_{\mathfrak{l}} / 2}\right) .
$$


We use the following trick: for a function $H$ on $\mathbf{M}_{g, 1}^{\geq 3}$ we have

$$
\sum_{\mathfrak{m} \in \mathbf{M}_{g, 1}^{\geq 3}} H(\mathfrak{m})=\sum_{\mathfrak{m}^{\circ} \in \mathbf{M}_{g, 1}^{\geq 3, \circ}}\left|\operatorname{Aut}\left(\mathfrak{m}^{\circ}\right)\right|^{-1} \sum_{\mathfrak{e}_{*} \in E\left(\mathfrak{m}^{\circ}\right)} H\left(\left(\mathfrak{m}^{\circ}, \mathfrak{e}_{*}\right)\right)
$$

where $\mathbf{M}_{g, 1}^{\geq 3, \circ}$ denotes unrooted maps and $\left(\mathfrak{m}^{\circ}, \mathfrak{e}_{*}\right)$ is the map $\mathfrak{m}^{\circ}$ rooted at $\mathfrak{e}_{*}$. Indeed, it holds that $\left(\mathfrak{m}^{\circ}, \mathfrak{e}\right)=\left(\mathfrak{m}^{\circ}, \mathfrak{e}^{\prime}\right)$ as a rooted map for edges $\mathfrak{e}, \mathfrak{e}^{\prime} \in E(\mathfrak{m})$ if and only if there exists an automorphism of $\mathfrak{m}$ sending $\mathfrak{e}$ to $\mathfrak{e}^{\prime}$. Thus, the orbits of the natural action of Aut $\left(\mathfrak{m}^{\circ}\right)$ on $E(\mathfrak{m})$ correspond to the distinct possible rootings of $\mathfrak{m}^{\circ}$. Moreover, if $p \cdot \mathfrak{e}=\mathfrak{e}$, then $p$ is the identity, which can be seen by reasoning in a step-by-step fashion from the edge $\mathfrak{e}$ and going to neighbors. Therefore, all the orbits have same cardinality $\left|\operatorname{Aut}\left(\mathfrak{m}^{\circ}\right)\right|$, and the claim follows. Applying it twice, once in each direction, allows us to get (with a straightforward definition for $\widehat{\mathrm{WN}}_{\mathfrak{m}^{\circ}}$ )

$$
\begin{aligned}
G_{0}(s) & =4 \sum_{\mathfrak{m}^{\circ} \in \mathbf{M}_{g, 1}^{\geq 3, \circ}}\left|\operatorname{Aut}\left(\mathfrak{m}^{\circ}\right)\right|^{-1} \int \widehat{\mathrm{WN}}_{\mathfrak{m}}^{\circ}(\mathrm{d} \mathbf{r}, \mathrm{d} \mathbf{x}) \bigotimes_{\mathfrak{e} \in E\left(\mathfrak{m}^{\circ}\right)} \mathrm{DS}_{r_{\mathfrak{e}}}\left(s^{\tau_{\mathfrak{l}} / 2}\right) \\
& =4 \sum_{\mathfrak{m} \in \mathbf{M}_{g, 1}^{\geq 3}}|E(\mathfrak{m})|^{-1} \int \widehat{W N}_{\mathfrak{m}}(\mathrm{d} \mathbf{r}, \mathrm{d} \mathbf{x}) \bigotimes_{\mathfrak{e} \in E(\mathfrak{m})} \operatorname{DS}_{r_{\mathfrak{e}}}\left(s^{\tau_{\mathfrak{l}} / 2}\right) .
\end{aligned}
$$

By (19) we have $\operatorname{DS}_{r}\left(s^{\tau(c)}\right)=\left(\left(1-\sqrt{1-s^{2}}\right) / s\right)^{r}$, so that we get $G_{0}(s)=G_{1} \circ G_{2}(s)$, where

$$
G_{1}(z)=4 \sum_{\mathfrak{m} \in \mathbf{M}_{g, 1}^{\geq 3}}|E(\mathfrak{m})|^{-1} \int \widehat{W N}_{\mathfrak{m}}(\mathrm{d} \mathbf{r}, \mathrm{d} \mathbf{x}) z^{\sum_{\mathbf{e} \in \mathbf{E}(\mathfrak{m})} r_{\mathbf{e}}},
$$

and

$$
G_{2}(s)=\mathrm{DS}_{2}\left(s^{\tau(c) / 2}\right)=\frac{2-s-2 \sqrt{1-s}}{s},
$$

which can be extended analytically to a domain $D_{R, \phi}$ with $R>1$ and $\phi \in(0, \pi / 2)$. Moreover, elementary computations show that $D_{R, \phi}$ is mapped to a domain $D_{1, \theta}$ for some $\theta \in(\pi / 2, \pi)$.

On the other hand, applying Lemma 8 to $G_{a}=1$ and $\rho_{a}=n=\left\lceil(2 a)^{1 / 2}\right\rceil$ gives that the $n$-th coefficient in $G_{1}$ satisfies

$$
\left[z^{n}\right] G_{1}(z) \sim 4\left(\frac{3}{2}\right)^{g} n^{5 g-4} \sum_{\mathfrak{m} \in \mathbf{M}_{g, 1}^{3}}|E(\mathfrak{m})|^{-1} \int_{\Delta_{E}} \frac{\lambda_{\Delta}(\mathrm{d} \mathbf{u})}{\sqrt{\mathcal{Z}^{\mathfrak{m}}(\mathbf{u})}}=C_{g}^{\prime \prime} n^{5 g-4} .
$$

Note that $|E(\mathfrak{m})|=2(6 g-3)$ for every term in the sum. From Lemma 12 , we deduce that $G_{1}(z) \sim \Gamma(5 g-3) C_{g}^{\prime \prime}(1-z)^{-(5 g-3)}$ for $z \rightarrow 1$ inside $D_{1, \theta}$. Combined with the fact that $G_{2}(s)=1+2 \sqrt{1-s}+O(1-s)$ as $s \rightarrow 1$ in $D_{R, \phi}$, we finally obtain that $G_{0}$ has an analytic continuation in $D_{R, \phi}$, and is equivalent to $2^{-5 g+3} \Gamma(5 g-3) C_{g}^{\prime \prime}(1-s)^{-(5 g-3) / 2}$ as $s \rightarrow 1$ inside this domain. An elementary transfer theorem [18, Corollary VI.1] allows us to conclude that $\left[s^{n}\right] G_{0}(s) \sim C_{g} n^{5(g-1) / 2}$, where the constant $C_{g}$ is computed as

$$
C_{g}=16\left(\frac{3}{64}\right)^{g} \frac{\Gamma(5 g-3)}{(6 g-3) \Gamma\left(\frac{5 g-3}{2}\right)} \sum_{\mathfrak{m} \in \mathbf{M}_{g, 1}^{3}} \int_{\Delta_{\mathbf{E}}} \frac{\lambda_{\Delta}(\mathrm{d} \mathbf{u})}{\sqrt{\mathcal{Z}^{\mathfrak{m}}(\mathbf{u})}} .
$$

Elementary (but tedious) computations give $C_{1}=1 / 24$, consistently with [4].

$4^{\text {e }}$ SÉRIE - TOME $42-2009-\mathrm{N}^{\mathrm{o}} 5$ 


\section{Remarks}

1. It is expected, and checked on numerous examples, see Gao [20], that "reasonable" families of random maps with size $n$ will have the universal property that their cardinality is equivalent to $C \alpha^{n} n^{-5 \chi(g) / 4}$ for some $C, \alpha$ depending on the model. A nice feature of the above approach is that, let aside the prefactor $16(3 / 64)^{g}$, it is reasonable to think that the gamma terms and summation over $\mathbf{M}_{g, 1}^{3}$ are not a particular feature of quadrangulations, and will appear for more general families of maps. However, it is extremely hard to determine the exact value of this sum as it involves a number of terms growing very quickly with $g$, and less and less tractable integrals.

2. In [10], the authors give an alternative expression for $C_{g}$, still in the form of a prefactor times a sum over trivalent maps with one face (and with an extra labeling of vertices). The expression of [10] is somewhat simpler than ours, as it does not involve integrals but only finite products of quantities that depend in an elementary way on the labeled map involved in the sum. It would be interesting to show directly that the two expressions are the same.

\section{Metrics on metric spaces}

In a metric space $(X, d)$, we let $B_{X}(x, r)$ (or simply $B(x, r)$ if there is no ambiguity) be the open ball centered at $x$ with radius $r$. We let $d_{H}$ be the usual Hausdorff distance between closed subsets of $X$

$$
d_{H}\left(C, C^{\prime}\right)=\inf \left\{\varepsilon>0: C \subseteq\left(C^{\prime}\right)^{\varepsilon} \text { and } C^{\prime} \subseteq C^{\varepsilon}\right\},
$$

where $A^{\varepsilon}=\left\{x \in X: \inf _{y \in A} d(x, y)<\varepsilon\right\}$ is the $\varepsilon$-neighborhood of $A$. We also let $d_{P}$ be the Prokhorov metric between Borel probability measures on $(X, d)$ defined by

$$
d_{P}\left(\mu, \mu^{\prime}\right)=\inf \left\{\varepsilon>0: \mu(C) \leq \mu^{\prime}\left(C^{\varepsilon}\right)+\varepsilon \text { for all closed } C\right\} .
$$

The topology associated with $d_{P}$ is that of weak convergence of probability measures on $X$. See [12, Chapter 11] for references.

\subsection{Gromov-Hausdorff distance}

We first recall the definition and first properties of the Gromov-Hausdorff distance, taken from $[9,16,22]$. If $\mathrm{X}=[X, d], \mathrm{X}^{\prime}=\left[X^{\prime}, d^{\prime}\right] \in \mathbb{M}$, we write

$$
\mathrm{d}_{\mathrm{GH}}\left(\mathrm{X}, \mathrm{X}^{\prime}\right)=\inf _{\phi, \phi^{\prime}} \delta_{H}\left(\phi(X), \phi^{\prime}\left(X^{\prime}\right)\right)
$$

where the infimum is taken over all isometries $\phi, \phi^{\prime}$ from $X, X^{\prime}$ into a common metric space $(Z, \delta)$.

An alternative useful description is given as follows. A correspondence between two sets $X, X^{\prime}$ is a subset $\mathcal{R} \subseteq X \times X^{\prime}$ such that $\pi(\mathcal{R})=X$ and $\pi^{\prime}(\mathcal{R})=X^{\prime}$, where $\pi: X \times X^{\prime} \rightarrow X, \pi^{\prime}: X \times X^{\prime} \rightarrow X^{\prime}$ are the canonical projections. We use $x \mathcal{R} x^{\prime}$ as an alternative notation for $\left(x, x^{\prime}\right) \in \mathcal{R}$, and we let $\mathcal{C}\left(X, X^{\prime}\right)$ be the set of correspondences between $X$ and $X^{\prime}$. If $(X, d),\left(X^{\prime}, d^{\prime}\right)$ are metric spaces, and $\mathcal{R}$ is a correspondence between $X$ and $X^{\prime}$, the distortion of $\mathcal{R}$ is defined as

$$
\operatorname{dis} \mathcal{R}=\sup \left\{\left|d(x, y)-d^{\prime}\left(x^{\prime}, y^{\prime}\right)\right|: x \mathcal{R} x^{\prime}, y \mathcal{R} y^{\prime}\right\} .
$$


Then

$$
\mathrm{d}_{\mathrm{GH}}\left(\mathrm{X}, \mathrm{X}^{\prime}\right)=\frac{1}{2} \inf _{\mathcal{R} \in \mathcal{C}\left(X, X^{\prime}\right)} \operatorname{dis} \mathcal{R}
$$

which of course does not depend on the particular representatives $X, X^{\prime}$ of $\mathrm{X}, \mathrm{X}^{\prime}$.

It is an easy exercise to check that $\operatorname{dis} \overline{\mathcal{R}}=\operatorname{dis} \mathcal{R}$ where $\overline{\mathcal{R}}$ is the closure of $\mathcal{R}$ in $X \times X^{\prime}$ for the product topology, so that the infimum in (22) could be taken over the set $\mathcal{C}_{c}\left(X, X^{\prime}\right)$ of compact correspondences (this set depends on the distances $d, d^{\prime}$ and not only on the sets $X, X^{\prime}$ ). The following properties [9, 16], combined with the forthcoming Proposition 8, will be useful in proving Theorem 2, in Section 7.1.

Proposition 5. - (i) The function $\mathrm{d}_{\mathrm{GH}}$ is a metric on $\mathbb{M}$. The space $\left(\mathbb{M}, \mathrm{d}_{\mathrm{GH}}\right)$ is separable and complete.

(ii) A subset $\mathbf{A} \subseteq \mathbb{M}$ is relatively compact if and only if the set $\{\operatorname{diam}(\mathrm{X}): \mathrm{X} \in \mathbf{A}\}$ is bounded, and for every $\varepsilon>0$, there exists $N>0$ such that every $\mathrm{X} \in \mathbf{A}$ can be covered with $N$ open balls of radius $\varepsilon$.

\subsection{Gromov-Hausdorff-Prokhorov metric}

Let $\mathrm{X}=[X, d, \mu], \mathrm{X}^{\prime}=\left[X^{\prime}, d^{\prime}, \mu^{\prime}\right] \in \mathbb{M}_{\mathrm{W}}$ We let

$$
\mathrm{d}_{\mathrm{GHP}}\left(\mathrm{X}, \mathrm{X}^{\prime}\right)=\inf _{\phi, \phi^{\prime}}\left(\delta_{H}\left(\phi(X), \phi^{\prime}\left(X^{\prime}\right)\right) \vee \delta_{P}\left(\phi_{*} \mu, \phi_{*}^{\prime} \mu^{\prime}\right)\right),
$$

where the infimum is taken over all isometries $\phi, \phi^{\prime}$ from $X, X^{\prime}$ into a metric space $(Z, \delta)$.

THEOREM 6. - The function $\mathrm{d}_{\mathrm{GHP}}$ defines a distance on $\mathbb{M}_{\mathrm{w}}$.

This result is well-known, see for instance [44, p. 762], with the slight difference that the supremum between $\delta_{H}$ and $\delta_{P}$ in the definition of $\mathrm{d}_{\mathrm{GHP}}$ is replaced by a sum. In order to make this article self-contained, we will provide a full proof of Theorem 6, to which the remaining part of this subsection is devoted. In order to handle the distance $\mathrm{d}_{\mathrm{GHP}}$, we develop an alternative definition with the help of correspondences and couplings. This is inspired in part from [21, 22].

If $\mu, \mu^{\prime}$ are Borel probability measures on $(X, d)$ and $\left(X^{\prime}, d^{\prime}\right)$, we say that a Borel measure $\nu$ on the product space $X \times X^{\prime}$ is a coupling between $\mu$ and $\mu^{\prime}$ if $\pi_{*} \nu=\mu$ and $\pi_{*}^{\prime} \nu=\mu^{\prime}$. We let $\mathcal{M}\left(\mu, \mu^{\prime}\right)$ be the set of couplings between $\mu$ and $\mu^{\prime}$. It is never empty as it contains the product measure $\mu \otimes \mu^{\prime}$, and is closed (and even compact since $X \times X^{\prime}$ is compact) for the weak topology.

A useful feature of couplings is the following. Since all spaces that are considered are Polish, it is known that we can write a coupling $\nu \in \mathcal{M}\left(\mu, \mu^{\prime}\right)$ in the form $\mu(\mathrm{d} x) Q_{\nu}(x, \mathrm{~d} y)$, where $Q_{\nu}$ is a Markov kernel from $X$ to $X^{\prime}$. In particular, if $\mu_{1}, \ldots, \mu_{k}$ are probability measures on the compact spaces $X_{1}, \ldots, X_{k}$ and $\nu_{i}, 1 \leq i \leq k-1$ are couplings between $\mu_{i}$ and $\mu_{i+1}$ respectively, we can define a Markov chain $\left(A_{1}, \ldots, A_{k}\right)$ such that $A_{i}$ is a random variable with distribution $\mu_{i}$ on $X_{i}$, with initial distribution $\mu_{1}$ and transition kernel $Q_{\nu_{i}}$ at step $i$. The joint law of $\left(A_{1}, A_{k}\right)$ is then denoted by $\nu_{1} \ldots \nu_{k}$ and is a coupling between $\mu_{1}$ and $\mu_{k}$.

$4^{\text {e }}$ SÉRIE - TOME $42-2009-\mathrm{N}^{\mathrm{o}} 5$ 
Proposition 6. - Let $\mathrm{X}=[X, d, \mu], \mathrm{X}^{\prime}=\left[X^{\prime}, d^{\prime}, \mu^{\prime}\right] \in \mathbb{M}_{\mathrm{w}}$. Then

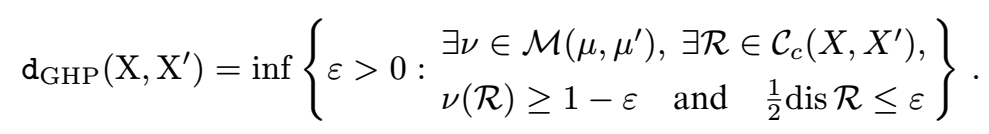

Proof. - Assume that $\mathrm{d}_{\mathrm{GHP}}\left(\mathrm{X}, \mathrm{X}^{\prime}\right)<\varepsilon$ and let $\phi, \phi^{\prime}$ be isometric embeddings of $X, X^{\prime}$ into $(Z, \delta)$ such that $\delta_{H}\left(\phi(X), \phi^{\prime}\left(X^{\prime}\right)\right)<\varepsilon$ and $\delta_{P}\left(\phi_{*} \mu, \phi_{*}^{\prime} \mu^{\prime}\right)<\varepsilon$. Arguing as in [9, Remark 7.3.12], we may and will assume that $(Z, \delta)$ is separable. Then the set $\mathcal{R}=\left\{\left(x, x^{\prime}\right) \in X \times X^{\prime}\right.$ : $\left.\delta\left(\phi(x), \phi^{\prime}\left(x^{\prime}\right)\right) \leq \varepsilon\right\}$ is an element of $\mathcal{C}_{c}\left(X, X^{\prime}\right)$. To evaluate its distortion, let $x \mathcal{R} x^{\prime}$ and $y \mathcal{R} y^{\prime}$, and write

$d(x, y)-d^{\prime}\left(x^{\prime}, y^{\prime}\right)=\delta(\phi(x), \phi(y))-\delta\left(\phi^{\prime}\left(x^{\prime}\right), \phi^{\prime}\left(y^{\prime}\right)\right) \leq \delta\left(\phi(x), \phi^{\prime}\left(x^{\prime}\right)\right)+\delta\left(\phi^{\prime}\left(y^{\prime}\right), \phi(y)\right) \leq 2 \varepsilon$,

and the lower bound is obtained in a similar way. Thus $\operatorname{dis} \mathcal{R} \leq 2 \varepsilon$.

Now, by a characterization of the Prokhorov distance (see [12, Corollary 11.6.4]) on separable metric spaces, we can find a coupling $\tilde{\nu} \in \mathcal{M}\left(\phi_{*} \mu, \phi_{*}^{\prime} \mu^{\prime}\right)$, which is a measure on $Z \times Z$, such that

$$
\tilde{\nu}(\{(x, y): \delta(x, y) \geq \varepsilon\}) \leq \varepsilon .
$$

We let $\nu_{1} \in \mathcal{M}\left(\mu, \phi_{*} \mu\right)$ be the image measure of $\mu$ by $x \mapsto(x, \phi(x))$ and $\nu_{2} \in \mathcal{M}\left(\phi_{*}^{\prime} \mu^{\prime}, \mu^{\prime}\right)$ be the image measure of $\mu^{\prime}$ by $x^{\prime} \mapsto\left(\phi^{\prime}\left(x^{\prime}\right), x^{\prime}\right)$. Then the coupling $\nu=\nu_{1} \tilde{\nu} \nu_{2}$ obviously satisfies $\nu\left(\mathcal{R}^{c}\right)=\tilde{\nu}(\{(x, y): \delta(x, y) \geq \varepsilon\}) \leq \varepsilon$ (here $\mathcal{R}^{c}$ is the complementary set of $\left.\mathcal{R}\right)$.

Conversely, assume given $\mathcal{R} \in \mathcal{C}_{c}\left(X, X^{\prime}\right)$ and $\nu \in \mathcal{M}\left(\mu, \mu^{\prime}\right)$ as in the right-hand side of (23) for the value $\varepsilon$. As in [9] we endow $X \sqcup X^{\prime}$ with a metric $\delta$ that is compatible with $d, d^{\prime}$, i.e. its restrictions to $X$ and $X^{\prime}$ coincide with $d, d^{\prime}$, and such that $\delta\left(x, x^{\prime}\right)=\varepsilon$ whenever $x \mathcal{R} x^{\prime}$. To do this, we set for every $x \in X, x^{\prime} \in X^{\prime}$

$$
\delta\left(x, x^{\prime}\right)=\inf \left\{d(x, y)+\varepsilon+d^{\prime}\left(y^{\prime}, x^{\prime}\right): y \mathcal{R} y^{\prime}\right\} .
$$

The only non-trivial point to show is the triangular inequality, and we prove only the hardest case, the others being symmetric or left as an exercise. Let $x, y \in X$ and $x^{\prime} \in X^{\prime}$, and consider $z \mathcal{R} z^{\prime}, t \mathcal{R} t^{\prime}$. Since by definition $\left|d(z, t)-d^{\prime}\left(z^{\prime}, t^{\prime}\right)\right| \leq 2 \varepsilon$ we estimate

$$
\begin{aligned}
& \left(d(x, z)+\varepsilon+d^{\prime}\left(z^{\prime}, x^{\prime}\right)\right)+\left(d(y, t)+\varepsilon+d^{\prime}\left(t^{\prime}, x^{\prime}\right)\right) \\
& \geq(d(x, z)+d(z, t)+d(y, t))+\left(d^{\prime}\left(z^{\prime}, x^{\prime}\right)+d^{\prime}\left(t^{\prime}, x^{\prime}\right)-d^{\prime}\left(z^{\prime}, t^{\prime}\right)\right) \\
& \geq d(x, y)+0,
\end{aligned}
$$

which is obtained by two applications of the triangular inequality in $X$ and $X^{\prime}$. Taking the infimum over $\left(z, z^{\prime}\right),\left(t, t^{\prime}\right)$ allows us to conclude that $d(x, y)=\delta(x, y) \leq \delta\left(x, x^{\prime}\right)+\delta\left(x^{\prime}, y\right)$.

Now $\nu$ induces a coupling $\tilde{\nu}$ on the disjoint union $X \sqcup X^{\prime}$ between $i_{*} \mu$ and $i_{*}^{\prime} \mu^{\prime}$, where $i, i^{\prime}$ are the canonical injections $X \rightarrow X \sqcup X^{\prime}$ and $X^{\prime} \rightarrow X \sqcup X^{\prime}$. Now obviously for every $a>1$

$$
\tilde{\nu}\left(\left\{\left(x, x^{\prime}\right): \delta\left(x, x^{\prime}\right) \geq a \varepsilon\right\}\right) \leq \nu\left(\mathcal{R}^{c}\right) \leq \varepsilon,
$$

so that $\delta_{P}\left(i_{*} \mu, i_{*}^{\prime} \mu^{\prime}\right) \leq \varepsilon$. Hence the result.

We are now ready to prove that $\mathrm{d}_{\mathrm{GHP}}$ is indeed a distance. Symmetry is obvious, as well as $\mathrm{d}_{\mathrm{GHP}}(\mathrm{X}, \mathrm{X})=0$. 
Separation. Let $\mathrm{X}=[X, d, \mu], \mathrm{X}^{\prime}=\left[X^{\prime}, d^{\prime}, \mu^{\prime}\right] \in \mathbb{M}_{\mathrm{w}}$, with $\mathrm{d}_{\mathrm{GHP}}\left(\mathrm{X}, \mathrm{X}^{\prime}\right)=0$. Then for every $n \geq 1$ we can find $\mathcal{R}_{n} \in \mathcal{C}_{c}\left(X, X^{\prime}\right)$ and $\nu_{n} \in \mathcal{M}\left(\mu, \mu^{\prime}\right)$ with dis $\mathcal{R}_{n} \leq 2^{-n}$ and $\nu_{n}\left(\mathcal{R}_{n}^{c}\right) \leq 2^{-n}$.

Endow the product space $X \times X^{\prime}$ with the distance $\delta\left(\left(x, x^{\prime}\right),\left(y, y^{\prime}\right)\right)=\max \left(d(x, y), d^{\prime}\left(x^{\prime}, y^{\prime}\right)\right)$. Then $\left(X \times X^{\prime}, \delta\right)$ is a compact metric space, so that the set of compact subsets of $X \times X^{\prime}$, endowed with the Hausdorff distance $\delta_{H}$, is compact [9, Theorem 7.3.8]. Therefore, $\left(\mathcal{R}_{n}, n \geq 1\right)$ converges up to extraction to some compact $\mathcal{R}$. It is immediate to check that the latter is an element of $\mathcal{C}_{c}\left(X, X^{\prime}\right)$, with dis $\mathcal{R}=0$, and this implies that $\mathcal{R}$ is the graph of a bijective isometry $h: X \rightarrow X^{\prime}$.

On the other hand, since $\mathcal{M}\left(\mu, \mu^{\prime}\right)$ is compact for the weak topology, the couplings $\left(\nu_{n}, n \geq 1\right)$ converge weakly, up to re-extracting, to some $\nu \in \mathcal{M}\left(\mu, \mu^{\prime}\right)$. Since $\mathcal{R}_{n}$ converges to $\mathcal{R}$ for the distance $\delta_{H}$, it holds that for every $\varepsilon>0, \mathcal{R}_{n} \subseteq \overline{\mathcal{R}}^{\varepsilon}=\left\{\left(x, x^{\prime}\right)\right.$ : $\left.\inf _{\left(y, y^{\prime}\right) \in \mathcal{R}} \delta\left(\left(x, x^{\prime}\right),\left(y, y^{\prime}\right)\right) \leq \varepsilon\right\}$ for every $n$ large enough. For such $n$, we have $\nu_{n}\left(\overline{\mathcal{R}}^{\varepsilon}\right) \geq \nu_{n}\left(\mathcal{R}_{n}\right) \geq 1-2^{-n}$, and by well-known properties of weak convergence of probability measures, since $\overline{\mathcal{R}}^{\varepsilon}$ is closed, this entails that $\nu\left(\overline{\mathcal{R}}^{\varepsilon}\right) \geq \lim \sup \nu_{n}\left(\overline{\mathcal{R}}^{\varepsilon}\right)=1$. Letting $\varepsilon \rightarrow 0$ shows that $\nu(\mathcal{R})=1$, and we conclude that $\nu$ is supported on the graph of the isometry $h$. Thus, for every measurable $g: X^{\prime} \rightarrow \mathbb{R}_{+}$,

$\int_{X} g(h(x)) \mu(\mathrm{d} x)=\int_{X \times X^{\prime}} g(h(x)) \nu\left(\mathrm{d} x, \mathrm{~d} x^{\prime}\right)=\int_{X \times X^{\prime}} g\left(x^{\prime}\right) \nu\left(\mathrm{d} x, \mathrm{~d} x^{\prime}\right)=\int_{X^{\prime}} g\left(x^{\prime}\right) \mu^{\prime}\left(\mathrm{d} x^{\prime}\right)$,

so $\mu^{\prime}=h_{*} \mu$. Since $X^{\prime}=h(X)$, we obtain $\mathrm{X}=\mathrm{X}^{\prime}$.

Triangular inequality. Let $\mathrm{X}=[X, d, \mu], \mathrm{X}^{\prime}=\left[X^{\prime}, d^{\prime}, \mu^{\prime}\right], \mathrm{X}^{\prime \prime}=\left[X^{\prime \prime}, d^{\prime \prime}, \mu^{\prime \prime}\right] \in \mathbb{M}_{\mathrm{w}}$, and let us assume $\mathrm{d}_{\mathrm{GHP}}\left(\mathrm{X}, \mathrm{X}^{\prime}\right)<\varepsilon_{1}$ and $\mathrm{d}_{\mathrm{GHP}}\left(\mathrm{X}^{\prime}, \mathrm{X}^{\prime \prime}\right)<\varepsilon_{2}$. We wish to show that $\mathrm{d}_{\mathrm{GHP}}\left(\mathrm{X}, \mathrm{X}^{\prime \prime}\right) \leq \varepsilon_{1}+\varepsilon_{2}$. By Proposition 6, we can find $\mathcal{R}_{1} \in \mathcal{C}_{c}\left(X, X^{\prime}\right), \mathcal{R}_{2} \in \mathcal{C}_{c}\left(X^{\prime}, X^{\prime \prime}\right)$ and $\nu_{1} \in \mathcal{M}\left(\mu, \mu^{\prime}\right), \nu_{2} \in \mathcal{M}\left(\mu^{\prime}, \mu^{\prime \prime}\right)$ such that dis $\mathcal{R}_{i} \leq 2 \varepsilon_{i}$ and $\nu_{i}\left(\mathcal{R}_{i}^{c}\right) \leq \varepsilon_{i}, i \in\{1,2\}$.

Let $\nu_{3}=\nu_{1} \nu_{2}$ and define a correspondence $\mathcal{R}_{3} \in \mathcal{C}\left(X, X^{\prime \prime}\right)$ by $x \mathcal{R}_{3} x^{\prime \prime}$ if and only if there exists $x^{\prime} \in X^{\prime}$ such that $x \mathcal{R}_{1} x^{\prime}$ and $x^{\prime} \mathcal{R}_{2} x^{\prime \prime}$, that is $\mathcal{R}_{3}$ is the image by the (continuous) canonical projection $X \times X^{\prime} \times X^{\prime \prime} \rightarrow X \times X^{\prime}$ of the compact set $\left(\mathcal{R}_{1} \times X^{\prime \prime}\right) \cap\left(X \times \mathcal{R}_{2}\right)$, and hence is compact. To evaluate the distortion, let $x \mathcal{R}_{1} x^{\prime}, x^{\prime} \mathcal{R}_{2} x^{\prime \prime}$ and $y \mathcal{R}_{1} y^{\prime}, y^{\prime} \mathcal{R}_{2} y^{\prime \prime}$ and write

$$
\left|d(x, y)-d^{\prime \prime}\left(x^{\prime \prime}, y^{\prime \prime}\right)\right| \leq\left|d(x, y)-d^{\prime}\left(x^{\prime}, y^{\prime}\right)\right|+\left|d^{\prime}\left(x^{\prime}, y^{\prime}\right)-d^{\prime \prime}\left(x^{\prime \prime}, y^{\prime \prime}\right)\right| \leq 2\left(\varepsilon_{1}+\varepsilon_{2}\right),
$$

so that dis $\mathcal{R}_{3} \leq 2\left(\varepsilon_{1}+\varepsilon_{2}\right)$.

Also note that $\mathbb{1}_{\left(\mathcal{R}_{3}\right)^{c}}\left(x, x^{\prime \prime}\right) \leq \mathbb{1}_{\mathcal{R}_{1}^{c}}\left(x, x^{\prime}\right)+\mathbb{1}_{\mathcal{R}_{2}^{c}}\left(x^{\prime}, x^{\prime \prime}\right)$ for every $\left(x, x^{\prime}, x^{\prime \prime}\right) \in X \times X^{\prime} \times X^{\prime \prime}$. Indeed, if $\left(x, x^{\prime \prime}\right) \notin \mathcal{R}_{3}$, then for all $x^{\prime} \in X^{\prime}$, either $\left(x, x^{\prime}\right) \notin \mathcal{R}_{1}$ or $\left(x^{\prime}, x^{\prime \prime}\right) \notin \mathcal{R}_{2}$. We deduce

$$
\begin{aligned}
\nu_{3}\left(\mathcal{R}_{3}^{c}\right) \leq & \int_{X \times X^{\prime} \times X^{\prime \prime}} \mu(\mathrm{d} x) Q_{1}\left(x, \mathrm{~d} x^{\prime}\right) Q_{2}\left(x^{\prime}, \mathrm{d} x^{\prime \prime}\right) \mathbb{1}_{\mathcal{R}_{1}^{c}}\left(x, x^{\prime}\right) \\
& +\int_{X \times X^{\prime} \times X^{\prime \prime}} \mu(\mathrm{d} x) Q_{1}\left(x, \mathrm{~d} x^{\prime}\right) Q_{2}\left(x^{\prime}, \mathrm{d} x^{\prime \prime}\right) \mathbb{1}_{\mathcal{R}_{2}^{c}}\left(x^{\prime}, x^{\prime \prime}\right) \\
= & \nu_{1}\left(\mathcal{R}_{1}^{c}\right)+\nu_{2}\left(\mathcal{R}_{2}^{c}\right) \leq \varepsilon_{1}+\varepsilon_{2} .
\end{aligned}
$$

This is the wanted result, concluding the proof of Theorem 6 . 


\subsection{Relation with other metrics}

It turns out that the topology determined by $\mathrm{d}_{\mathrm{GHP}}$ has already been considered by [17, 19], where a different distance was introduced. Note that in [17], the authors restricted their attention to compact spaces which are $\mathbb{R}$-trees, but all of their results up to Theorem 2.5 in this reference can be rewritten for the space $\mathbb{M}_{\mathrm{w}}$ without any change.

An $\varepsilon$-isometry from $X$ to $X^{\prime}$ is a mapping $f: X \rightarrow X^{\prime}$ such that $(f(X))^{\varepsilon}=X^{\prime}$ (we say that $f(X)$ is an $\varepsilon$-net in $X^{\prime}$ ) and for every $x, y \in X$,

$$
\left|d(x, y)-d^{\prime}(f(x), f(y))\right| \leq \varepsilon .
$$

We say that $\mathrm{X}=[X, d, \mu], \mathrm{X}^{\prime}=\left[X^{\prime}, d^{\prime}, \mu^{\prime}\right] \in \mathbb{M}_{\mathrm{w}}$ are $\varepsilon$-close if there exist two measurable $\varepsilon$-isometries $f: X \rightarrow X^{\prime}$ and $g: X^{\prime} \rightarrow X$ such that $d_{P}^{\prime}\left(f_{*} \mu, \mu^{\prime}\right) \vee d_{P}\left(g_{*} \mu^{\prime}, \mu\right) \leq \varepsilon$. Obviously, being $\varepsilon$-close is indeed an isometry class property and does not depend on the choice of representatives.

We define $\Delta_{\mathrm{GHP}}\left(\mathrm{X}, \mathrm{X}^{\prime}\right)$ as the infimal $\varepsilon$ such that $\mathrm{X}, \mathrm{X}^{\prime}$ are $\varepsilon$-close. This does not define a true distance, as the triangle inequality is satisfied only up to a factor of 2 . This quasidistance is however separated and thus sufficient to define a separated topology on $\mathbb{M}_{\mathrm{w}}$. It is also fully discussed in [17], building on metrization theorems for uniform spaces [6], how to build a true distance using $\Delta_{\mathrm{GHP}}$, which is yet different from $\mathrm{d}_{\mathrm{GHP}}$.

Proposition 7. - It holds that $3^{-1} \Delta_{\mathrm{GHP}} \leq \mathrm{d}_{\mathrm{GHP}} \leq 2 \Delta_{\mathrm{GHP}}$.

Proof. - Assume that $\mathrm{d}_{\mathrm{GHP}}\left(\mathrm{X}, \mathrm{X}^{\prime}\right)<\varepsilon$, and let $\mathcal{R}, \nu$ be a correspondence and a coupling between $\mathrm{X}$ and $\mathrm{X}^{\prime}$ such that dis $\mathcal{R} \leq 2 \varepsilon$ and $\nu(\mathcal{R}) \geq 1-\varepsilon$. We build a measurable $3 \varepsilon$-isometry $f: X \rightarrow X^{\prime}$ out of $\mathcal{R}$, by taking $\left\{x_{1}, \ldots, x_{N}\right\}$ a minimal $\varepsilon$-net in $X$, choosing for every $i$ some $x_{i}^{\prime}$ such that $x_{i} \mathcal{R} x_{i}^{\prime}$, and finally defining $f\left(B_{i}\right)=\left\{x_{i}^{\prime}\right\}$ where $B_{i}=B\left(x_{i}, \varepsilon\right) \backslash \bigcup_{1 \leq j \leq i-1} B\left(x_{j}, \varepsilon\right)$. Then the image measure $\tilde{\nu}$ of $\nu$ by $\left(x, x^{\prime}\right) \mapsto\left(f(x), x^{\prime}\right)$ is a coupling between $f_{*} \mu$ and $\mu^{\prime}$. Moreover, from the way $f$ is constructed, if $d^{\prime}\left(f(x), x^{\prime}\right)>3 \varepsilon$ then $\left(x, x^{\prime}\right) \notin \mathcal{R}$, so that $\tilde{\nu}\left\{\left(x^{\prime}, y^{\prime}\right): d^{\prime}\left(x^{\prime}, y^{\prime}\right)>3 \varepsilon\right\} \leq \nu\left(\mathcal{R}^{c}\right) \leq \varepsilon$. Thus $d_{P}^{\prime}\left(f_{*} \mu, \mu^{\prime}\right) \leq 3 \varepsilon$, and by a symmetrical argument we conclude that $\mathrm{X}$ and $\mathrm{X}^{\prime}$ are $3 \varepsilon$-close.

Conversely assume given $f: X \rightarrow X^{\prime}$ an $\varepsilon$-isometry with $d_{P}^{\prime}\left(f_{*} \mu, \mu^{\prime}\right) \leq \varepsilon$. Define a correspondence between $X$ and $X^{\prime}$ by $x \mathcal{R} x^{\prime}$ if $d^{\prime}\left(x^{\prime}, f(x)\right) \leq \varepsilon$. Its distortion is estimated as follows:

$$
\begin{aligned}
& \left|d(x, y)-d^{\prime}\left(x^{\prime}, y^{\prime}\right)\right| \\
& \quad \leq|d(x, y)-d(f(x), f(y))|+\left|d(f(x), f(y))-d\left(f(x), y^{\prime}\right)\right|+\left|d\left(f(x), y^{\prime}\right)-d\left(x^{\prime}, y^{\prime}\right)\right|,
\end{aligned}
$$

which is less than $4 \varepsilon$ whenever $x \mathcal{R} x^{\prime}, y \mathcal{R} y^{\prime}$. We take the closure of $\mathcal{R}$, which has same distortion, and still call it $\mathcal{R}$. Take a coupling $\tilde{\nu}$ between $f_{*} \mu$ and $\mu^{\prime}$ with $\tilde{\nu}\left\{\left(x^{\prime}, y^{\prime}\right): d^{\prime}\left(x^{\prime}, y^{\prime}\right) \geq \varepsilon\right\} \leq \varepsilon$ and let $\nu_{1}$ be the image measure of $\mu$ by $x \mapsto(x, f(x))$. Then $\nu=\nu_{1} \tilde{\nu}$ satisfies $\nu(\mathcal{R}) \geq 1-\varepsilon$. We conclude that $\mathrm{d}_{\mathrm{GHP}}\left(\mathrm{X}, \mathrm{X}^{\prime}\right) \leq 2 \varepsilon$.

As a consequence of this and [17, Lemma 2.3], we obtain that the metric properties of Evans and Winter's distance are the same as those of $\mathrm{d}_{\mathrm{GHP}}$. In particular:

Proposition 8. - (i) The metric space $\left(\mathbb{M}_{\mathrm{w}}, \mathrm{d}_{\mathrm{GHP}}\right)$ is separable and complete.

(ii) A subset $\mathbf{A}$ of $\mathbb{M}_{\mathrm{w}}$ is relatively compact if and only if the subset $\{[X, d]:[X, d, \mu] \in \mathbf{A}\}$ is relatively compact in $\left(\mathbb{M}, \mathrm{d}_{\mathrm{GH}}\right)$. 


\subsection{Marked topologies}

In this section we introduce a marked variant of the Gromov-Hausdorff topology.

A $k$-marked metric space is a triple $\left(X, d,\left(C_{1}, \ldots, C_{k}\right)\right)$ where $(X, d)$ is a compact metric space and $C_{1}, \ldots, C_{k}$ are compact subsets of $X$. The isometry classes of marked spaces $\left[X, d,\left(C_{1}, \ldots, C_{k}\right)\right]$ are defined in the obvious way, and their set is denoted by $\mathbb{M}_{k}^{*}$. We let, with obvious notations,

$$
\mathrm{d}_{\mathrm{GH}}^{k}\left(\mathrm{X}, \mathrm{X}^{\prime}\right)=\inf _{\phi, \phi^{\prime}}\left(\delta_{H}\left(\phi(X), \phi^{\prime}\left(X^{\prime}\right)\right) \vee \max _{1 \leq i \leq k} \delta_{H}\left(\phi\left(C_{i}\right), \phi^{\prime}\left(C_{i}^{\prime}\right)\right)\right),
$$

the infimum being taken over all isometric embeddings $\phi, \phi^{\prime}$ of $\mathrm{X}, \mathrm{X}^{\prime}$ into a common metric space $(Z, \delta)$.

The following statement is a straightforward generalization of the classical results of Proposition 5, and its proof is left as an exercise to the reader.

Proposition 9. - (i) The function $\mathrm{d}_{\mathrm{GH}}^{k}$ is a distance on $\mathbb{M}_{k}^{*}$, which is alternatively described by

$$
\mathrm{d}_{\mathrm{GH}}^{k}\left(\mathrm{X}, \mathrm{X}^{\prime}\right)=\frac{1}{2} \inf _{\mathcal{R}} \operatorname{dis} \mathcal{R},
$$

the infimum being taken over correspondences $\mathcal{R} \in \mathcal{C}_{c}\left(X, X^{\prime}\right)$ such that $\mathcal{R} \cap\left(C_{i} \times C_{i}^{\prime}\right) \in \mathcal{C}\left(C_{i}, C_{i}^{\prime}\right)$.

(ii) The metric space $\left(\mathbb{M}_{k}^{*}, \mathrm{~d}_{\mathrm{GH}}^{k}\right)$ is separable and complete.

(iii) A subset $\mathbf{A} \subseteq \mathbb{M}_{k}^{*}$ is relatively compact if and only if $\left\{[X, d]:\left[X, d,\left(C_{1}, \ldots, C_{k}\right)\right] \in A\right\}$ is relatively compact in $\left(\mathbb{M}, \mathrm{d}_{\mathrm{GH}}\right)$.

When $C_{1}, \ldots, C_{k}$ are singletons, we write for notational convenience $(X, d, \mathbf{x})$ instead of $\left(X, d,\left(\left\{x_{1}\right\}, \ldots,\left\{x_{k}\right\}\right)\right)$. The reader will easily be convinced that the subspace of such marked spaces is closed in $\mathbb{M}_{k}^{*}$.

\subsection{Randomly marked spaces}

We now introduce random marks on metric spaces. If $\mathrm{X}=[X, d, \mu] \in \mathbb{M}_{\mathrm{w}}$, we can consider an i.i.d. sequence $\left(U_{1}, \ldots, U_{k}\right)$ which is $\mu$-distributed in $X$, and we want to consider the isometry class of $\left[X, d,\left(U_{1}, \ldots, U_{k}\right)\right]$ as a random variable in $\mathbb{M}_{k}^{*}$. We also wish to consider randomly marked versions of spaces that are themselves random. To do this implies that we check a couple of measurability issues. We introduce for each $k$ a kernel from $\mathbb{M}_{\mathrm{w}}$ to $\mathbb{M}_{k}^{*}$, defined as follows:

$$
\mathrm{m}_{k}(\mathrm{X}, \mathbf{A})=\int_{X^{k}} \mu^{\otimes k}(\mathrm{~d} \mathbf{x}) \mathbb{1}_{\mathbf{A}}([X, d, \mathbf{x}]), \quad \mathrm{X}=[X, d, \mu] \in \mathbb{M}_{\mathrm{w}}, \mathbf{A} \in \mathcal{B}\left(\mathbb{M}_{k}^{*}\right) .
$$

Note that the integral makes sense as the function $X^{k} \rightarrow \mathbb{M}_{k}^{*}$ which maps $\mathbf{x}$ to $[X, d, \mathbf{x}]$ is obviously continuous, so that $\mathbf{x} \mapsto \mathbb{1}_{\mathbf{A}}([X, d, \mathbf{x}])$ is measurable by composition. Also notice that it is independent of the particular choice of the representative $(X, d, \mu) \in \mathrm{X}$, and hence is unambiguous.

Lemma 13. - The formula (24) defines a Markov kernel. 
Proof. - It is obvious from the definition that for every $\mathrm{X}$, the function $\mathbf{A} \mapsto \mathrm{m}_{k}(\mathrm{X}, \mathbf{A})$ defines a Borel measure on $\mathbb{M}_{k}^{*}$.

Now let $\mathbf{A}$ be closed in $\mathbb{M}_{k}^{*}$. We show $\mathrm{X} \mapsto \mathrm{m}_{k}(\mathrm{X}, \mathbf{A})$ is upper-semicontinuous. Taking $\varepsilon>0$, for any $\mathrm{X}^{\prime} \in \mathbb{M}_{\mathrm{w}}$ with $\mathrm{d}_{\mathrm{GHP}}\left(\mathrm{X}, \mathrm{X}^{\prime}\right)<\varepsilon$, and with obvious notations, we can find $\mathcal{R} \in \mathcal{C}_{c}\left(X, X^{\prime}\right)$ and $\nu \in \mathcal{M}\left(\mu, \mu^{\prime}\right)$ such that $\nu(\mathcal{R})>1-\varepsilon$ and dis $\mathcal{R}<2 \varepsilon$. We write

$$
\begin{aligned}
& \int_{X^{k}} \mu^{\otimes k}(\mathrm{~d} \mathbf{x})\left(1-\mathbb{1}_{\mathbf{A}^{\varepsilon}}([X, d, \mathbf{x}])\right)=\int_{\left(X \times X^{\prime}\right)^{k}} \nu^{\otimes k}\left(\mathrm{~d} \mathbf{x}, \mathrm{d} \mathbf{x}^{\prime}\right)\left(1-\mathbb{1}_{\mathbf{A}^{\varepsilon}}([X, d, \mathbf{x}])\right) \\
& \leq 1-(1-\varepsilon)^{k}+\int_{\left(X \times X^{\prime}\right)^{k}} \nu^{\otimes k}\left(\mathrm{~d} \mathbf{x}, \mathrm{d} \mathbf{x}^{\prime}\right) \mathbb{1}_{\left\{x_{i} \mathcal{R} x_{i}^{\prime}, 1 \leq i \leq k\right\}}\left(1-\mathbb{1}_{\mathbf{A}^{\varepsilon}}([X, d, \mathbf{x}])\right) \\
& \leq 1-(1-\varepsilon)^{k}+\int_{\left(X^{\prime}\right)^{k}} \mathrm{~d}\left(\mu^{\prime}\right)^{\otimes k}\left(\mathbf{x}^{\prime}\right)\left(1-\mathbb{1}_{\mathbf{A}}\left(\left[X^{\prime}, d^{\prime}, \mathbf{x}^{\prime}\right]\right)\right)
\end{aligned}
$$

where we used the fact that $\nu^{\otimes k}\left(\left\{\left(\mathbf{x}, \mathbf{x}^{\prime}\right): x_{i} \mathcal{R} x_{i}^{\prime}, 1 \leq i \leq k\right\}\right) \geq(1-\varepsilon)^{k}$ and that whenever the latter event occurs, it holds that $\mathrm{d}_{\mathrm{GH}}^{k}\left([X, d, \mathbf{x}],\left[X^{\prime}, d^{\prime}, \mathbf{x}^{\prime}\right]\right)<\varepsilon$. Since $\mathbf{A}^{\varepsilon}$ decreases to $\mathbf{A}$ as $\varepsilon \downarrow 0$ we see that the left-hand side can be made arbitrarily close to $1-\mathrm{m}_{k}(\mathrm{X}, \mathbf{A})$ provided $\varepsilon$ is sufficiently small. Therefore

$$
\limsup _{\mathrm{X}^{\prime} \rightarrow \mathrm{X}} \mathrm{m}_{k}\left(\mathrm{X}^{\prime}, \mathbf{A}\right) \leq \mathrm{m}_{k}(\mathrm{X}, \mathbf{A})
$$

as claimed. We conclude by a monotone class argument that $\mathrm{m}_{k}(\cdot, \mathbf{A})$ is measurable for all Borel $\mathbf{A} \subseteq \mathbb{M}_{k}^{*}$.

A random variable with law $\mathrm{m}_{k}(\mathrm{X}, \cdot)$ can be considered as the rigorous definition of the space $\mathrm{X}$ randomly marked with $k$ independent $\mu$-chosen points. If $\mathbf{M}(\mathrm{dX})$ is a distribution on $\mathbb{M}_{\mathrm{w}}$, a random variable with law $\mathbf{M}(\mathrm{dX}) \mathrm{m}_{k}(\mathrm{X}, \cdot)$ is interpreted as a weighted space chosen according to $\mathbf{M}$, and then marked randomly using the weight.

A key property of randomly marked spaces is the following. If $\mathrm{X} \in \mathbb{M}_{\mathrm{w}}$, we let $\mathrm{X}^{k}$ be the canonical random variable with law $\mathrm{m}_{k}(\mathrm{X}, \cdot)$. A similar notation holds if $\mathrm{X}$ is a random variable with values in $\mathbb{M}_{\mathrm{w}}$.

Proposition 10. - Let $\left(\mathrm{X}_{n}, n \geq 1\right)$ be random variables in $\mathbb{M}_{\mathrm{w}}$ converging to $\mathrm{X}$ in distribution. Then the randomly marked spaces $\mathrm{X}_{n}^{k}$ converge in distribution towards $\mathrm{X}^{k}$, for the topology of $\mathbb{M}_{k}^{*}$.

Proof. - Using the Skorokhod representation theorem, we may assume that the probability space $(\Omega, \mathcal{F}, P)$ on which the random variables are defined is such that the convergence $\mathrm{X}_{n} \rightarrow \mathrm{X}$ holds a.s. Now, we have from the proof of Lemma 13 that $\mathrm{m}_{k}(\cdot, \mathbf{A})$ is upper-semicontinuous for every closed $\mathbf{A} \subseteq \mathbb{M}_{k}^{*}$. By the reversed Fatou lemma this gives

$$
\limsup _{n \rightarrow \infty} E\left[\mathrm{~m}_{k}\left(\mathrm{X}_{n}, \mathbf{A}\right)\right] \leq E\left[\mathrm{~m}_{k}(\mathrm{X}, \mathbf{A})\right]
$$

which says that $\lim \sup P\left(\mathrm{X}_{n}^{k} \in \mathbf{A}\right) \leq P\left(\mathrm{X}^{k} \in \mathbf{A}\right)$, and the conclusion follows from a wellknown characterization of weak convergence.

\subsection{Three lemmas}

We end this section with key lemmas to the proof of Theorem 3. 


\subsubsection{Diffuse measures}

Lemma 14. - Let $\left[X_{n}, d_{n}, \mu_{n}\right], n \geq 1$ converge in $\mathbb{M}_{\mathrm{w}}$ towards $[X, d, \mu]$. Assume that

$$
\lim _{\varepsilon \downarrow 0} \liminf _{n \rightarrow \infty} \int_{X_{n}} \mu_{n}(\mathrm{~d} x) \mu_{n}(B(x, \varepsilon))=0 .
$$

Then $\mu$ is a diffuse measure.

Proof. - From the proof of Lemma 13, we know that if $\mathbf{A}$ is an open set in $\mathbb{M}_{2}^{*}$, then $\mathrm{X} \mapsto \mathrm{m}_{2}(\mathrm{X}, \mathbf{A})$ is lower-semicontinuous from $\mathbb{M}_{\mathrm{w}}$ to $\mathbb{M}_{2}^{*}$. Now write

$$
\int_{X_{n}} \mu_{n}(\mathrm{~d} x) \mu_{n}(B(x, \varepsilon))=\int_{X_{n}^{2}} \mu_{n}^{\otimes 2}(\mathrm{~d} x, \mathrm{~d} y) \mathbb{1}_{\left\{d_{n}(x, y)<\varepsilon\right\}}=\mathrm{m}_{2}\left(\mathrm{X}_{n}, \mathbf{A}\right)
$$

where $\mathbf{A}=\left\{\mathrm{X}^{\prime}=\left[X^{\prime}, d^{\prime},\left(x^{\prime}, y^{\prime}\right)\right] \in \mathbb{M}_{2}^{*}: d^{\prime}\left(x^{\prime}, y^{\prime}\right)<\varepsilon\right\}$ is open. Therefore, it holds that

$$
\liminf _{n \rightarrow \infty} \int_{X_{n}} \mu_{n}(\mathrm{~d} x) \mu_{n}(B(x, \varepsilon)) \geq \int_{X} \mu(\mathrm{d} x) \mu(B(x, \varepsilon)) .
$$

Letting $\varepsilon \rightarrow 0$ and using the hypothesis give the result.

\subsubsection{Support}

Lemma 15. - Let $\left[X_{n}, d_{n}, \mu_{n}\right], n \geq 1$ be a sequence converging to $[X, d, \mu]$ in $\mathbb{M}_{\mathrm{w}}$. Let $Y_{n} \subseteq X_{n}$ be $\varepsilon_{n}$-dense in $X_{n}$, for some $\varepsilon_{n} \rightarrow 0$ (i.e. every point of $X_{n}$ is at distance at most $\varepsilon_{n}$ of a point of $\left.Y_{n}\right)$. Assume that for every $\varepsilon>0$,

$$
\limsup _{n \rightarrow \infty} \inf _{x \in Y_{n}} \mu_{n}(B(x, \varepsilon))>0 .
$$

Then $\operatorname{supp} \mu=X$.

Proof. - Take $x \in X$ and $\varepsilon>0$. We must show that $\mu(B(x, \varepsilon))>0$. To this end take compact correspondences $\mathcal{R}_{n}$ between $X_{n}, X$ with distortion going to 0 , and couplings $\nu_{n}$ between $\mu_{n}$ and $\mu$ with $\nu_{n}\left(\mathcal{R}_{n}\right) \rightarrow 1$. Let $x_{n}$ be such that $x_{n} \mathcal{R}_{n} x$ and $x_{n}^{\prime} \in Y_{n}$ be at distance $\leq \varepsilon_{n}$ from $x_{n}$. Then

$$
\mu(B(x, \varepsilon))=\int_{X} \mu(\mathrm{d} y) \mathbb{1}_{\{d(x, y)<\varepsilon\}} \geq \int_{X_{n} \times X} \nu_{n}\left(\mathrm{~d} y_{n}, \mathrm{~d} y\right) \mathbb{1}_{\{d(x, y)<\varepsilon\}} .
$$

Now take $n$ large enough so that dis $\mathcal{R}_{n}<\varepsilon / 2-\varepsilon_{n}$. Then $d_{n}\left(x_{n}^{\prime}, y_{n}\right)<\varepsilon / 2$ implies $d(x, y)<\varepsilon$ as soon as $y_{n} \mathcal{R}_{n} y$. Therefore the last integral is greater than or equal to

$$
\begin{aligned}
\int_{\mathcal{R}_{n}} \nu_{n}\left(\mathrm{~d} y_{n}, \mathrm{~d} y\right) \mathbb{1}_{\left\{d_{n}\left(x_{n}^{\prime}, y_{n}\right)<\varepsilon / 2\right\}} & \geq \int_{X_{n} \times X} \nu_{n}\left(\mathrm{~d} y_{n}, \mathrm{~d} y\right) \mathbb{1}_{\left\{d_{n}\left(x_{n}^{\prime}, y_{n}\right)<\varepsilon / 2\right\}}-\nu_{n}\left(\mathcal{R}_{n}^{c}\right) \\
& =\mu_{n}\left(B\left(x_{n}^{\prime}, \varepsilon / 2\right)\right)-\nu_{n}\left(\mathcal{R}_{n}^{c}\right) .
\end{aligned}
$$

This is bounded below by $\inf _{x \in Y_{n}} \mu_{n}(B(x, \varepsilon / 2))-\nu_{n}\left(\mathcal{R}_{n}^{c}\right)$, which has positive lim sup as $n \rightarrow \infty$. Hence the result.

$4^{\text {e }}$ SÉRIE - TOME $42-2009-\mathrm{N}^{\mathrm{o}} 5$ 
6.6.3. D-median points in geodesic metric spaces. - Since compact geodesic metric spaces form a closed subset $\mathrm{PM}$ of $\left(\mathbb{M}, \mathrm{d}_{\mathrm{GH}}\right)\left(\left[9\right.\right.$, Thorem 7.5.1]), it is immediate that the set $\mathrm{PM}_{\mathrm{w}}$ of spaces $[X, d, \mu] \in \mathbb{M}_{\mathrm{w}}$ for which $[X, d]$ is a geodesic metric space is closed as well, and a similar statement holds for $k$-pointed geodesic spaces, whose set is denoted by $\mathrm{PM}_{k}^{*}$.

Let $(X, d)$ be a compact geodesic metric space, and let $x, y \in X$ and $D \in(-d(x, y), d(x, y))$. A point $z \in X$ is called a $D$-median point between $x$ and $y$ if $d(x, z)-d(y, z)=D$. Note that the set $\operatorname{med}_{x y}^{D}(X)$ of such points is compact and separates the space $X$ into two open subsets, one containing $x$ and the other $y$, since every path $c:[0,1] \rightarrow X$ from $x$ to $y$ is such that $d(c(t), x)-d(c(t), y)$ ranges from $-d(x, y)$ to $d(x, y)$ continuously. We also say that $z$ is on a $\delta$-quasi geodesic from $x$ to $y$ if $d(x, z)+d(y, z) \leq d(x, y)+\delta$. We let $\mathrm{qg}_{x y}^{\delta}(X)$ be the set of such points, and medqg ${ }_{x y}^{D, \delta}(X)=\operatorname{med}_{x y}^{D}(X) \cap \operatorname{qg}_{x y}^{\delta}(X)$.

Proposition 11. - Let $\left[X_{n}, d_{n},\left(x_{n}, y_{n}\right)\right] \in \mathrm{PM}_{2}^{*}$ be converging to $[X, d,(x, y)]$, and let also $D_{n} \in\left(-d_{n}\left(x_{n}, y_{n}\right), d_{n}\left(x_{n}, y_{n}\right)\right)$ be converging to $D \in(-d(x, y), d(x, y))$. We assume that for every $\varepsilon>0$, we can find $\delta>0$ such that,

$$
\limsup _{n \rightarrow \infty} \operatorname{diam}\left(\operatorname{medqg}_{x_{n} y_{n}}^{D_{n}, \delta}\left(X_{n}\right)\right)<\varepsilon .
$$

Then there exists a unique element of $\operatorname{med}_{x y}^{D}(X)$ which is on a geodesic path from $x$ to $y$, i.e. $\operatorname{medqg}_{x y}^{D, 0}(X)$ is a singleton.

Proof. - Assume that there are two possibly different points $z, z^{\prime} \in \operatorname{med}_{x y}^{D}(X)$ that belong to some geodesic path between $x$ and $y$. Choose arbitrarily such a path $\gamma=\gamma_{x y}$ going through $z$ and let $\varepsilon>0$. Let $\varepsilon>\delta>0$ be as in the hypothesis for this value of $\varepsilon$, and let $0=t_{0}<t_{1}<\cdots<t_{K}=d(x, y)$ be such that $d\left(u^{k}, u^{k+1}\right) \leq \delta / 7$ where $u^{k}=\gamma\left(t_{k}\right)$. Let $\mathcal{R}_{n}$ be a correspondence between $X_{n}$ and $X$ such that $x_{n} \mathcal{R}_{n} x$ and $y_{n} \mathcal{R}_{n} y$, and dis $\mathcal{R}_{n} \rightarrow 0$ as $n \rightarrow \infty$. We may assume that $n$ is large enough so that dis $\mathcal{R}_{n} \leq \delta / 7$. Choose arbitrarily $u_{n}^{k} \mathcal{R}_{n} u^{k}$ and let $\gamma_{n}$ be the concatenation of arbitrarily chosen geodesic paths between $u_{n}^{k}$ and $u_{n}^{k+1}$ for $0 \leq k \leq K-1$.

Take a point $v$ on $\gamma_{n}$. Then for some $k, v$ lies on a geodesic from $u_{n}^{k}$ to $u_{n}^{k+1}$. Then

$$
\begin{aligned}
d_{n}\left(v, x_{n}\right) & \leq d_{n}\left(v, u_{n}^{k}\right)+d_{n}\left(u_{n}^{k}, x_{n}\right) \\
& \leq d_{n}\left(u_{n}^{k+1}, u_{n}^{k}\right)+d_{n}\left(u_{n}^{k}, x_{n}\right) \\
& \leq d\left(u^{k+1}, u^{k}\right)+d\left(u^{k}, x\right)+2 \operatorname{dis} \mathcal{R}_{n} \\
& \leq d\left(u^{k}, x\right)+2 \operatorname{dis} \mathcal{R}_{n}+\delta / 7 \leq d\left(u^{k}, x\right)+3 \delta / 7,
\end{aligned}
$$

so that by a symmetric inequality involving $y_{n}$ and by summing,

$$
d_{n}\left(v, x_{n}\right)+d_{n}\left(v, y_{n}\right) \leq d(x, y)+6 \delta / 7 \leq d_{n}\left(x_{n}, y_{n}\right)+\delta .
$$

Therefore the image of $\gamma_{n}$ is included in $\mathrm{qg}_{x_{n} y_{n}}^{\delta}\left(X_{n}\right)$. Let $z_{n}$ be any element of $\operatorname{med}_{x_{n} y_{n}}^{D_{n}}\left(X_{n}\right)$ that belongs to $\gamma_{n}$, and let $\tilde{z}_{n} \in X$ such that $z_{n} \mathcal{R}_{n} \tilde{z}_{n}$.

Now doing the same with a geodesic path $\gamma^{\prime}$ from $x$ to $y$ passing through $z^{\prime}$, we can construct elements $z_{n}^{\prime} \mathcal{R}_{n} \tilde{z}_{n}^{\prime}$ such that $z_{n}^{\prime} \in \operatorname{med}_{x_{n} y_{n}}^{D_{n}}\left(X_{n}\right) \cap \mathrm{qg}_{x_{n} y_{n}}^{\delta}\left(X_{n}\right)$. Hence $d_{n}\left(z_{n}, z_{n}^{\prime}\right) \leq \varepsilon$ for large $n$ by hypothesis, and thus $d\left(\tilde{z}_{n}, \tilde{z}_{n}^{\prime}\right) \leq \varepsilon+\operatorname{dis} \mathcal{R}_{n}$. 
Letting $n$ go to infinity, possibly through a subsequence, we obtain that $\tilde{z}^{n} \rightarrow \tilde{z}(\varepsilon)$ and $\tilde{z}_{n}^{\prime} \rightarrow \tilde{z}^{\prime}(\varepsilon)$, where necessarily $\tilde{z}(\varepsilon), \tilde{z}^{\prime}(\varepsilon) \in \operatorname{med}_{x y}^{D}(X)$ by passing to the limit in

$$
\left|d\left(\tilde{z}_{n}, x\right)-d\left(\tilde{z}_{n}, y\right)-D_{n}\right| \leq 2 \operatorname{dis} \mathcal{R}_{n}
$$

and similarly for $\tilde{z}_{n}^{\prime}$. By a similar argument, since $z_{n} \in \gamma_{n}$ we have $\tilde{z}(\varepsilon) \in \gamma^{\delta}$, the $\delta$-neighborhood of the image of $\gamma$. Since $\gamma^{\delta}$ decreases to $\gamma$ as $\delta \downarrow 0$ and $\operatorname{med}_{x y}^{D}(X)$ is compact, letting $\varepsilon$ (and $\delta$ ) decrease to $0, \tilde{z}(\varepsilon)$ converges to the unique element of $\gamma \cap \operatorname{med}_{x y}^{D}(X)$, which is $z$. Similarly, $\tilde{z}^{\prime}(\varepsilon)$ can be made as close to $z^{\prime}$ as wanted. But since $d\left(\tilde{z}_{n}, \tilde{z}_{n}^{\prime}\right) \leq \varepsilon+\operatorname{dis} \mathcal{R}_{n}$ we get that $d\left(\tilde{z}(\varepsilon), \tilde{z}^{\prime}(\varepsilon)\right) \leq \varepsilon$, and this entails $z=z^{\prime}$.

\section{Metric aspects of random quadrangulations}

We now embark in the proof of Theorems 2 and 3. We start with a preliminary remark. For technical reasons, the set PM and its weighted and marked analogs are somewhat easier to work with for our purposes. Of course, the main space of interest, namely $\mathrm{X}_{\mathbf{q}}$ for a quadrangulation $\mathbf{q}$, is not a geodesic metric space. However, its $\mathrm{d}_{\mathrm{GH}^{-}}$-distance to $\mathrm{PM}$ is at most 1. Indeed, take copies of the unit segment (with the usual metric) $\left\{x^{e}: 0 \leq x \leq 1\right\}$ indexed by an orientation $E_{1 / 2}(\mathbf{q})$ of the edges of $\mathbf{q}$, and identify the points $0^{e}$ for which $e^{-}$coincide, and the points $1^{e}$ for which $e^{+}$coincide. The quotient metric graph [9, Chapter 3.2.2] is a geodesic metric space whose restriction to the points $0^{e}, e \in E_{1 / 2}(\mathbf{q})$ is isometric to $\left(V(\mathbf{q}), d_{\mathbf{q}}\right)$, and obviously its Gromov-Hausdorff distance to the latter is less than 1 . Thus any limit in distribution of $a^{-1 / 4} \mathrm{X}_{\mathbf{q}}$ must be at 0 distance from PM with probability 1 , hence belong to PM. In passing this proves property 1 in Theorem 3. For these reasons, we work with the metric graph constructed above, which we still call $\mathrm{X}_{\mathbf{q}}$, and restrict our attention to geodesic metric spaces from now on.

\subsection{Proof of Theorem 2}

For $\mathrm{X} \in \mathbb{M}$ and $\varepsilon>0$, we let $\mathcal{N}(\mathrm{X}, \varepsilon)$ be the minimal number of open balls of radius $\leq \varepsilon$ that are needed to cover $\mathrm{X}$. For any sequence $N=\left(N_{k}, k \geq 1\right)$ of positive integers, we introduce the set

$$
\mathbb{K}_{N}=\left\{\mathrm{X}^{\mathrm{w}}=[X, d, \mu] \in \mathrm{PM}_{\mathrm{w}}: \mathcal{N}\left([X, d], 2^{-k}\right) \leq N_{k} \forall k \geq 1\right\} .
$$

By Propositions 8 and 5 and [9, Exercise 7.4.14], the sets $\mathbb{K}_{N}$ are (relatively) compact. By Prokhorov's theorem, it is thus sufficient to show that for every $\varepsilon>0$, there is a choice of $N, \Delta$ for which

$$
\mathrm{Q}_{g}^{(\beta / a)}\left(\left\{a^{-1 / 4} \mathrm{X}_{\mathbf{q}}^{\mathbf{w}} \in \mathbb{K}_{N}, \Delta^{-1} \leq a^{-1} V_{\mathbf{q}} \leq \Delta\right\}\right) \geq 1-\varepsilon .
$$

To do so, we use the results of Sections $2-4$ in the case $k=1$. We also separate the case $g \geq 1$ and $g=0$, starting with the former. Consider $(\mathbf{q}, x) \in \mathbf{Q}_{g, 1}$ and let $(\mathbf{m},[\mathbf{l}])=\Psi_{g, 1}(\mathbf{q}, x)$ and $\left(\mathfrak{m}, \mathfrak{l}, t^{*}\right)=\widetilde{\Psi}_{g, 1}(\mathbf{q}, x)$. Recall also the definition (11) of $l$, and write

$$
\omega(h, \varepsilon)=\sup _{x, y \in J,|x-y| \leq \varepsilon}|h(x)-h(y)|
$$

for the modulus of continuity of a function $h$ defined on an interval $J$.

$4{ }^{\mathrm{e}}$ SÉRIE - TOME $42-2009-\mathrm{N}^{\circ} 5$ 
Lemma 16. - For $R>4$,

$$
\left\{\mathcal{N}\left(\mathrm{X}_{\mathbf{q}}, R\right)>N+1\right\} \subset\left\{2 \omega\left(l,\left\lceil\frac{\tau_{\mathfrak{l}}}{N}\right\rceil\right) \geq R / 2\right\} .
$$

Proof. - Since $\mathbf{m}$ has only one face of degree $\tau_{\mathfrak{l}}$, all the half-edges of $\mathbf{m}$ are of the form $e(j)=\varphi_{\mathbf{m}}^{j}\left(\mathfrak{e}_{*}(0)\right)$ for some integer $j \in\left[0, \tau_{\mathfrak{l}}\right)$ with the notations of Section 3.3. Split the lifetime interval $\left[0, \tau_{\mathfrak{l}}\right]$ of $l$ into $N$ disjoint parts $I_{1}, \ldots, I_{N}$ of lengths at most $\left\lceil\tau_{\mathfrak{l}} / N\right\rceil$. By Lemma 3 and the definition (10) of $l$, if $\omega\left(l,\left\lceil\tau_{\mathfrak{l}} / N\right\rceil\right)<R / 2-1$, then all the vertices of $\mathbf{m}$ of the form $e(j)^{-}$for $j \in I_{i}$, and considered as vertices of $\mathbf{q}$, must be within $d_{\mathbf{q}}$-distance at most $R$, for each $1 \leq i \leq N$. Since all vertices of $\mathbf{q}$ are contained in this way except the distinguished vertex $x$, adding yet another ball to cover $x$ yields (25).

We now consider a marked version of $\mathbf{Q}_{g}^{(\beta)}$, as the probability on $\mathbf{Q}_{g, 1}$ defined by

$$
\mathrm{Q}_{g}^{(\beta)}(\mathrm{d} \mathbf{q}) \mu_{\mathbf{q}}(\mathrm{d} x)=\frac{\mathrm{Q}_{g, 1}\left(V_{\mathbf{q}}^{-1} e^{-\beta V_{\mathbf{q}}} \mathrm{d}(\mathbf{q}, x)\right)}{\mathbf{Q}_{g, 1}\left(V_{\mathbf{q}}^{-1} e^{-\beta V_{\mathbf{q}}}\right)} .
$$

Using Section 4 , its image under $\widetilde{\Psi}_{g, 1}$ can be written

$$
\operatorname{LM}_{g, 1}^{(\beta)}:=\frac{\operatorname{LM}_{g, 1}\left(\left(\tau_{\mathfrak{l}} / 2+\chi(g)\right)^{-1} \exp \left(-\beta \tau_{\mathfrak{l}} / 2\right) \mathrm{d}\left(\mathfrak{m}, \mathfrak{l}, t^{*}\right)\right)}{\operatorname{LM}_{g, 1}\left(\left(\tau_{\mathfrak{l}} / 2+\chi(g)\right)^{-1} \exp \left(-\beta \tau_{\mathfrak{l}} / 2\right)\right)}
$$

Now, Theorem 5 (and elementary bounds to get rid of the term $\chi(g)$ ) entail that the law of $\xi^{a}\left(\mathfrak{m}, \mathfrak{l}, t^{*}\right)$ under $\mathrm{LM}_{g, 1}^{(\beta / a)}$ converges weakly towards

$$
\operatorname{CLM}_{g, 1}^{(\beta)}:=\frac{\operatorname{CLM}_{g, 1}\left(\tau_{\mathfrak{l}}^{-1} \exp \left(-\beta \tau_{\mathfrak{l}}\right) \mathrm{d}\left(\mathfrak{m}, \mathfrak{l}, t^{*}\right)\right)}{\operatorname{CLM}_{g, 1}\left(\tau_{\mathfrak{l}}^{-1} \exp \left(-\beta \tau_{\mathfrak{l}}\right)\right)} .
$$

By the continuous mapping theorem, the distribution of $\left(\psi^{a}\left(l^{\mathfrak{e}}\right), \mathfrak{e} \in E(\mathfrak{m})\right)$ under $\operatorname{LM}_{g, 1}^{(\beta / a)}$ jointly converges towards the law of the processes $\left(l^{\mathfrak{e}}, \mathfrak{e} \in E(\mathfrak{m})\right)(12)$ under $\operatorname{CLM}_{g, 1}^{(\beta)}$, and thus the law of $\psi^{a}(l)$ under $\operatorname{LM}_{g, 1}^{(\beta / a)}$ converges towards the law of $l(11)$ under $\operatorname{CLM}_{g, 1}^{(\beta)}$. Combined with the fact from Lemma 16 that for $a$ large enough,

$$
\left\{\mathcal{N}\left(a^{-1 / 4} \mathrm{X}_{\mathbf{q}}, \delta\right)>N+1\right\} \subset\left\{2 \omega\left(\psi^{a}(l),(2 a)^{-1}\left\lceil\frac{\tau_{\mathfrak{l}}}{N}\right\rceil\right) \geq \delta / 2\right\}
$$

and by usual relative compactness criteria derived from the Ascoli-Arzela theorem [5, Theorem 8.2], we see that for every $k \geq 1$, there exists $N_{k}$ such that for every $a$, $\mathrm{Q}_{g}^{(\beta / a)}\left(\mathcal{N}\left(a^{-1 / 4} \mathrm{X}_{\mathbf{q}}, 2^{-k}\right)>N_{k}\right) \leq \varepsilon 2^{-k}$. This entails $\mathrm{Q}_{g}^{(\beta / a)}\left(a^{-1 / 4} \mathrm{X}_{\mathbf{q}} \in \mathbb{K}_{N}\right) \geq 1-\varepsilon$ for this choice of $N=\left(N_{k}, k \geq 1\right)$. We see as well that the random variables $V_{\mathbf{q}} / a$ are tight in $(0, \infty)$ as they converge in distribution towards the law of $\tau_{\mathfrak{l}}$ under $\operatorname{CLM}_{g, 1}^{(\beta)}$.

The case $g=0$ is similar but simpler, the role of $\mathrm{CLM}_{0,1}$ being performed by the Itô measure of the Brownian snake, and taking into account the factor $V_{\mathbf{q}}^{2}$ appearing in the definition of $\mathrm{Q}_{0}^{(\beta)}$, we must replace the limit in law of $\psi^{a}(l)$ by the law of $z$ under

$$
\frac{\mathrm{CLM}_{0,1}(\tau \exp (-\beta \tau) \mathrm{d}(c, z))}{\mathrm{CLM}_{0,1}(\tau \exp (-\beta \tau))} .
$$

One will notice that the denominator would diverge if it were not for the corrective term $\tau$ in front of the exponential, since $\operatorname{CLM}_{0,1}(\tau \in \mathrm{d} t)=4\left(2 \pi t^{3}\right)^{-1 / 2} \mathrm{~d} t$. The statement of Lemma 16 remains true without change, and we conclude in the same way. 


\subsection{Proof of Theorem 3}

Property 1 of this theorem has been discussed at the beginning of this section. Let us set things up for Property 2. We give the proofs for $g \geq 1$, the case $g=0$ being similar and easier.

7.2.1. Diffuseness of $\mu$. - By Theorem 2, there exists an increasing sequence $\left(a_{n}, n \geq 1\right)$ along which the distribution under $\mathrm{Q}_{g}^{(\beta / a)}$ of $\left(a^{-1 / 4} \mathrm{X}_{\mathbf{q}}^{\mathrm{w}}, a^{-1} V_{\mathbf{q}}\right)$ converges to a limit $\mathscr{S}_{g}^{(\beta)}$. From now on, we will always suppose that $a$ is taken along this sequence. From this and the discussion above (26), we obtain that the laws of $\left(a^{-1 / 4} \mathrm{X}_{\mathbf{q}}^{\mathrm{w}}, a^{-1} V_{\mathbf{q}}, \xi^{a}\left(\widetilde{\Psi}_{g, 1}(\mathbf{q}, x)\right)\right)$ under $\mathbf{Q}_{g}^{(\beta / a)}(\mathrm{d} \mathbf{q}) \mu_{\mathbf{q}}(\mathrm{d} x)$ form a relatively compact family of probability distributions on $\mathrm{PM}_{\mathrm{w}} \times(0, \infty) \times \mathbf{C}_{g, 1}$. Up to a further extraction, and by a use of Skorokhod's embedding theorem, we may and will assume that we are working on a probability space $(\Omega, \mathcal{F}, P)$ under which are defined random variables $\left(\mathbf{q}_{a}, x_{a}\right)$ with respective laws $\mathrm{Q}_{g}^{(\beta / a)}(\mathrm{d} \mathbf{q}) \mu_{\mathbf{q}}(\mathrm{d} x)$ such that

1. letting $\mathrm{X}_{a}^{\mathrm{w}}=\left[X_{a}, d_{a}, \mu_{a}\right]:=\mathrm{X}_{\mathbf{q}_{a}}^{\mathrm{w}}$, we have $a^{-1 / 4} \mathrm{X}_{a}^{\mathrm{w}} \rightarrow \mathrm{X}^{\mathrm{w}}=[X, d, \mu]$, a random variable with values in $\mathrm{PM}_{\mathrm{w}}$ (and we let $\mathrm{X}_{a}=\left[X_{a}, d_{a}\right]$ ),

2. letting $\quad\left(\mathbf{m}_{a},\left[\mathbf{l}_{a}\right]\right)=\Psi_{g, 1}\left(\mathbf{q}_{a}, x_{a}\right) \quad$ and $\quad\left(\mathfrak{m}_{a}, \mathfrak{l}_{a}, t_{a}^{*}\right)=\widetilde{\Psi}_{g, 1}\left(\mathbf{q}_{a}, x_{a}\right)$, then $\xi^{a}\left(\mathfrak{m}_{a}, \mathfrak{l}_{a}, t_{a}^{*}\right) \rightarrow\left(\mathfrak{m}, \mathfrak{l}, t^{*}\right)$, a random variable with law $\operatorname{CLM}_{g, 1}^{(\beta)}$,

these convergences holding almost-surely as $a \rightarrow \infty$ (along the proper sequence). We will adopt the notation $\mathfrak{l}_{a}=\left(w_{a}^{\mathfrak{e}}, c_{a}^{\mathfrak{e}}, z_{a}^{\mathfrak{e}}, \mathfrak{e} \in E\left(\mathfrak{m}_{a}\right)\right), l_{a}^{\mathfrak{e}}=z_{a}^{\mathfrak{e}}+w_{a}^{\mathfrak{e}} \circ \underline{c}_{a}^{\mathfrak{e}}$, let $\tau_{a}^{\mathfrak{e}}$ be the duration of $c_{a}^{\mathfrak{e}}$, and let $l_{a}$ be the process defined as in (11) or (10). Note that $(2 a)^{-1} \tau_{\mathfrak{l}_{a}}$ and $a^{-1} V_{\mathbf{q}_{a}}$ both converge to $\tau_{\mathfrak{l}}$ as $a \rightarrow \infty$, so the random variable $\left(\mathrm{X}^{\mathrm{w}}, \tau_{\mathfrak{l}}\right)$ has law $\mathscr{S}_{g}^{(\beta)}$. By the previous convergences, the processes $\psi^{a}\left(l_{a}^{\mathfrak{e}}\right)$ converge to $l^{\mathfrak{e}}$, so that $\psi^{a}\left(l_{a}\right)$ converges a.s. as $a \rightarrow \infty$ towards the concatenated process $l$ associated with $\left(\mathfrak{m}, \mathfrak{l}, t^{*}\right)$.

Lemma 17. - We have

$$
\begin{aligned}
\int_{a^{-1 / 4} X_{a}} & \mu_{a}(\mathrm{~d} x) \mu_{a}(B(x, \varepsilon)) \\
\leq & \left(\frac{4 a}{\tau_{\mathfrak{l}_{a}}}\right)^{2} \int_{\left[0, \tau_{\mathfrak{I}_{a}} /(2 a)\right)^{2}} \mathrm{~d} u \mathrm{~d} v \mathbb{1}_{\left\{\left|\psi^{a}\left(l_{a}\right)(\lfloor 2 a u\rfloor /(2 a))-\psi^{a}\left(l_{a}\right)(\lfloor 2 a v\rfloor /(2 a))\right|<\varepsilon / \varsigma\right\}}+4 \tau_{\mathfrak{l}_{a}}^{-1} .
\end{aligned}
$$

Proof. - With each integer time $i$ corresponds the exploration of a corner $e(i)=\varphi_{\mathbf{m}_{a}}^{i}\left(\mathfrak{e}_{*}(0)\right)$ of $\mathbf{m}_{a}$, and each corner of $\mathbf{m}_{a}$ is explored in this way because $\mathbf{m}_{a}$ has only one face. Considering the vertices $e(i)^{-}$as vertices of $\mathbf{q}_{a}$, we have $d_{a}\left(e(i)^{-}, e(j)^{-}\right) \geq\left|l_{a}(i)-l_{a}(j)\right|$ by Lemma 3. From this, it follows that

$$
\sum_{x, y \in V\left(\mathbf{q}_{a}\right)} \mathbb{1}_{\left\{d_{a}(x, y)<a^{1 / 4} \varepsilon\right\}} \leq \int_{\left[0, \tau_{\mathfrak{\tau}_{a}}\right)^{2}} \mathrm{~d} u \mathrm{~d} v \mathbb{1}_{\left\{\left|l_{a}(\lfloor u\rfloor)-l_{a}(\lfloor v\rfloor)\right|<a^{1 / 4} \varepsilon\right\}}+2\left(\tau_{\mathfrak{l}_{a}} / 2+\chi(g)\right),
$$

where the last term amounts from the fact that the selected vertex $x_{a}$ is the only vertex of $\mathbf{q}_{a}$ not corresponding to a vertex of $\mathbf{m}_{a}$, and a rough upper bound. We conclude by dividing by $\left(\tau_{\mathfrak{r}_{a}} / 2+\chi(g)\right)^{2}=V_{\mathbf{q}_{a}}^{2}$ and a linear change of variables.

Lemma 18. - Almost-surely, the level sets $\left\{t \in\left[0, \tau_{\mathfrak{l}}\right): l(t)=x\right\}$ have zero Lebesgue measure for every $x \in \mathbb{R}$. 
Proof. - From the construction of $l$ as the concatenation of the paths $l^{\mathfrak{e}}$, and given $\mathfrak{m}$, it suffices to show the same property for the processes $l^{\mathfrak{e}}=z^{\mathfrak{e}}+w^{\mathfrak{e}} \circ \underline{c}^{\mathfrak{e}}$ of (12). Under $\mathrm{CLM}_{g, 1}$, recall that conditionally on $\left(w^{\mathfrak{e}}, c^{\mathfrak{e}}, \mathfrak{e} \in E(\mathfrak{m})\right),\left(z^{\mathfrak{e}}(t), 0 \leq t \leq \tau^{\mathfrak{e}}\right)$ is a Gaussian process with covariance $\operatorname{Cov}\left(z^{\mathfrak{e}}(s), z^{\mathfrak{e}}(t)\right)=\inf _{s \wedge t \leq u \leq s \vee t} c^{\mathfrak{e}}(u)$, by definitions of snakes. Therefore, under this conditioned measure, the laws of pairs $\left(l^{\mathfrak{l}}(s), l^{\mathfrak{e}}(t)\right)$ have densities, and consequently, for fixed $s, t$,

$$
\operatorname{CLM}_{g, 1}\left(\left\{l^{\mathfrak{e}}(s)=l^{\mathfrak{e}}(t), \tau^{\mathfrak{e}} \geq s \vee t\right\}\right)=0,
$$

and the same is true for $\operatorname{CLM}_{g, 1}^{(\beta)}$ instead of $\operatorname{CLM}_{g, 1}$. By Fubini's theorem, and with a slight abuse of notation, this implies that for any $T>0$, if $U, V$ are uniform, independent random variables on $[0, T]$ independent of $\left(\mathfrak{m}, \mathfrak{l}, t_{*}\right)$ then it holds that

$$
\operatorname{CLM}_{g, 1}^{(\beta)}\left(\left\{l^{\mathfrak{e}}(U)=l^{\mathfrak{e}}(V), \tau^{\mathfrak{e}} \geq T\right\}\right)=0 .
$$

But obviously, if some level set $\left\{l^{e}=x\right\}$ had positive Lebesgue measure with positive probability, then the latter probability would be positive for some $T>0$.

We now conclude that $\mu$ is diffuse. Indeed, by the convergence of $\psi^{a}\left(l_{a}\right)$ to $l$ and of $\tau_{\mathfrak{l}_{a}} /(2 a)$ to $\tau_{\mathfrak{l}} \in(0, \infty)$, we obtain from Lemma 17 and dominated convergence that

$$
\liminf _{a \rightarrow \infty} \int_{a^{-1 / 4} X_{a}} \mu_{a}(\mathrm{~d} x) \mu_{a}(B(x, \varepsilon)) \leq \frac{16}{\tau_{\mathfrak{l}}^{2}} \int_{\left[0, \tau_{\mathfrak{l}}\right)^{2}} \mathrm{~d} u \mathrm{~d} v \mathbb{1}_{\{|l(u)-l(v)| \leq \varepsilon\}} .
$$

The last integral decreases to $\int_{\left[0, \tau_{\mathfrak{l}}\right)^{2}} \mathrm{~d} u \mathrm{~d} v \mathbb{1}_{\{l(u)=l(v)\}}$ as $\varepsilon \rightarrow 0$, and this is 0 by Lemma 18 . Lemma 14 allows us to conclude.

7.2.2. Support of $\mu$. - The setting is the same as in the previous section. For $e \in E\left(\mathbf{m}_{a}\right)$ we let $x(e)=e^{+}$if $d_{\mathbf{m}_{a}}\left(e^{+}, V\left(\mathbf{m}_{a}^{\geq 2}\right)\right)>d_{\mathbf{m}_{a}}\left(e^{-}, V\left(\mathbf{m}_{a}^{\geq 2}\right)\right)$ and $x(e)=e^{-}$otherwise (intuitively, it selects the vertex incident to $e$ that is the furthest away from $\mathbf{m}^{\geq 2}$ ). For $u \in\left[0, \tau_{\mathfrak{r}_{a}}\right)$ we let

$$
\langle u\rangle_{a}=\left\{\begin{array}{l}
\lfloor u\rfloor \text { if } x(e(\lfloor u\rfloor))=e(\lfloor u\rfloor)^{-} \\
\lceil u\rceil \text { if } x(e(\lfloor u\rfloor))=e(\lfloor u\rfloor)^{+}
\end{array}\right.
$$

Lemma 19. - The image measure of $2^{-1} \mathrm{~d} u \mathbb{1}_{\left[0, \tau_{\tau_{a}}\right)}(u)$ by $u \mapsto e\left(\langle u\rangle_{a}\right)^{-}$is the measure on $V\left(\mathbf{m}_{a}\right)=V\left(\mathbf{q}_{a}\right) \backslash\left\{x_{a}\right\}$ assigning mass 1 on every vertex of $V\left(\mathbf{m}_{a}\right) \backslash V\left(\mathfrak{m}_{a}\right)$, where $\mathfrak{m}_{a}$ is seen as a subgraph of $\mathbf{m}_{a}$, and assigning mass $\operatorname{deg}_{\mathfrak{m}_{a}}(x) / 2$ to every vertex $x \in V\left(\mathfrak{m}_{a}\right)$, the latter denoting the degree in the graph $\mathfrak{m}_{a}$.

Proof. - Assume $x$ is an element of $V\left(\mathbf{m}_{a}\right) \backslash V\left(\mathbf{m}_{a}^{\geq 2}\right)$. Then $e\left(\langle u\rangle_{a}\right)^{-}=x$ if and only if $i=\lfloor u\rfloor$ is either the first integer such that $e(i)^{+}=x$ or the last integer such that $e(i)^{-}=x$, corresponding to the two visits of the parent edge of $x$ in the tree rooted on $V\left(\mathbf{m}^{\geq 2}\right)$ containing $x$. The total mass of such $u$ 's is thus $1 / 2+1 / 2=1$.

Next, assume that $x \in V\left(\mathbf{m}_{a}^{\geq 2}\right) \backslash V\left(\mathfrak{m}_{a}\right)$. This time $e\left(\langle u\rangle_{a}\right)^{-}=x$ if $\lfloor u\rfloor$ is one of the two integers $i$ such that $e(i) \in E\left(\mathbf{m}_{a}^{\geq 2}\right)$ and $e(i)^{-}=x$.

Finally, if $x \in V\left(\mathfrak{m}_{a}\right)$ then $e\left(\langle u\rangle_{a}\right)^{-}=x$ if $\lfloor u\rfloor$ is an integer $i$ such that $e(i) \in E\left(\mathbf{m}_{\bar{a}}^{\geq 2}\right)$ and $e(i)^{-}=x$. There are $\operatorname{deg}_{\mathfrak{m}_{a}}(x)$ such integers. 
The measure appearing in Lemma 19 is of total mass $\tau_{\mathfrak{r}_{a}} / 2$, and we let $\tilde{\mu}_{a}$ be the probability measure obtained by renormalizing it by this quantity. Obviously, the Prokhorov distance in $a^{-1 / 4} \mathrm{X}_{a}$ between $\mu_{a}$ and $\tilde{\mu}_{a}$ vanishes as $a \rightarrow \infty$, so that $\left[X_{a}, a^{-1 / 4} d_{a}, \tilde{\mu}_{a}\right]$ converges to the same limit $[X, d, \mu]$ as $\left[X_{a}, a^{-1 / 4} d_{a}, \mu_{a}\right]$.

Lemma 20. - It holds that for every $\varepsilon>0$ and a large enough

$$
\begin{aligned}
\min _{x \in V\left(\mathbf{q}_{a}\right) \backslash\left\{x_{a}\right\}} \tilde{\mu}_{a}\left(B_{a^{-1 / 4} X_{a}}(x, \varepsilon)\right) \\
\quad \geq \min _{s \in\left[0, \tau_{\tau_{a}} /(2 a)\right)} \frac{2 a}{\tau_{\mathfrak{l}_{a}}} \int_{0}^{\tau_{\mathfrak{l}_{a}} /(2 a)} \mathrm{d} u \mathbb{1}_{\left\{\omega\left(\psi^{a}\left(l_{a}\right),\left|\langle 2 a u\rangle_{a}-2 a s\right| /(2 a)\right) \leq \varepsilon / 2\right\}} .
\end{aligned}
$$

Proof. - Let $x$ be a vertex in $V\left(\mathbf{q}_{a}\right) \backslash\left\{x_{a}\right\}$, and let $i_{x}$ be an integer such that $e\left(i_{x}\right)^{-}=x$. If $j$ is another integer time, then

$$
d_{a}\left(x, e(j)^{-}\right) \leq l_{a}(j)+l_{a}\left(i_{x}\right)-2 \min _{j \wedge i_{x} \leq u \leq j \vee i_{x}} l_{a}(u)+2 \leq 2 \omega\left(l_{a},\left|j-i_{x}\right|\right)+2
$$

by Lemma 3. Thus

$$
\begin{aligned}
\left(\tau_{\iota_{a}} / 2\right) \tilde{\mu}_{a}\left(\left\{y \in V\left(\mathbf{q}_{a}\right): d_{a}(x, y)<a^{1 / 4} \varepsilon\right\}\right) & =\frac{1}{2} \int_{0}^{2 \tau_{\digamma_{a}}} \mathrm{~d} u \mathbb{1}\left\{d_{a}\left(x, e\left(\langle u\rangle_{a}\right)^{-}\right)<a^{1 / 4} \varepsilon\right\} \\
& \geq \frac{1}{2} \int_{0}^{2 \tau_{\tau_{a}}} \mathrm{~d} u \mathbb{1}\left\{\omega\left(l_{a},\left|\langle u\rangle_{a}-i_{x}\right|\right)<a^{1 / 4} \varepsilon / 2-1\right\},
\end{aligned}
$$

where we used Lemma 19 in the first step. The result is obtained by taking the infimum over $x$ and by simple rearrangements.

Since $\psi^{a}\left(l_{a}\right)$ converges uniformly to the continuous limit $l$, there exists $\eta$ such that for every (large enough) $a, \omega\left(\psi^{a}\left(l_{a}\right), \eta\right) \leq \varepsilon / 2$. From this, we readily obtain from the last lemma that

$$
\limsup _{a \rightarrow \infty} \inf _{x \in V\left(\mathbf{q}_{a}\right) \backslash\left\{x_{a}\right\}} \tilde{\mu}_{a}\left(B_{a^{-1 / 4} X_{a}}(x, \varepsilon)\right) \geq \eta / 2 \tau_{\mathfrak{l}}>0 .
$$

Since $V\left(\mathbf{q}_{a}\right) \backslash\left\{x_{a}\right\}$ is $a^{-1 / 4}$-dense in $\mathrm{X}_{a}$, one concludes by Lemma 15 that $\mu$ is of full support.

7.2.3. Uniqueness of geodesics. - We now specialize the results of Section 4 to the case $k=2$, starting with some general remarks. A delay $D=\left[d_{1}, d_{2}\right] \in \mathcal{D}\left(\mathbf{q},\left(x_{1}, x_{2}\right)\right)$ is identified with the quantity $d_{1}-d_{2}$, which is an integer in the interval $\left(-d_{\mathbf{q}}\left(x_{1}, x_{2}\right), d_{\mathbf{q}}\left(x_{1}, x_{2}\right)\right)$ such that $D+d_{\mathbf{q}}\left(x_{1}, x_{2}\right)$ is even.

Let $\left(\mathbf{q},\left(x_{1}, x_{2}\right), D\right) \in \mathbf{Q}_{g, 2}$ and $(\mathbf{m},[\mathbf{l}])=\Psi_{g, 2}\left(\mathbf{q},\left(x_{1}, x_{2}\right), D\right)$. Consider any chain in $\mathbf{q}$ from $x_{1}$ to $x_{2}$ with length $d_{\mathbf{q}}\left(x_{1}, x_{2}\right)$. There must be a (unique) vertex $x$ in this chain that belongs to $V_{1} \cap V_{2}$ (where $V_{i}=V_{i}(\mathbf{q}, \mathbf{x}, D)$ ), i.e. for which $d_{\mathbf{q}}\left(x_{1}, x\right)-d_{\mathbf{q}}\left(x_{2}, x\right)=D$. We claim that $\mathbf{l}(x)=\min _{V_{1} \cap V_{2}} \mathbf{l}(y)$. Indeed, we know from Theorem 4 that $d_{\mathbf{q}}\left(y, x_{1}\right)=\mathbf{l}(y)-\mathbf{l}\left(x_{1}\right)$ and $d_{\mathbf{q}}\left(y, x_{2}\right)=\mathbf{l}(y)-\mathbf{l}\left(x_{2}\right)$ for $y \in V_{1} \cap V_{2}$, and since $d_{\mathbf{q}}\left(x, x_{1}\right)+d_{\mathbf{q}}\left(x, x_{2}\right)=d_{\mathbf{q}}\left(x_{1}, x_{2}\right)$ since $x$ is on a geodesic, there must be equality in the triangle inequality 
$2 \mathbf{l}(y)-\mathbf{l}\left(x_{1}\right)-\mathbf{l}\left(x_{2}\right) \geq d_{\mathbf{q}}\left(x_{1}, x_{2}\right)$, and $\mathbf{l}(x)$ is indeed minimal. In passing, we have obtained the formula

$$
\begin{aligned}
d_{\mathbf{q}}\left(x_{1}, x_{2}\right) & =2 \min _{e \in f_{1}: \bar{e} \in f_{2}} \mathbf{l}(e)-\mathbf{l}\left(x_{1}\right)-\mathbf{l}\left(x_{2}\right) \\
& =2 \min _{e \in f_{1}: \bar{e} \in f_{2}} \mathbf{l}(e)-\min _{e \in f_{1}} \mathbf{l}(e)-\min _{e \in f_{2}} \mathbf{l}(e)-2 \\
& =2 \min _{e \in f_{*}: \bar{e} \in \bar{f}_{*}} \mathbf{l}(e)-\min _{e \in f_{*}} \mathbf{l}(e)-\min _{e \in \bar{f}_{*}} \mathbf{l}(e)-2,
\end{aligned}
$$

where $f_{*}$ is the face of $\mathbf{m}$ incident to its root $e_{*}$, and $\bar{f}_{*}$ is the other face. Indeed, with a vertex in $V_{1} \cap V_{2}$ corresponds a vertex of $\mathbf{m}$ incident to both $f_{1}$ and $f_{2}$, and such a vertex is the origin of at least one edge incident to $f_{1}$ with reversal incident to $f_{2}$. This gives the first equality, and the second one follows by symmetry in the indices of the second formula.

For an element $\left(\mathfrak{m}, \mathfrak{l}, t^{*}\right) \in \mathbf{C}_{g, 2}$, we let $\mathfrak{f}_{*}$ be the face of $\mathfrak{m}$ incident to $\mathfrak{e}_{*}$, and $\overline{\mathfrak{f}}_{*}$ be the other face. Let

$$
\mathfrak{e}_{i}:=\varphi_{\mathfrak{m}}^{i-1}\left(\mathfrak{e}_{*}\right), \quad 1 \leq i \leq \operatorname{deg}_{\mathfrak{m}}\left(\mathfrak{f}_{*}\right)
$$

be the list of edges of $\mathfrak{m}$ incident to $\mathfrak{f}_{*}$ (this notation should not be confused with the notation $\mathfrak{e}(i)$, which denotes edges of $\mathbf{m})$. Besides the process $l$ defined at (10) we will need two other auxiliary processes. First, let $i_{0}$ be the first index $i$ such that $\overline{\mathfrak{e}}_{i} \in \overline{\mathfrak{f}}_{*}$. Let

$$
\mathfrak{e}_{1}^{\prime}=\varphi_{\mathfrak{m}}\left(\overline{\mathfrak{e}}_{i_{0}}\right)=\sigma_{\mathfrak{m}}^{-1}\left(\varphi_{\mathfrak{m}}^{i_{0}-1}\left(\mathfrak{e}_{*}\right)\right),
$$

which is incident to $\overline{\mathfrak{f}}_{*}$ and has the same origin as $\mathfrak{e}_{i_{0}}$, and

$$
\mathfrak{e}_{i}^{\prime}=\varphi_{\mathfrak{m}}^{i-1}\left(\mathfrak{e}_{1}^{\prime}\right), \quad 1 \leq i \leq \operatorname{deg}_{\mathfrak{m}}\left(\overline{\mathfrak{f}}_{*}\right) .
$$

We define a process $l^{\prime}$ as the concatenation

$$
l^{\prime}=l^{\mathfrak{e}_{1}^{\prime}} \ldots l^{\mathfrak{e}^{\prime} \operatorname{deg}_{\mathfrak{m}}\left(\overline{\mathfrak{f}}_{*}\right)} .
$$

Next, let $w$ be defined as the concatenation

$$
w=w^{\mathfrak{e}_{*}} w^{\varphi_{\mathfrak{m}}\left(\mathfrak{e}_{*}\right)} \ldots w^{\varphi_{\mathfrak{m}}^{\operatorname{deg}_{\mathfrak{m}}\left(\mathfrak{e}_{*}\right)-1}\left(\mathfrak{e}_{*}\right)} .
$$

We also consider the function defined for $0 \leq t \leq \tau(w)$ by $\Upsilon(t)=i$ if $\sum_{0 \leq j<i} r_{\varphi_{\mathfrak{m}}^{j}\left(\mathfrak{e}_{*}\right)} \leq t<\sum_{0 \leq j \leq i} r_{\varphi_{\mathfrak{m}}^{j}\left(\mathfrak{e}_{*}\right)}$, and $\Upsilon(\tau(w))=\operatorname{deg}_{\mathfrak{m}}\left(\mathfrak{f}_{*}\right)-1$.

When $(\mathbf{m},[\mathbf{l}])=\Psi_{g, 2}(\mathbf{q}, \mathbf{x}, D)$ and $\left(\mathfrak{m}, \mathfrak{l}, t^{*}\right)=\Xi_{g, 2}(\mathbf{m},[\mathbf{l}])$ for some $(\mathbf{q}, \mathbf{x}, D) \in \mathbf{Q}_{g, 2}$, it is straightforward, though a little tedious to check formally, that $l^{\prime}$ is alternatively described as the process interpolating linearly between the values

$$
l^{\prime}(i)=\mathbf{l}\left(\varphi_{\mathbf{m}}^{i}\left(e_{1}^{\prime}\right)\right)-\mathbf{l}\left(e_{1}^{\prime}\right), \quad 0 \leq i \leq \operatorname{deg}_{\mathbf{m}}\left(\bar{f}_{*}\right)-1,
$$

and $l^{\prime}\left(\operatorname{deg}_{\mathbf{m}}\left(\bar{f}_{*}\right)\right)=0$, where

$$
e_{1}^{\prime}=\sigma_{\mathbf{m}}^{-1} \varphi_{\mathbf{m}}^{j_{0}}\left(e_{*}\right),
$$

and where $j_{0}$ is the first index $j$ such that $\overline{\varphi_{\mathbf{m}}^{j_{0}}\left(e_{*}\right)} \in \bar{f}_{*}$. One checks in this case that

$$
\mathbf{l}\left(e_{1}^{\prime}\right)=\sum_{i=1}^{i_{0}-1} \widehat{l}^{\mathfrak{e}_{i}},
$$

so that the function $\sum_{i=1}^{i_{0}-1} \widehat{l}^{\mathfrak{e}_{i}}+l^{\prime}$ reproduces faithfully the labels of half-edges incident to $\bar{f}_{*}$. Still in this case, the function $w$ reproduces the labels $\mathbf{l}$ along the face of $\mathbf{m}^{\geq 2}$ incident 
to $e_{*}$, while $\Upsilon(t)$ records the label of the edge of $\mathfrak{m}$ on which the edge of $\mathbf{m}^{\geq 2}$ explored at time $\lfloor t\rfloor$ projects.

Lemma 21. - Let $(\mathbf{q}, \mathbf{x}, D) \in \mathbf{Q}_{g, 2}$ and $\left(\mathfrak{m}, \mathfrak{l}, t^{*}\right)=\widetilde{\Psi}_{g, 2}(\mathbf{q}, \mathbf{x}, D)$. Assume that $\min \left\{w(t): \overline{\mathfrak{e}}_{\Upsilon(t)} \in \overline{\mathfrak{f}}_{*}\right\}$ is attained for a unique value $i_{m}$ of $\Upsilon_{t}$, such that $\overline{\mathfrak{e}}_{i_{m}} \in \overline{\mathfrak{f}}_{*}:$ Also assume that for some $R>0$,

$$
\min \left\{w(t): \overline{\mathfrak{e}}_{\Upsilon(t)} \in \overline{\mathfrak{f}}_{*}, \Upsilon(t) \neq i_{m}\right\}>\min \left\{w(t): \overline{\mathfrak{e}}_{\Upsilon(t)} \in \overline{\mathfrak{f}}_{*}\right\}+R / 2
$$

Then

$$
\begin{aligned}
& \operatorname{diam}\left(\operatorname{medqg}_{x_{1} x_{2}}^{D, R}\left(X_{\mathbf{q}}\right)\right) \\
& \quad \leq 2 \max _{\mathfrak{e} \in E} \omega\left(l^{\mathfrak{e}}, \operatorname{diam}\left\{i<\tau^{\mathfrak{e}}: w^{\mathfrak{e}}\left(\underline{c}^{\mathfrak{e}}(i)\right) \leq \inf w^{\mathfrak{e}}+R / 2\right\}\right)+R+4,
\end{aligned}
$$

Proof. - Recall that $X_{\mathbf{q}}$ is considered as a geodesic metric space, so that the quantity medqg $_{x_{1} x_{2}}^{D, R}$ makes sense. Assume that $x \in \operatorname{medqg}_{x_{1} x_{2}}^{D, R}\left(X_{\mathbf{q}}\right)$. We first argue that there exists an element of medqg ${ }_{x_{1} x_{2}}^{D, R}\left(X_{\mathbf{q}}\right) \cap V(\mathbf{q})$ at distance at most 1 from $x$. Indeed, if $x \notin V(\mathbf{q})$, then it must stand on an edge of the metric graph $\mathrm{X}_{\mathbf{q}}$ that links two vertices $y, z$. Thus any geodesic path from $x$ to $x_{1}$ or $x_{2}$ must pass through $y$ or $z$, and by symmetry we may assume $d_{\mathbf{q}}\left(x, x_{1}\right)=d_{\mathbf{q}}(x, y)+d_{\mathbf{q}}\left(y, x_{1}\right)$. If we also have $d_{\mathbf{q}}\left(x, x_{2}\right)=d_{\mathbf{q}}(x, y)+d_{\mathbf{q}}\left(y, x_{2}\right)$ then by subtracting $d_{\mathbf{q}}\left(y, x_{1}\right)-d_{\mathbf{q}}\left(y, x_{2}\right)=D$ and $y$ is the wanted vertex. Otherwise, it holds that $d_{\mathbf{q}}\left(x, x_{2}\right)=d_{\mathbf{q}}(x, z)+d_{\mathbf{q}}\left(z, x_{2}\right)$ so that

$$
D=d_{\mathbf{q}}\left(x, x_{1}\right)-d_{\mathbf{q}}\left(x, x_{2}\right)=d_{\mathbf{q}}\left(y, x_{1}\right)-d_{\mathbf{q}}\left(z, x_{2}\right)+\left(d_{\mathbf{q}}(x, y)-d_{\mathbf{q}}(x, z)\right) .
$$

As edges have length 1 , we have $d_{\mathbf{q}}(x, y)=1-d_{\mathbf{q}}(x, z)>0$, so the quantity in brackets is in $(-1,1)$, and the others are integers, so we must have $d_{\mathbf{q}}(x, y)=d_{\mathbf{q}}(x, z)=1 / 2$. But since $\left|d_{\mathbf{q}}\left(z, x_{2}\right)-d_{\mathbf{q}}\left(y, x_{2}\right)\right|=1$, this implies that $d_{\mathbf{q}}\left(y, x_{1}\right)-d_{\mathbf{q}}\left(y, x_{2}\right)$ has not the same parity as $D$. This contradicts the fact that $\mathbf{l}_{1}-\mathbf{l}_{2}$ takes values in $2 \mathbb{Z}$ established in Section 2.2. Thus, we may, and will, assume that $x \in V(\mathbf{q})$ up to increasing the diameter by 2 .

We next argue that there is a vertex $y \in V_{1} \cap V_{2}$ at $d_{\mathbf{q}}$-distance at most $R / 2$ from $x$. If $x$ itself is not such a vertex, then it is incident only to edges of $E_{i}(\mathbf{q}, \mathbf{x}, D)$ for some $i \in\{1,2\}$. Assume $i=1$ by symmetry and note that with the notations of Section 2.2, it holds that $\mathbf{l}_{1}(x)=\mathbf{l}_{2}(x)$. Take a chain of edges of $\mathbf{q}$ starting from $x$ and ending at $x_{2}$, along which the function $\mathbf{l}$ decreases, so that this chain is geodesic. It is easy to check that the first element $e$ of this chain which belongs to $E_{2}(\mathbf{q}, \mathbf{x}, D)$ is such that $y=e^{-}$is an element of $V_{1} \cap V_{2}$. Therefore, the chain from $x$ to $y$ can be extended as well to a geodesic from $x$ to $x_{1}$. Thus $D=d_{\mathbf{q}}\left(x, x_{1}\right)-d_{\mathbf{q}}\left(x, x_{2}\right)=d_{\mathbf{q}}\left(y, x_{1}\right)-d_{\mathbf{q}}\left(y, x_{2}\right)$ and $y$ is an element of $\operatorname{med}_{x_{1} x_{2}}^{D}\left(\mathrm{X}_{\mathbf{q}}\right) \cap V(\mathbf{q})$. Since $x \in \mathrm{qg}_{x_{1} x_{2}}^{R}$ and $y$ is on a geodesic from $x$ to $x_{1}$ and from $x$ to $x_{2}$, it is easily computed that $y$ is another element of $\mathrm{qg}_{x_{1} x_{2}}^{R}$ at distance at most $R / 2$ from $x$.

Therefore, we may, and will, restrict our attention to $x \in \operatorname{medqg}_{x_{1} x_{2}}^{D, R} \cap V_{1} \cap V_{2}$, up to increasing the diameter by $R+2$. Letting $(\mathbf{m},[\mathbf{l}])=\Psi_{g, 2}(\mathbf{q}, \mathbf{x}, D)$, the corresponding $x \in V(\mathbf{m})$ satisfies $d_{\mathbf{q}}\left(x, x_{1}\right)+d_{\mathbf{q}}\left(x, x_{2}\right)=2 \mathbf{l}(x)-\mathbf{l}\left(x_{1}\right)-\mathbf{l}\left(x_{2}\right) \leq d_{\mathbf{q}}\left(x_{1}, x_{2}\right)+R$, and together with (27) this yields

$$
\mathbf{l}(x) \leq \min _{V_{1} \cap V_{2}} \mathbf{l}+R / 2 .
$$


Now since $x$ is incident to $f_{*}$ and $\bar{f}_{*}$, we can find a time $t$ with $\mathbf{l}(x)=w(t)$, and $\overline{\mathfrak{e}}_{\Upsilon(t)}$ is incident to $\overline{\mathfrak{f}}_{*}$, and one sees after a moment's thought that the last inequality can be rewritten as

$$
\mathbf{l}(x)=w(t) \leq \min \left\{w(s): \overline{\mathfrak{e}}_{\Upsilon(s)} \in \overline{\mathfrak{f}}_{*}\right\}+R / 2 .
$$

These facts imply by hypothesis that $\Upsilon(t)=i_{m}$. Moreover, by Lemma 3, if $y$ is another vertex such as $x$, we have $d_{\mathbf{q}}(x, y) \leq 2 \omega\left(l^{e_{i_{m}}},\left|p-p^{\prime}\right|\right)+2$, where $\mathfrak{e}_{i_{m}}(p)^{-}=x$ and $\mathfrak{e}_{i_{m}}\left(p^{\prime}\right)^{-}=y$ with the notations of Section 3.3. Since $x$ and $y$ are incident to $f_{*}$ and $\bar{f}_{*}$, we have $w^{\mathfrak{e}}\left(\underline{\mathfrak{c}}_{p}^{\mathfrak{e}}\right)=\mathbf{l}(x)-\mathbf{l}\left(\mathfrak{e}_{i_{m}}(0)\right)$ and $w^{\mathfrak{e}}\left(\underline{c}_{p^{\prime}}^{\mathfrak{e}}\right)=\mathbf{l}(y)-\mathbf{l}\left(\mathfrak{e}_{i_{m}}(0)\right)$ which must be both $\leq \min w^{\mathfrak{e}}+R / 2$. Thus the result.

By Theorem 2, along the sequence $\left(a_{n}, n \geq 0\right)$, the spaces $a^{-1 / 4} \mathrm{X}_{\mathbf{q}}^{\mathrm{w}}$ under $\mathrm{Q}_{g}^{(\beta / a)}$ converge to $[X, d, \mu]$ in distribution, for some limiting $[X, d, \mu]$ with law $\mathscr{S}_{g}^{(\beta)}$. Together with Lemma 10 , this entails that the marked space $\left[X_{\mathbf{q}}, a^{-1 / 4} d_{\mathbf{q}},(x, y)\right]$ under $\mathrm{Q}_{g}^{(\beta / a)}(\mathrm{d} \mathbf{q}) \mu_{\mathbf{q}}^{\otimes 2}(x, y)$ converges to a space $[X, d,(x, y)]$ with law $\mathscr{S}_{g}^{(\beta)}(\mathrm{dX}) \mu^{\otimes 2}(\mathrm{~d}(x, y))$. On the other hand, the law of $\widetilde{\Psi}_{g, 2}(\mathbf{q},(x, y), D)$ under the law $\mathrm{Q}_{g, 2}^{(\beta / a)}$ defined by the formula

$\mathrm{Q}_{g}^{(\beta / a)}(\mathrm{d} \mathbf{q}) \mu_{\mathbf{q}}^{\otimes 2}(\mathrm{~d}(x, y)) \frac{\#_{\mathcal{D}(\mathbf{q},(x, y))}(\mathrm{d} D)}{|\mathcal{D}(\mathbf{q},(x, y))|}=\frac{\mathbf{Q}_{g, 2}\left(V_{\mathbf{q}}^{-2}|\mathcal{D}(\mathbf{q},(x, y))|^{-1} e^{-\beta V_{\mathbf{q}} / a} \mathrm{~d}(\mathbf{q},(x, y), D)\right)}{\mathbf{Q}_{g, 1}\left(V_{\mathbf{q}}^{-1} e^{-\beta V_{\mathbf{q}} / a}\right)}$

(recall (13)) equals

$\operatorname{LM}_{g, 2}^{(\beta / a)}\left(\mathrm{d}\left(\mathfrak{m}, \mathfrak{l}, t^{*}\right)\right):=\frac{\operatorname{LM}_{g, 2}\left(\left(\tau_{\mathfrak{l}} / 2+\chi(g)\right)^{-2}|\mathcal{D}(\mathbf{q},(x, y))|^{-1} e^{-\beta\left(\tau_{\mathfrak{l}} / 2+\chi(g)\right) / a} \mathrm{~d}\left(\mathfrak{m}, \mathfrak{l}, t^{*}\right)\right)}{\operatorname{LM}_{g, 1}\left(\left(\tau_{\mathfrak{l}} / 2+\chi(g)\right)^{-1} e^{-\beta\left(\tau_{\mathfrak{l}} / 2+\chi(g)\right) / a}\right)}$.

Here, we take the convention $|\mathcal{D}(\mathbf{q},(x, y))|^{-1}=0$ when $\mathcal{D}(\mathbf{q},(x, y))$ is empty. The random variable $|\mathcal{D}(\mathbf{q},(x, y))|=\left(d_{\mathbf{q}}(x, y)-1\right) \vee 0$ is a continuous function of $\left(\mathfrak{m}, \mathfrak{l}, t^{*}\right)$, since (27), (28) and (29) and the definition of $w$ imply

$$
d_{\mathbf{q}}(x, y)=2 \min _{t: \overline{\mathfrak{e}}_{\Upsilon(t)} \in \overline{\mathfrak{f}}_{*}} w(t)-\min l-\min l^{\prime}-\sum_{i=1}^{i_{0}-1} \widehat{l}^{\mathfrak{e}_{i}}-2 .
$$

We let $\mathfrak{d}$ denote the latter quantity with the -2 omitted, which can be associated with any element of $\mathbf{C}_{g, 2}$ (not necessarily in $\mathbf{C}_{g, 2}^{\mathrm{map}}$ ).

Lemma 22. - The law of $\xi^{a}\left(\mathfrak{m}, \mathfrak{l}, t^{*}\right)$ under $\mathrm{LM}_{g, 2}^{(\beta / a)}$ converges towards the law $\mathrm{CLM}_{g, 2}^{(\beta)}$ defined by

$$
\operatorname{CLM}_{g, 2}^{(\beta)}\left(\mathrm{d}\left(\mathfrak{m}, \mathfrak{l}, t^{*}\right)\right)=3 \frac{\operatorname{CLM}_{g, 2}\left(\tau_{\mathfrak{l}}^{-2} \mathfrak{d}^{-1} e^{-\beta \tau_{\mathfrak{l}}} \mathrm{d}\left(\mathfrak{m}, \mathfrak{l}, t^{*}\right)\right)}{\operatorname{CLM}_{g, 1}\left(\tau_{\mathfrak{l}}^{-1} e^{-\beta \tau_{\mathfrak{l}}}\right)}
$$

Proof. - Let $H$ be bounded continuous on $\mathbf{C}_{g, 2}$. Let $h_{\varepsilon}$ be 0 on $[0, \varepsilon / 2]$, equal to 1 on $[\varepsilon, \infty)$ and interpolate linearly in-between. Let $H_{\varepsilon}\left(\mathfrak{m}, \mathfrak{l}, t^{*}\right)=H\left(\mathfrak{m}, \mathfrak{l}, t^{*}\right) h_{\varepsilon}(\mathfrak{d}) h_{\varepsilon}\left(\tau_{\mathfrak{l}}\right)$. Then a straightforward use of Theorem 5 entails that $\operatorname{LM}_{g, 2}^{(\beta / a)}\left(H_{\varepsilon} \circ \xi^{a}\right)$ converges to $\operatorname{CLM}_{g, 2}^{(\beta)}\left(H_{\varepsilon}\right)$. When $H$ is nonnegative, this quantity converges as $\varepsilon \rightarrow 0$ to $\operatorname{CLM}_{g, 2}^{(\beta)}(H)$, and in particular $\mathrm{CLM}_{g, 2}^{(\beta)}$ is a sub-probability measure. Thus, by dominated convergence, this last convergence holds for $H$ not necessarily non-negative.

On the other hand, we know that $\left(\tau_{\mathfrak{l}} / 2+\chi(g), \mathfrak{d}\right)$ has same law under $\operatorname{LM}_{g, 2}^{(\beta / a)}$ as $\left(V_{\mathbf{q}}, d_{\mathbf{q}}(x, y)+2\right)$ under $\mathrm{Q}_{g}^{(\beta / a)}(\mathrm{d} \mathbf{q}) \mu_{\mathbf{q}}^{\otimes 2}(\mathrm{~d}(x, y))$. So $\left(a^{-1}\left(\tau_{\mathfrak{l}} / 2+\chi(g)\right), a^{-1 / 4} \mathfrak{d}\right)$ under $\mathrm{LM}_{g, 2}^{(\beta / a)}$ converges in distribution to $(\mathcal{V}, d(x, y))$ under $\mathscr{S}_{g}^{(\beta)}(\mathrm{d}(\mathrm{X}, \mathcal{V})) \mu^{\otimes 2}(\mathrm{~d}(x, y))$ (here we 
used Proposition 10 for the convergence of the distance). Since we know that $\mu$ is a.s. diffuse, it holds that $d(x, y)>0$ a.s. under the latter measure, entailing the tightness of the variables $a^{-1 / 4} \mathfrak{d}$ under $\operatorname{LM}_{g, 2}^{(\beta / a)}$ in $(0, \infty)$. Since $a^{-1} V_{\mathbf{q}}=a^{-1}\left(\tau_{\mathfrak{l}} / 2+\chi(g)\right)$ under $\operatorname{LM}_{g, 2}^{(\beta / a)}$ are tight in $(0, \infty)$ as well, this entails that for some positive $\eta(\varepsilon) \rightarrow 0$ as $\varepsilon \rightarrow 0$,

$$
\left|\operatorname{LM}_{g, 2}^{(\beta / a)}\left(H \circ \xi^{a}\right)-\operatorname{LM}_{g, 2}^{(\beta / a)}\left(H_{\varepsilon} \circ \xi^{a}\right)\right| \leq\|H\|_{\infty} \eta(\varepsilon) .
$$

By first taking $\varepsilon$ small enough and then letting $a \rightarrow \infty$, we conclude that $\operatorname{LM}_{g, 2}^{(\beta / a)}\left(H \circ \xi^{a}\right) \rightarrow \operatorname{CLM}_{g, 2}^{(\beta)}(H)$, as wanted.

As in the beginning of Section 7.2.1, we make the assumption that we are working with a probability space $(\Omega, \mathcal{F}, P)$ under which are defined random variables $\left(\mathbf{q}_{a},\left(x_{a}, y_{a}\right), D_{a}\right)$ with respective laws $\mathbf{Q}_{g}^{(\beta / a)}(\mathrm{d} \mathbf{q}) \mu_{\mathbf{q}}^{\otimes 2}(\mathrm{~d}(x, y))|\mathcal{D}(\mathbf{q},(x, y))|^{-1} \#_{\mathcal{D}(\mathbf{q},(x, y))}(\mathrm{d} D)$, such that, along $a_{n}, n \geq 1$,

1. the marked spaces $\left[X_{a}, a^{-1 / 4} d_{a},\left(x_{a}, y_{a}\right)\right]$ converge a.s. in $\mathrm{PM}_{2}^{*}$ to $[X, d,(x, y)]$ with law $\pi_{*}^{1} \mathscr{S}_{g}^{(\beta)}(\mathrm{dX}) \mathrm{m}_{2}(\mathrm{X}, \mathrm{d}(x, y))$, where $\pi^{1}: \mathrm{PM}_{\mathrm{w}} \times(0, \infty) \rightarrow \mathrm{PM}_{\mathrm{w}}$ is the first projection,

2. $a^{-1 / 4} D_{a} \rightarrow D$ a.s., the latter having uniform law on $(-d(x, y), d(x, y))$ given $d(x, y)$, and

3. letting $\Psi_{g, 2}\left(\mathbf{q}_{a},\left(x_{a}, y_{a}\right), D_{a}\right)=\left(\mathbf{m}_{a},\left[\mathbf{l}_{a}\right]\right)$ and $\widetilde{\Psi}_{g, 2}\left(\mathbf{q}_{a},\left(x_{a}, y_{a}\right), D_{a}\right)=\left(\mathfrak{m}_{a}, \mathfrak{l}_{a}, t_{a}^{*}\right)$, we have a.s. $\xi^{a}\left(\mathfrak{m}_{a}, \mathfrak{l}_{a}, t_{a}^{*}\right) \rightarrow\left(\mathfrak{m}, \mathfrak{l}, t^{*}\right)$ where the limit has law $\operatorname{CLM}_{g, 2}^{(\beta)}$.

LEMма 23. - The following events are of full measure under $\mathrm{CLM}_{g, 2}$ :

- The quantity $\inf \left\{w(t): \overline{\mathfrak{e}}_{\Upsilon(t)} \in \overline{\mathfrak{f}}_{*}\right\}$ is attained for a unique time $t$, which is not a jump time of $\Upsilon(t)$ and is distinct from 0 and $\tau(w)$.

- The quantities diam $\left\{0 \leq s \leq \tau^{\mathfrak{e}}: w^{\mathfrak{e}}\left(\underline{\underline{e}}^{\mathfrak{e}}(s)\right) \leq \inf w^{\mathfrak{e}}+\delta\right\}$ for $\mathfrak{e} \in E$ decrease to 0 as $\delta \downarrow 0$.

Proof. - Under the measure $\mathrm{CLM}_{g, 2}$ and conditionally on $\mathfrak{m}, \mathbf{r},\left(\widehat{w}^{\mathfrak{e}}, \mathfrak{e} \in E(\mathfrak{m})\right)$, the processes $w^{\mathfrak{e}}$ are independent Brownian bridges. Since the minimum value attained by a Brownian bridge has a diffuse law, and is attained a.s. once by the path at a time which is neither the starting nor the ending time of the bridge, the first point is clear.

Now conditionally on $\mathfrak{m}, \mathbf{r},\left(w^{\mathfrak{e}}, \mathfrak{e} \in E(\mathfrak{m})\right)$, the paths $\left(c^{\mathfrak{e}}, z^{\mathfrak{e}}\right)$ are independent Brownian snakes under $\mathrm{CLM}_{g, 2}$. If $s_{m}^{\mathfrak{e}}$ is the (unique) time at which $w^{\mathfrak{e}}$ attains its minimum, then it follows from standard properties of Brownian motion that $\underline{c}^{\mathfrak{e}}$ (the infimum process of $c^{\mathfrak{e}}$ ) attains the value $s_{m}$ at a unique time $t_{m}$ with probability 1 . This means that $\underline{c}_{t}^{\mathfrak{e}}<\underline{c}_{t_{m}}^{\mathfrak{e}}<\underline{t}_{t^{\prime}}^{\mathfrak{e}}$ for every $t<t_{m}<t^{\prime}$, and by continuity the two bounding terms converge towards the middle one as $t, t^{\prime} \rightarrow t_{m}$. Combining this with the continuity of $w^{\mathfrak{e}}$ and the fact that the value inf $w^{\mathfrak{e}}$ is attained only at $s_{m}^{\mathfrak{e}}$, we obtain the result.

We can now finish the proof of Theorem 3. By the first point in Lemma 23 and the a.s. convergence of $\xi^{a}\left(\mathfrak{m}_{a}, \mathfrak{l}_{a}, t_{a}^{*}\right)$ to $\left(\mathfrak{m}, \mathfrak{l}, t^{*}\right)$, we conclude that $\left(\mathfrak{m}_{a}, \mathfrak{l}_{a}, t_{a}^{*}\right)$ satisfies the hypotheses of Lemma 21 for $R$ in the form $\delta a^{1 / 4}$ with $\delta$ small enough, and every large enough $a$, almost-surely. From the latter lemma and to be able to apply Lemma 11 to the spaces $\left[X_{a}, a^{-1 / 4} d_{a},\left(x_{a}, y_{a}\right)\right]$ and the delays $D_{a}$, it suffices to notice that for every $\eta>0$, there exists $\delta \in(0, \eta)$ such that for every $a, \mathfrak{e}$,

$$
\operatorname{diam}\left(\left\{i<\tau_{a}^{\mathfrak{e}}: w_{a}^{\mathfrak{e}}\left(\underline{c}_{a}^{\mathfrak{e}}(i)\right) \leq \inf w_{a}^{\mathfrak{e}}+a^{1 / 4} \delta / 2\right\}\right) \leq 2 a \eta,
$$


as follows from the second point of Lemma 23 and the a.s. uniform convergence of the processes $\phi^{a}\left(w_{a}^{\mathfrak{e}}\right), \vartheta^{a}\left(c_{a}^{\mathfrak{e}}\right)$ towards $w^{\mathfrak{e}}, c^{\mathfrak{e}}$. Then, taking $\varepsilon>0$ and choosing $\eta$ in turn so that $2 \varsigma \omega\left(\psi^{a}\left(l_{a}^{\mathfrak{e}}\right), \eta\right)+\eta<\varepsilon$ for every $a, \mathfrak{e}$, which is possible by the uniform convergence of $\psi^{a}\left(l_{a}^{\mathfrak{e}}\right)$ to $l^{\mathfrak{e}}$, Lemma 21 implies that

$$
\limsup _{a \rightarrow \infty} \operatorname{diam}\left(\operatorname{medqg}_{x y}^{a^{-1 / 4} D_{a}, \delta}\left(a^{-1 / 4} \mathrm{X}_{a}\right)\right) \leq \varepsilon
$$

Therefore, Lemma 11 implies that a.s. under

$$
\mathscr{S}_{g}^{(\beta)}(\mathrm{d}(\mathrm{X}, \mathcal{V})) \mu^{\otimes 2}(\mathrm{~d}(x, y)) \mathrm{d} D \mathbb{1}_{[-d(x, y), d(x, y)]}(D) / 2 d(x, y),
$$

all geodesic paths from $x$ to $y$ pass through a unique $D$-median point. This shows that a.s. under the measure $\mathscr{S}_{g}^{(\beta)}(\mathrm{d}(\mathrm{X}, \mathcal{V})) \mu^{\otimes 2}(\mathrm{~d}(x, y))$, the geodesics from $x$ to $y$ all pass through a unique $D$-median point, for Lebesgue-almost every $D$. Since $D$-median points form a continuous parametrization in $D \in[-d(x, y), d(x, y)]$ of geodesics from $x$ to $y$, this implies that a.s. the geodesic between $\mu$-almost all $x$ and $y$ is unique, hence the result.

\section{REFERENCES}

[1] D. Aldous, The continuum random tree. I, Ann. Probab. 19 (1991), 1-28.

[2] J. Ambjørn, B. Durhuus, T. Jonsson, Quantum geometry. A statistical field theory approach, Cambridge Monographs on Mathematical Physics, Cambridge Univ. Press, 1997.

[3] O. Angel, Growth and percolation on the uniform infinite planar triangulation, Geom. Funct. Anal. 13 (2003), 935-974.

[4] E. A. Bender, E. R. CANField, The asymptotic number of rooted maps on a surface, J. Combin. Theory 43 (1986), 244-257.

[5] P. Billingsley, Convergence of probability measures, second ed., Probability and Statistics, John Wiley \& Sons Inc., 1999.

[6] N. Bourbaki, Éléments de mathématique, Topologie générale, chap. 1 à 4, Hermann, 1971.

[7] J. Bouttier, P. Di Francesco, E. Guitter, Planar maps as labeled mobiles, Electron. J. Combin. 11 (2004), Research Paper 69 (electronic).

[8] J. Bouttier, E. Guitter, Statistics in geodesics in large quadrangulations, J. Phys. 41 (2008), 145001, 30.

[9] D. Burago, Y. Burago, S. Ivanov, A course in metric geometry, Graduate Studies in Math. 33, Amer. Math. Soc., 2001.

[10] G. Chapuy, M. Marcus, G. Schaeffer, A bijection for rooted maps on orientable surfaces, preprint arXiv:0712.3649, 2007.

[11] P. Chassaing, G. Schaeffer, Random planar lattices and integrated superBrownian excursion, Probab. Theory Related Fields 128 (2004), 161-212.

[12] R. M. Dudley, Real analysis and probability, Cambridge Studies in Advanced Math. 74, Cambridge Univ. Press, 2002.

[13] T. Duquesne, A limit theorem for the contour process of conditioned Galton-Watson trees, Ann. Probab. 31 (2003), 996-1027. 
[14] T. Duquesne, J.-F. Le Gall, Random trees, Lévy processes and spatial branching processes, Astérisque 281 (2002).

[15] T. Duquesne, J.-F. Le Gall, Probabilistic and fractal aspects of Lévy trees, Probab. Theory Related Fields 131 (2005), 553-603.

[16] S. N. Evans, J. Pitman, A. Winter, Rayleigh processes, real trees, and root growth with re-grafting, Probab. Theory Related Fields 134 (2006), 81-126.

[17] S. N. Evans, A. Winter, Subtree prune and regraft: a reversible real tree-valued Markov process, Ann. Probab. 34 (2006), 918-961.

[18] P. Flajolet, R. Sedgewick, Analytic combinatorics, Cambridge Univ. Press, 2009.

[19] K. Fukaya, Collapsing of Riemannian manifolds and eigenvalues of Laplace operator, Invent. Math. 87 (1987), 517-547.

[20] Z. GAO, A pattern for the asymptotic number of rooted maps on surfaces, J. Combin. Theory 64 (1993), 246-264.

[21] A. Greven, P. Pfaffelhuber, A. Winter, Convergence in distribution of random metric measure spaces ( $\Lambda$-coalescent measure trees), preprint, 2006.

[22] M. Gromov, Metric structures for Riemannian and non-Riemannian spaces, Progress in Math. 152, Birkhäuser, 1999.

[23] S. Janson, J.-F. Marckert, Convergence of discrete snakes, J. Theoret. Probab. 18 (2005), 615-647.

[24] S. K. Lando, A. K. Zvonkin, Graphs on surfaces and their applications, Encyclopaedia of Math. Sciences 141, Springer, 2004.

[25] J.-F. LE GALL, The uniform random tree in a Brownian excursion, Probab. Theory Related Fields 96 (1993), 369-383.

[26] J.-F. Le GALL, The topological structure of scaling limits of large planar maps, Invent. Math. 169 (2007), 621-670.

[27] J.-F. Le GALL, Geodesics in large planar maps and in the Brownian map, preprint arXiv:0804.3012, 2008.

[28] J.-F. Le Gall, F. Paulin, Scaling limits of bipartite planar maps are homeomorphic to the 2-sphere, Geom. Funct. Anal. 18 (2008), 893-918.

[29] T. LÉvy, Yang-Mills measure on compact surfaces, Mem. Amer. Math. Soc. 166 (2003).

[30] J.-F. Marckert, G. Miermont, Invariance principles for random bipartite planar maps, Ann. Probab. 35 (2007), 1642-1705.

[31] J.-F. Marckert, A. Mok Kadem, States spaces of the snake and its tour-convergence of the discrete snake, J. Theoret. Probab. 16 (2003), 1015-1046.

[32] J.-F. Marckert, A. MokKadem, Limit of normalized quadrangulations: the Brownian map, Ann. Probab. 34 (2006), 2144-2202.

[33] M. Marcus, G. SchaefFer, Une bijection simple pour les cartes orientables, preprint

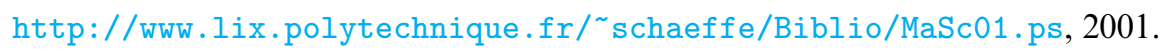

[34] G. Miermont, An invariance principle for random planar maps, in Fourth Colloquium on Mathematics and Computer Sciences CMCS'06, Discrete Math. Theor. Comput. Sci. Proc., 2006, 39-58 (electronic).

[35] G. Miermont, Invariance principles for spatial multitype Galton-Watson trees, Ann. Inst. Henri Poincaré Probab. Stat. 44 (2008), 1128-1161.

$4^{\mathrm{e}}$ SÉRIE - TOME $42-2009-\mathrm{N}^{\mathrm{o}} 5$ 
[36] G. Miermont, M. Weill, Radius and profile of random planar maps with faces of arbitrary degrees, Electron. J. Probab. 13 (2008), 79-106.

[37] A. Oкоunkov, Random matrices and random permutations, Int. Math. Res. Not. 2000 (2000), 1043-1095.

[38] A. Okounkov, R. Pandharipande, Gromov-Witten theory, Hurwitz numbers, and matrix models, in Algebraic geometry-Seattle 2005. Part 1, Proc. Sympos. Pure Math. 80, Amer. Math. Soc., 2009, 325-414.

[39] V. V. Petrov, Sums of independent random variables, Springer, 1975.

[40] J. Pitman, Combinatorial stochastic processes, Lecture Notes in Math. 1875, Springer, 2006.

[41] D. Revuz, M. Yor, Continuous martingales and Brownian motion, third ed., Grund. Math. Wiss. 293, Springer, 1999.

[42] G. Schaeffer, Conjugaison d'arbres et cartes combinatoires aléatoires, Thèse de doctorat, Université Bordeaux I, 1998.

[43] S. Sheffield, Gaussian free fields for mathematicians, Probab. Theory Related Fields 139 (2007), 521-541.

[44] C. Villani, Optimal transport, Grund. Math. Wiss. 338, Springer, 2009.

[45] M. WeILL, Asymptotics for rooted bipartite planar maps and scaling limits of two-type spatial trees, Electron. J. Probab. 12 (2007), 887-925.

(Manuscrit reçu le 11 février 2008;

accepté, après révision, le 27 avril 2009.)

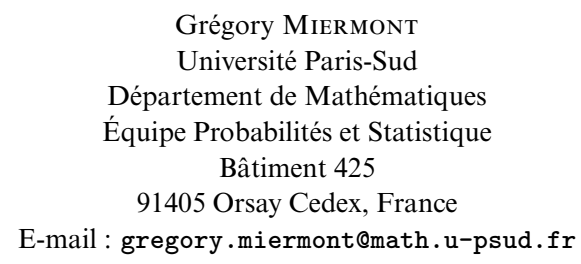

